\title{
Public health genomics of infectious diseases: insights gained from chlamydia trachomatis infertility diagnostics
}

Citation for published version (APA):

Malogajski, J. (2014). Public health genomics of infectious diseases: insights gained from chlamydia trachomatis infertility diagnostics. [Doctoral Thesis, Maastricht University]. Maastricht University. https://doi.org/10.26481/dis.20141216jm

Document status and date:

Published: 01/01/2014

DOI:

10.26481/dis.20141216jm

Document Version:

Publisher's PDF, also known as Version of record

Please check the document version of this publication:

- A submitted manuscript is the version of the article upon submission and before peer-review. There can be important differences between the submitted version and the official published version of record.

People interested in the research are advised to contact the author for the final version of the publication, or visit the DOI to the publisher's website.

- The final author version and the galley proof are versions of the publication after peer review.

- The final published version features the final layout of the paper including the volume, issue and page numbers.

Link to publication

\footnotetext{
General rights rights.

- You may freely distribute the URL identifying the publication in the public portal. please follow below link for the End User Agreement:

www.umlib.nl/taverne-license

Take down policy

If you believe that this document breaches copyright please contact us at:

repository@maastrichtuniversity.nl

providing details and we will investigate your claim.
}

Copyright and moral rights for the publications made accessible in the public portal are retained by the authors and/or other copyright owners and it is a condition of accessing publications that users recognise and abide by the legal requirements associated with these

- Users may download and print one copy of any publication from the public portal for the purpose of private study or research.

- You may not further distribute the material or use it for any profit-making activity or commercial gain

If the publication is distributed under the terms of Article $25 \mathrm{fa}$ of the Dutch Copyright Act, indicated by the "Taverne" license above, 
Public Health Genomics of Infectious

Diseases: Insights gained from

Chlamydia trachomatis-induced infertility

diagnostics 
(c) Copyright Jelena Malogajski, Maastricht 2014 Cover design Tatjana Kuburović

Layout Bruka Notes

Printed by Ipsakamp Drukkers

ISBN: 978-94-6259-462-3 


\title{
Public Health Genomics of Infectious Diseases: Insights gained from Chlamydia trachomatis-induced infertility diagnostics
}

\author{
Dissertation \\ To obtain the degree of Doctor at the Maastricht University, \\ on the authority of the Rector Magnificus, Prof. L. L. G. Soete, \\ in accordance with the decision on the Board of Deans, \\ to be defended in public on
}

Tuesday, $16^{\text {th }}$ December 2014 at 12.00 hrs.

by

Jelena Malogajski 
SUPERVISORS

Prof. dr. Servaas A. Morré

Prof. dr. Angela Brand

CO-SUPERVISOR

Dr. Elena Ambrosino

ASSESSMENT COMMITTEE

Prof. dr. Hans Evers, Chair

Dr. Peter Schröder-Bäck

\section{Prof. dr. Paul Savelkoul}

VU University Medical Center, Amsterdam

Dr. Joke Spaargaren

West-Friesgasthuis, Zaans Medical Center at Atal-medial 
CONTENTS

General Introduction $\mid 7$

Chapter 1 Translational Potential into Health Care of Basic Genomic and Genetic Findings for Human Immunodeficiency Virus, Chlamydia trachomatis, and Human papillomavirus $\mid 27$

Chapter 2 Biobanking and translation of human genetics and genomics for infectious diseases $\mid 53$

Chapter 3 Chlamydia trachomatis infections and subfertility: opportunities to translate host pathogen genomic data into Public Health $\mid 77$

Chapter 4 Diagnosis of Chlamydia trachomatis induced tubal pathology in infertile women through genomic diagnostics - exploration of health needs $\mid 105$

Chapter 5 The attitudes of the Dutch gynaecologists towards the addition of genetic testing in the diagnosis of tubal factor infertility (TFI) $\mid 125$

Chapter 6 General discussion $\mid 141$

Chapter 7 Valorisation addendum | 155

Summary $\mid 161$

Samenvatting $\mid 167$

Acknowledgment | 173

Curriculum vitae 175

Appendix 177 

GENERAL INTRODUCTION 


\section{INTRODUCTION}

\section{Content}

Translation of genomic discoveries

Genomic Information and Infectious diseases

Chlamydia trachomatis

Immunogenetics of Chlamydia

Chlamydia trachomatis host genetic factors

Chlamydia induced infertility

Aim of the thesis

Outline of the thesis

\section{Translation of genomic discoveries}

The Human Genome Project marked the beginning of the genome era, and was expected to revolutionize the field of biomedical science and unlock the secrets of the role of genetic variants in human disease. Indeed, "the magnitude, scope and pace of discovery in genetics and genomics research are at unprecedented levels and continue to increase exponentially" [1]. It also raised the bar on the expectations for the impact these discoveries will have on advances in clinical application of genomic knowledge. Today, many gene-disease associations are proven and genetic/genomic testing has acquired a role in guiding clinical decision making and in treatment of disease.

However, there is a prevailing sense that the process of integration and translation of genomic discoveries in clinical practice is hindered by the lack of information on the clinical validity and utility of these discoveries on one hand, and the lack of knowledge on the side of the health providers about the proven benefits of the discoveries [2].

Recently it has been estimated that more than 15,000 tests exist for more than 2,800 genes [3]. Performing horizon scanning for new genomic tests on the Internet, Gwinn et al. detected approximately 2 to 3 new genomic tests coming out per week [4].

Large number of genomic tests appear at the market every year, yet only few of them are sufficiently supported by evidence to justify their clinical use [5]. This chasm between the use of these tests and improved health outcomes has been 
described as the "lost in translation" gap [6]. Khoury [7] offered the solution the problem of translation gap of genomic discoveries through approach called Public Health approach to genetics (Figure 1).

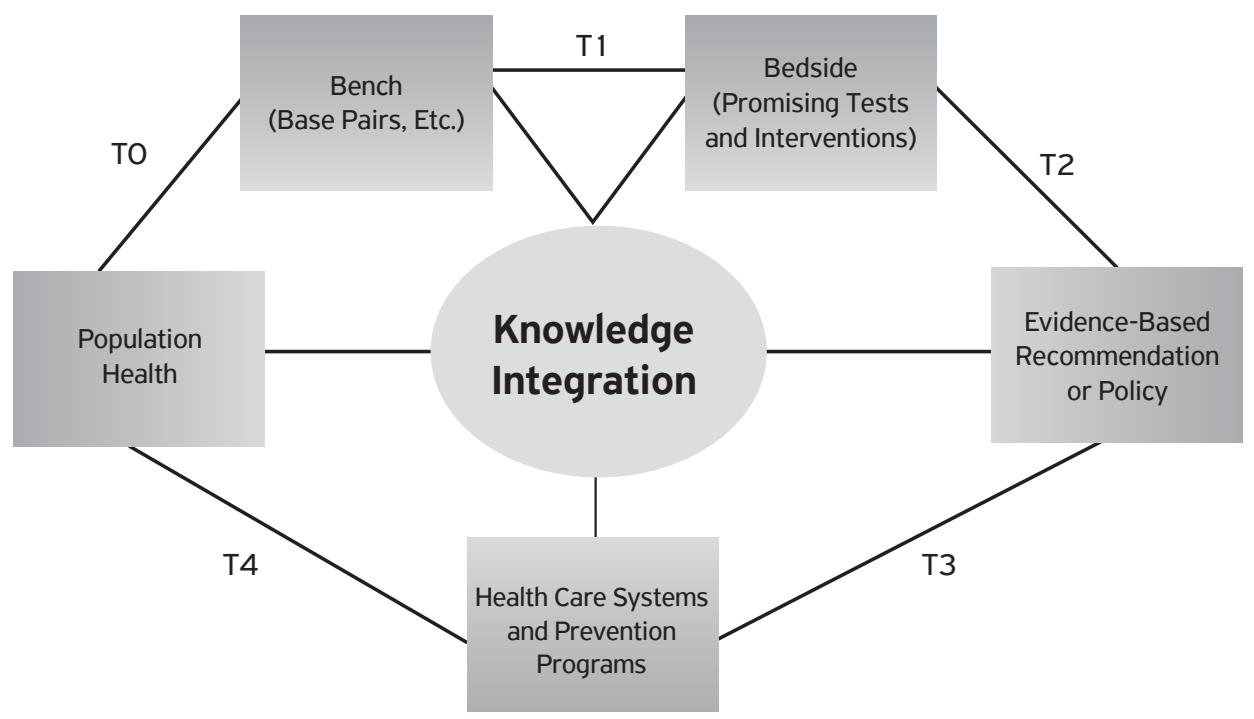

Figure 1. Public Health Genomics model of the translational research (TO-T4) needed to convert discoveries into better health (source: Khoury, Genome-Based Diagnostics: Clarifying Pathways to Clinical Use: Workshop Summary, 2012) [7]

Phase TO represents discovery research, Phases T1 and T2 include observational studies and clinical trials, starting with gene discovery, progressing through the development of the health application and ending with the development of evidence based guidelines. Phase T3 of the translation research aims at ensuring the success of the evidence based health intervention in practice. It involves dissemination of the knowledge about the intervention and activities aiming at successful implementation of the intervention. Phase T4 is concerned with the impact that the new application/intervention will have on the clinical and public health outcomes [6].

The analysis of grants attributed to these different phases of translational research in the field of cancer genomics showed that less than $2 \%$ of all grants are focusing on later stages of translation process, including the studies of clinical utility. This analysis identified therefore another current problem in the 
process of translation of genomic discoveries into clinical practice, the lack of resources in translational research [8].

Despite the existing problems, there are successful examples of genomic information being used in prevention and everyday clinical practice, improving health outcomes and supporting some of expectations bestowed upon genomic discoveries. The most commonly described are BRCA1/BRCA2 test and pharmacogenetic tests. Mutation in the BRCA1 or BRCA2 genes (genes responsible for production of tumor suppressor proteins, with a role in repairing damaged DNA) significantly increases risk of developing breast and/or ovarian cancer in women who inherited them. Genetic tests can check for BRCA 1 and BRCA2 mutations in people with a family history of cancer, suggesting the presence of a harmful mutation in one of these genes. Women identified with having mutations in one or both BRCA genes are candidates for more frequent breast cancer screening, risk reducing surgery or chemoprevention, as ways of managing their cancer risk [9]. A different example of successful use of genomic information in practice are pharmacogenetic tests aiming at improving the safety and efficacy of drug treatment, as well as guiding appropriate dosing of medicines.

Oral anticoagulant Warfarin is a well-known example of a drug made safer and more effective by determining the dosage based on the genetic makeup of a patient. Polymorphisms in the gene encoding for the enzyme cytochrome P-450 2C9 (CYP2C9) are associated with variability in sensitivity to warfarin. Patients with certain genetic variants of CYP2C9 are at risk for over-anticoagulation and serious bleeding, therefore require a lower dose of warfarin and a longer time to reach a stable dose [10].

In both mentioned examples (BRCA1/BRCA2 test and pharmacogenomics tests) the useful application of a genetic test is linked to an intervention that improves health outcomes [1 11 .

The trends in using genome based information with the aim to improve health outcomes of population have been changing over time. Initially, most of the tests were developed to diagnose or confirm classic Mendelian disorders [12]. These diseases are characterized by traits inherited in a monogenic fashion, the changes in a single gene are responsible for almost all the disease manifestations, individual alleles are inherited in families according to Mendel's law [13]. Technological advances allowing the new generation of genome wide 
association studies contributed to the attention gradually moving towards common complex diseases, such as diabetes, cancer and heart diseases, whose pathophysiology is characterized by an interplay of genetic and environmental factors [14].

Recent review of the new genomic tests, categorized by diseases or condition, showed that $65 \%$ of these tests are designed for oncology (Table 1) [4].

Table 1. Distribution of new genomic tests by disease or condition (source: Gwinn et al., 2011) [4]

\begin{tabular}{|c|c|c|}
\hline & Number & Percent \\
\hline Cancer & 122 & 65 \\
\hline Colorectal & 25 & \\
\hline Lung & 21 & \\
\hline Breast & 17 & \\
\hline Prostate & 13 & \\
\hline Ovary & 6 & \\
\hline Leukemia/lymphoma & 6 & \\
\hline Skin & 6 & \\
\hline Bladder & 5 & \\
\hline Mesothelioma & 3 & \\
\hline Pancreas & 3 & \\
\hline Gastric & 2 & \\
\hline Other & 4 & \\
\hline Multiple sites & 3 & \\
\hline Not specified & 8 & \\
\hline Cardiovascular & 19 & 10 \\
\hline Conorary heart disease & 7 & \\
\hline Clotting disorders & 6 & \\
\hline Cardiac conduction disorders & 4 & \\
\hline Hypertension & 1 & \\
\hline Not specified & 1 & \\
\hline Gastrointestinal disorders & 5 & 3 \\
\hline Neurodegenerative disorders & 4 & 2 \\
\hline Developmental disorders/birth defects & 4 & 2 \\
\hline Age related macular degeneration & 2 & 2 \\
\hline Autoimmune disorders & 2 & 1 \\
\hline Diabetes/metabolic syndrome & 2 & 1 \\
\hline Psychiatric disorders & 2 & 1 \\
\hline Hereditary disorders & 11 & 6 \\
\hline Other & 13 & \\
\hline Total & 188 & \\
\hline
\end{tabular}


Genotyping of somatic mutations in cancer cells, for the purpose of guiding medical decisions in cancer diagnosis and therapy, for selected cancers, is leading the way among the genomic technologies which reached clinical practice. Somatic mutations found in tumors can influence the response to therapy and/or disease outcome. For example, lung cancer, melanoma and Myeloproliferative disorders with mutations in the genes EGFR, BRAF and JAK 2 respectively have a tendency of being sensitive to tyrosine kinase inhibitors (TKI) $[15,16]$. Furthermore, in order to identify individuals genetically at risk of developing cancer, relatives of patients with germline mutations cancers are undergoing screening (e.g. Lynch syndrome and aforementioned BRCA 1,2). Pharmacogenetic tests, as important tools in personalized approach to medication therapy follow. Identifying patients genetically unable to use certain drugs and guiding the adequate dosing of other medications improves drug selection in patients and increases efficacy and safety of medications. Finally, whole genome and whole exome sequencing is used in diagnosis of rare unknown diseases [17]. One of the first successful examples whole exome sequencing was used to reach a clinical diagnosis and guide the treatment of a child with a life-threatening but previously undefined form of inflammatory bowel disease in whom standard treatments were ineffective. Next generation sequencing of the patient's exome, together with confirmatory studies, revealed $\mathrm{X}$-linked inhibitor of apoptosis (XIAP) deficiency [18].

\section{Genomic Information and Infectious diseases}

The understanding of the importance of genomic information in diagnosis and treatment of infectious diseases has been staidly increasing in recent years [19].

Traditionally infectious diseases have been observed as a result of the interplay of microorganism properties, transmission mechanisms, and the host. Scientific developments in genomics changed the way we think about infectious diseases. The recent evidence of genetic factors in the pathogenesis of infectious diseases transformed our view of such diseases by recognizing the role of host genetic determinants that modulate immune response. Progress in genomics led to the characterization of molecular biomarkers and pathways as targets for diagnosis or intervention [20]. Furthermore, this new understanding of 
infectious diseases explains why some individuals are just more resistant/ susceptible to an infection. It also explains the differences in clinical course of the infection by, among other factors, host genetic makeup. A great amount of effort and resources has been directed to obtaining knowledge about host genetic components of infectious diseases and to confirm associations with an aim of developing genomic applications and translating them into everyday clinical practice and prevention (Table 2).

Table 2. Examples of replicated genetic associations with human infectious diseases (adopted from Hill, 2006) [20]

\begin{tabular}{|l|l|}
\hline DISEASE & GENETIC LOCUS \\
\hline Malaria & $\begin{array}{l}\text { alpha-globin, beta-globin, G6PD, } \\
\text { SLC4A1, MAL/TIRAP, DARC }\end{array}$ \\
\hline Tuberculosis & $\begin{array}{l}\text { HLA-DR, INF-Y, SLC11 A1, } \\
\text { VDR, MAL/TIRAP, CCL2 }\end{array}$ \\
\hline HIV/AIDS & HLA-B, CCR5, CCR2 \\
\hline
\end{tabular}

When it comes to public health strategies regarding infectious diseases conventional approach can be summarized in 3 words: prevention, treatment, and control. Revolutionary breakthrough in genome research facilitated improvements in public health interventions, widening its scope of interest and activities. Traditional public health efforts aiming at preventing, controlling and treating infectious diseases [21] expanded to include responsible and effective translation of genome-based knowledge and technologies into public policy and health services for the benefit of population health through the establishment of the field of Public Health Genomics [22].

Infectious diseases represent a major health threat worldwide and a significant part of the burden of disease in developing countries [23]. They are still the leading death cause worldwide. Despite the epidemiological transition characterized by decline in morbidity and mortality related to infectious diseases and shift from infectious diseases to chronic complex diseases [15], some infectious diseases are on the rise in the developed countries.

In Europe, data obtained from the Sexually Transmitted Infections (STI) surveillance reports of the EU countries are showing stable or increasing trends 
in recent years, in most of the countries [24]. Reports show that STIs represent a major public health problem in Europe. Indeed, European surveillance data show that Chlamydia trachomatis genital infection has been on the rise since the mid-1990s and, in fact, is at the moment the most commonly diagnosed bacterial STI [25].

\section{Chlamydia trachomatis}

Chlamydia trachomatis is a sexually transmitted intracellular pathogen causing more cases of Sexually Transmitted Infections than any other bacteria. [26]. The WHO estimated the global incidence of Chlamydia to be 105.7 million cases in 2008, an increase of 4.1 percent compared to 101.5 million cases in 2005. In addition to really high incidence what makes Chlamydia infection a significant public health problem, despite the fact that the microorganism can be successfully cleared by antibiotics, is that $80-90 \%$ of infections in women and up to $50 \%$ in men are asymptomatic $[27,28]$. As a result infection often remains untreated, and its consequences can be particularly severe in women. In untreated women there are two possible outcomes of Chlamydia lower genital infection: spontaneous clearing of the infection without consequences and ascending of the infection to the upper genital tract and development of complication. Research showed that almost half of the women (44.7\%) with asymptomatic infection and therefore without antibiotic treatment will clear infection in one year [29]. In others who do not spontaneously clear the infection, bacteria can cause persistent infection and serious complications such as PID (Pelvic Inflammatory Disease), endometritis, salpingitis, ectopic pregnancy and tubal factor infertility (TFI), result of damaged fallopian tubes [30]. Costs associated with diagnosis and treatment of complications of Chlamydial urogenital infection pose a significant financial burden on healthcare, and on society as a whole, emphasizing its status of as a major public health issue.

\section{Immunogenetics of Chlamydia}

Human genetic variation is a vital causal factor of susceptibility to many common infectious diseases [31]. Immunogenetics is a field in biomedical research aiming at identifying immune related genes responsible for susceptibility to 
many autoimmune and infectious diseases [32]. It also assesses the role of genetic variations in genes responsible for host immune responses for the course and the outcome of infectious diseases.

Immunogenetic studies based on twin research for Chlamydia trachomatis estimated that $40 \%$ of variation in the characteristics of Chlamydia infection can be attributed to the differences in host genetic factors [33]. These differences are often determined by genetic variation, such as SNPs (Single Nucleotide Polymorphisms), in genes responsible for, amongst others, bacterial sensing receptors (and the pathways to which they belong) on cells such as macrophages as well as local vaginal and tubal epithelial cells. Bacterial sensing receptors, such as Toll Like Receptors (TLRs), the most studied of all the receptors of innate immune response [34], are responsible for adequate recognition of the pathogen and for the following adequate immune response. Genetic variations in genes encoding for these receptors can influence the susceptibility to Chlamydia, the course and the outcome of the infection in both positive and negative ways. Carrying certain SNPs could put an individual at high risk of persistent infection and at having increased risk for developing tubal pathology. A negative result for the same genetic markers means that a patient is not carrier, and thus is not highly susceptible to such a course of Chlamydia infection which results in tubal pathology [35]. Additionally, SNPs can also have protective role. Carrying certain SNPs can, indeed, protect its carriers from the development of tubal factor infertility. These different genetic markers need to be combined to provide an accurate assessment of risk factors and protective traits. Some of the key examples are described below.

\section{Chlamydia trachomatis host genetic factors}

Toll-like receptors (TLRs) have an important role in identifying pathogens and initiating innate immune response. They have been recognized as important factors in influencing differences in susceptibility to the course and outcome of Chlamydia infection [36, 37]. Immunogenetic research of Chlamydia focuses on TLR genes and genes involved in their pathways, assuming that SNPs in TLR genes can cause changes on the cell surface, either in the presentation of dysfunctional receptors or by changing the number and density of the receptors, thus leading to an inadequate immune response [38]. TLR2 showed an important role in the 
protection against late inflammatory sequelae following Chlamydia genital tract infection [37]. There is also a statistically significant association between certain TLR2 haplotypes and the protection from tubal pathology on one, and development of late inflammatory complications on the other side (the absence of TLR2 on the cell surface is associated with an increase in the severity of the Chlamydia infection) [39] Furthermore, there is an indication that certain SNPs in genes for TLR4 increase the risk for infertility as a late complication of Chlamydia infection. Nod Like Receptors (NODs) are intracellular pathogen recognition receptors (PRRs) with a role in recognition of Chlamydia, carrying polymorphism in genes responsible for NODs has an effect on the course and the outcome of Chlamydial infection [40].

Chemokine receptor CCR5, essential in the activation of T-cell immune response, has been indicated in the development of complications associated with Chlamydia infection. Research investigating the effect of $32 \mathrm{bp}$ deletion in CCR5 gene on the development of complications following $C$. trachomatis found that women with positive IgG for Chlamydia without TFI had higher incidence of CCR5delta 32 deletion compared to controls. Barr et al. demonstrated that this mutation has a protective effect against developing tubal pathology when both alleles are mutated [41]. CXCR5 chemokine receptor also appears to contribute to inter-individual differences in human tubal pathology following Chlamydial infection [42].

\section{Chlamydia induced infertility}

The most serious late complication of Chlamydial urogenital infection is infertility. It is estimated that $10-15 \%$ of couples worldwide have a problem conceiving, $30 \%$ of them have subfertility issues related to tubal patency [43]. Of all the cases of tubal factor infertility (TFI) $45 \%$ is related to Fallopian tubes damage by Chlamydia trachomatis infection [44]. Chlamydia infections are characterized by subtle and perfidious course and, if untreated, often leading to chronicity [45].

Infertility is defined as "any form of reduced fertility with prolonged time of unwanted non-conception" [46]. More precisely is defined as 1 year of unwanted non-conception with unprotected intercourse in the fertile phase of the menstrual cycles [43]. Most clinical guidelines for diagnosis and treatment of subfertility agree that the time required for the diagnosis of subfertility is 12 
months for couples of which the woman is less than 35 years old and 6 months in case the woman is older than 36.

The pathogenesis of TFI associated with Chlamydia trachomatis infection is not fully understood, however persistent infection is considered to be the most important mechanism behind it [38]. It is believed that multiple mechanisms of tissue damage are involved at different stages of pathology development. The immune response induced by persistent reaction either damages the tissue at the site of infection directly or indirectly by triggering autoimmune response [47].

Tubal damage and related infertility are conventionally diagnosed by hysterosalpingography (HSG), laparoscopy and Chlamydia Antibody Testing (CAT). The first two diagnostic procedures are costly and invasive. The third one is the most commonly used in the screening of tubal factor subfertility, its sensitivity for tubal pathology (of the most accurate CATs) is assessed at approximately $50-60 \%$, with a specificity of $85-90 \%$ [48]. These numbers indicate that there is space for misdiagnosis, where some CAT negative women have in fact tubal pathology, and a considerable part of the CAT positive patients do not.

Aforementioned immunogenetic research about the influence of genetic variations in genes coding for bacterial sensing receptors, if applied in assessing the risk of a persistent infection and development of infertility, could have potential to decrease the number of false negative and false positive women. Clinical relevance of the TFI screening test based on genomic markers would be in its potential of delaying or completely avoiding the use of expensive and invasive diagnostic procedures in patients with low risk of having tubal pathology and speeding up laparoscopy investigation in patients at high risk. 


\section{Aim of the thesis}

The aim of this thesis is to explore the translation of genomic information in the diagnosis and treatment of infectious diseases in general and to facilitate this translation in diagnosis of Chlamydia induced TFI in particular.

The first objective of the thesis is to provide an overview of the basic genomic and genetic findings with translational potential for application in diagnosis and treatment of STIs. The same section also aims at exploring the role of biobanks in basic research of infectious disease genomics and in this process of translation of this basic research into clinical practice. The second part of the thesis focuses on the introduction of genetic testing into diagnosis of chlamydia induced infertility for the purpose of improvement of predictive value of testing by combining genetic with other diagnostic procedures. Its objective is to assess the health need of infertile women in the Netherlands. Additionally it explores strategies for the introduction of genomic biomarkers into the diagnosis of infertility, which would meet those needs. Finally, it evaluates the attitudes of the Dutch gynecologist toward the implementation of the genomic biomarkers into routine subfertility diagnosis.

\section{Outline of the thesis}

Overall, Part 1 (Chapters 1 and 2) of the thesis describes the state of the art on Public Health Genomics of infectious diseases. Traditionally Public Health aims at understanding, treating, controlling and preventing infectious diseases[21] the relatively new field of Public Health Genomics expands on these activities and focuses on providing timely and credible information for effective and responsible translation of genomics research into health benefits of population. Chapter 1 provides insight into the translational potential of basic genomic and genetic findings for Human Immunodeficiency Virus (HIV), Human Papilloma Virus (HPV) and Chlamydia trachomatis genital tract infections, three sexually transmitted diseases of significant public health relevance. Genetic and genomic markers found to influence susceptibility and course of these infections are described, and their translational potential is outlined. Chapter $\mathbf{2}$ focuses on the role of biobanks in genomics research in Infectious diseases. It identifies 
the potential fields of interaction between infectious disease genomics and biobanks, with a goal of facilitating integration of genome-based knowledge into clinical practice.

Part 2 (Chapters 3, 4 and 5) of the thesis focuses on Chlamydia trachomatis induced infertility.

Chapter 3 provides an overview of the current state of the art on host genetic markers found to influence the susceptibility to, and the course of, Chlamydia trachomatis genitourinary infection and proposes a new clinical diagnostic approach in subfertility diagnostics based on this overview. It also describe how an integrated PHG model can be of value to see if these host genetic markers can be translated from the lab to the market and implemented into public health. Chapter 4 identifies clinical services currently in place in diagnosis and treatment of subfertility in the Netherlands, it defines the target population which could benefit from the addition of genetic testing in the diagnosis of infertility and assess their health needs. Finally, in this chapter the potential of genomic testing in meeting these health needs of the target population is explored.

Chapter 5 examines the attitudes of the Dutch gynaecologists towards the addition of genetic testing in the screening for TFI caused by Chlamydia trachomatis. In the first phase of the study we performed interviews with experienced reproductive specialists working in 4 Dutch Academic Hospitals. The results of the interviews were used to develop a questionnaire for the Dutch gynaecologists about their attitudes towards the addition of genetic testing in the diagnosis of TFI. In the second phase, using the questionnaire, we surveyed 48 doctors providing fertility care in 3 Dutch Academic hospitals in order to investigate what they perceive to be major challenges and major facilitating factors in introducing genetic testing in routine fertility work up. 


\section{REFERENCES}

1. Fontanarosa, PB, Pasche, B and DeAngelis, CD. Genetics and genomics for clinicians. The Journal of American Medical Association, 2008. 299(1 1): p. 1364-1365.

2. Khoury, MJ, Feero, WG, Reyes, M, et al. The Genomic Applications in Practice and Prevention Network. Genetic Medicine, 2009. 11(7): p. 488-494.

3. The Case for Personalized Medicine-4th Edition. 2014, Personalized Medicine Coalition. Available from: http://www. personalizedmedicinecoalition.org/Userfiles/PMCCorporate/file/pmc_ case_for_personalized_medicine.pdf.

4. Gwinn, M, Grossniklaus, DA, Yu, W, et al. Horizon scanning for new genomic tests. Genetic Medicine, 2011. 13(2): p. 161-165.

5. Deverka, PA, Schully, SD, Ishibe, N, et al. Stakeholder assessment of the evidence for cancer genomic tests: insights from three case studies. Genetic Medicine, 2012. 14(7): p. 656-662.

6. Khoury, MJ, Gwinn, M, Yoon, PW, et al. The continuum of translation research in genomic medicine: how can we accelerate the appropriate integration of human genome discoveries into health care and disease prevention? Genetics in medicine : official journal of the American College of Medical Genetics, 2007. 9(10): p. 665-674.

7. Khoury, MJ. Genome-Based Diagnostics: Clarifying Pathways to Clinical Use: Workshop Summary2012: The National Academies Press.

8. Schully, SD, Benedicto, CB, Gillanders, EM, et al. Translational research in cancer genetics: the road less traveled. Public Health Genomics, 2011. 14(1): p. 1-8.

9. BRCA1 and BRCA2: Cancer Risk and Genetic Testing. 2014, National Cancer Institute. Available from: http://www.cancer.gov/cancertopics/ factsheet/Risk/BRCA.

10. Schwarz, UI, Ritchie, MD, Bradford, Y, et al. Genetic Determinants of Response to Warfarin during Initial Anticoagulation. New England Journal of Medicine, 2008. 358(10): p. 999-1008.

11. Burke, W and Psaty, BM. PErsonalized medicine in the era of genomics. JAMA, 2007. 298(14): p. 1682-1684.

12. Enhancing the Oversight of Genetic Tests: Recommendations of the SACGT. 2000, National Institutes of Health. Available from: http:// osp.od.nih.gov/office-clinical-research-and-bioethics-policy/geneticshealth-and-society-secretarys-advisory-committee-genetic-testing/ enhancing-oversight-genetic-tests-recommendations-sacgt.

13. Badano, JL and Katsanis, N. Beyond Mendel: an evolving view of human genetic disease transmission. Nature Reviews Genetics, 2002. 3(10): p. 779-789. 
14. Manolio, TA, Brooks, LD and Collins, FS. A HapMap harvest of insights into the genetics of common disease. The Journal of Clinical Investigation, 2008. $118(5)$ : p. 1590-1605.

15. Mckeown, RE. The Epidemiologic Transition: Changing Patterns of Mortality and Population Dynamics. American Journal of Lifestyle Medicine, 2009. 3(1 Suppl): p. 19S-26S.

16. Patel, JN. Application of genotype-guided cancer therapy in solid tumors. Pharmacogenomics, 2013. 15(1): p. 79-93.

17. Manolio, TA, Chisholm, RL, Ozenberger, B, et al. Implementing genomic medicine in the clinic: the future is here. Genetic Medicine, 2013. 15(4): p. 258-267.

18. Worthey, EA, Mayer, AN, Syverson, GD, et al. Making a definitive diagnosis: Successful clinical application of whole exome sequencing in a child with intractable inflammatory bowel disease. Genetic Medicine, $2011.13(3)$ : p. 255-262.

19. Segal, S and Hill, AVS. Genetic susceptibility to infectious disease. Trends in Microbiology, 2003. 11 (9): p. 445-448.

20. Hill, AVS. Aspects of Genetic Susceptibility to Human Infectious Diseases. Annual Review of Genetics, 2006. 40(1): p. 469-486.

21. Fauci, AS and Morens, DM. The Perpetual Challenge of Infectious Diseases. New England Journal of Medicine, 2012. 366(5): p. 454-461.

22. Brand, A, Brand, $\mathrm{H}$ and Schulte in den Baumen, $\mathrm{T}$. The impact of genetics and genomics on public health. European Journal of Human Genetics, 2008. 16(1): p. 5-13.

23. Frodsham, AJ and Hill, AV. Genetics of infectious diseases. Human Molecular Genetics, 2004. 13 Spec No 2: p. R187-194.

24. Sexually transmitted infections in Europe 1990-2010. 2012, ECDC. Available from: http://www.ecdc.europa.eu/en/publications/ publications/201206-sexually-transmitted-infections-europe-2010. pdf.

25. Fenton, KA and Lowndes, CM. Recent trends in the epidemiology of sexually transmitted infections in the European Union. Sexually Transmitted Infections, 2004. 80(4): p. 255-263.

26. Prevalence and incidence of selected sexually transmitted infections Chlamydia trachomatis, Neisseria gonorrhoeae, Syphilis and Trichomonas vaginalis: 2005 estimates. 2011, WHO Report available from: http://www.who.int/reproductivehealth/publications/ rtis/9789241502450/en/.

27. Lanjouw, E, Ossewarde, JM, Stary, A, et al. 2010 European guideline for the management of Chlamydia trachomatis infections International Journal of STD \& AIDS, 2010. 21: p. 729-737.

28. Lyons, JM, Ouburg, S and Morré , SA. An integrated approach to Chlamydia trachomatis infection: the ICTI Consortium, an update. Drugs of Today, 2009. 45 Suppl B: p. 15-23. 
29. Morré , SA, van den Brule, AJ, Rozendaal, L, et al. The natural course of asymptomatic Chlamydia trachomatis infections: $45 \%$ clearance and no development of clinical PID after one-year follow-up. International Journal of STD \& AIDS, 2002. 13 Suppl 2: p. 12-18.

30. Paavonen, $\mathrm{J}$ and Eggert-Kruse, W. Chlamydia trachomatis: impact on human reproduction. Human Reproduction Update, 1999. 5(5): p. 433447.

31. Hill, AV. The immunogenetics of human infectious diseases. Annual Review of Immunology, 1998. 16: p. 593-617.

32. Hill, AVS. Immunogenetics and genomics. The Lancet, 2001.357(9273): p. 2037-2041.

33. Bailey, RL, Natividad-Sancho, A, Fowler, A, et al. Host genetic contribution to the cellular immune response to Chlamydia trachomatis: Heritability estimate from a Gambian twin study. Drugs of Today, 2009. 45 Suppl B: p. 45-50.

34. Fukata, M, Vamadevan, AS and Abreu, MT. Toll-like receptors (TLRs) and Nod-like receptors (NLRs) in inflammatory disorders. Seminars in Immunology, 2009. 21 (4): p. 242-253.

35. Morré , SA, Ouburg, S, Pena, AS, et al. The EU FP6 EpiGenChlamydia Consortium: contribution of molecular epidemiology and host-pathogen genomics to understanding Chlamydia trachomatis-related disease. Drugs of Today, 2009. 45 Suppl B: p. 7-13.

36. den Hartog, JE, Lyons, JM, Ouburg, S, et al. TLR4 in Chlamydia trachomatis infections: knockout mice, STD patients and women with tubal factor subfertility. Drugs of Today, 2009. 45 Suppl B: p. 75-82.

37. Darville, T, O’Neill, JM, Andrews, CW, Jr., et al. Toll-like receptor-2, but not Toll-like receptor-4, is essential for development of oviduct pathology in chlamydial genital tract infection. Journal of Immunology, 2003. 171(11): p. 6187-6197.

38. den Hartog, JE. Chlamydia trachomatis: Identifying women with tubal factor subfertility. Doctoral degree thesis. 2010. Maastricht University. Maastricht.

39. Karimi, O, Ouburg, S, de Vries, HJ, et al. TLR2 haplotypes in the susceptibility to and severity of Chlamydia trachomatis infections in Dutch women. Drugs of Today, 2009. 45 Suppl B: p. 67-74.

40. Morré, SA, Karimi, O and Ouburg, S. Chlamydia trachomatis: identification of susceptibility markers for ocular and sexually transmitted infection by immunogenetics. Fems Immunology and Medical Microbiology, 2009. 55(2): p. 140-153.

41. Barr, EL, Ouburg, S, Igietseme, JU, et al. Host inflammatory response and development of complications of Chlamydia trachomatis genital infection in CCR5-deficient mice and subfertile women with the CCR5delta32 gene deletion. Journal of Microbiology Immunology and Infection, 2005. 38(4): p. 244. 
42. Jiang, J, Karimi, O, Ouburg, S, et al. Interruption of CXCL13-CXCR5 axis increases upper genital tract pathology and activation of NKT cells following chlamydial genital infection. PLoS One, 2012. 7(11): p. e47487.

43. Evers, JL. Female subfertility. Lancet, 2002. 360(9327): p. 151-159.

44. Price, MJ, Ades, AE, Welton, NJ, et al. How much tubal factor infertility is caused by Chlamydia? Estimates based on serological evidence corrected for sensitivity and specificity. Sexually transmitted diseases, 2012. 39(8): p. 608-613.

45. Morrison, RP. New insights into a persistent problem - chlamydial infections. The Journal of Clinical Investigation, 2003. 111(11): p. 1647-1649.

46. Gnoth, C, Godehardt, E, Frank-Herrmann, P, et al. Definition and prevalence of subfertility and infertility. Human Reproduction, 2005. 20(5): p. 1144-1147.

47. LaVerda, D, Kalayoglu, MV and Byrne, Gl. Chlamydial heat shock proteins and disease pathology: new paradigms for old problems? Infectious Diseases in Obstetrics and Gynecology, 1999. 7(1-2): p. 64-71.

48. Land, JA, Van Bergen, JE, Morré , SA, et al. Epidemiology of Chlamydia trachomatis infection in women and the cost-effectiveness of screening. Human Reproduction Update, 2010. 16(2): p. 189-204. 

PART 1 



\section{CHAPTER 1}

Translational Potential into Health Care of Basic Genomic and Genetic Findings for Human Immunodeficiency Virus, Chlamydia trachomatis and human papillomavirus

J Malogajski, I Branković, SP Verweij, E Ambrosino, MA van Agtmael, A Brand, S Ouburg, S. A. Morré

BioMed Research International 2013: e892106 


\begin{abstract}
Individual variations in susceptibility to an infection as well as in the clinical course of the infection can be explained by pathogen related factors, environmental factors, and host genetic differences. In this paper we review the state-of-the-art basic host genomic and genetic findings' translational potential of human immunodeficiency virus (HIV), Chlamydia trachomatis (CT), and human papillomavirus (HPV) into applications in public health, especially in diagnosis, treatment, and prevention of complications of these infectious diseases. There is a significant amount of knowledge about genetic variants having a positive or negative influence on the course and outcome of HIV infection. In the field of Chlamydia trachomatis, genomic advances hold the promise of a more accurate subfertility prediction test based on single nucleotide polymorphisms (SNPs). In HPV research, recent developments in early diagnosis of infectioninduced cervical cancer are based on methylation tests. Indeed, triage based on methylation markers might be a step forward in a more effective stratification of women at risk for cervical cancer. Our review found an imbalance between the number of host genetic variants with a role in modulating the immune response and the number of practical genomic applications developed thanks to this knowledge.
\end{abstract}




\section{Introduction}

Infectious diseases represent a major health threat worldwide and a significant part of the burden of disease in developing countries [1]. Public health policy has traditionally had an important role in tackling such threat through established measures of prevention, mostly by controlling social and environmental determinants of health and through vaccination. With the recent advances in public health genomics, public health moved its focus from a "one size fits all" approach in health promotion and prevention activities to targeting populations and subpopulations with defined genetic risks and developed its unique role, translation of genome-based knowledge and technologies into public health policy and practice, and its integration across disciplines [2]. Scientific developments in basic research and the development of public health genomics have changed many paradigms regarding infectious diseases. Indeed, the recent evidence of genetic factors in the pathogenesis of infectious diseases transformed the view of such diseases from strictly pathogen-centric to the one incorporating host genetic determinants that modulate immune response. Though research in the field of genetic susceptibility to infectious diseases started in 1954, recent progress in genomics led to the characterization of molecular biomarkers and pathways as targets for diagnosis and intervention [3]. Furthermore, this understanding of infectious diseases explains the individual variation in susceptibility to an infection as well as the clinical course of the infection by pathogen related factors, environmental factors, and genetic differences. The field identifies genes responsible for influencing susceptibility to infections as well as their severity and response to treatment. This is predominantly achieved by studying candidate genes, genome wide associations, and twin studies [4]. A great amount of effort and resources have been directed to obtaining knowledge about host genetic components of infectious diseases and to confirm associations in order to develop genomic applications in everyday clinical practice and prevention. Nonetheless, although the amount of genetic data in relation to disease is increasing exponentially [5, 6], there is a clear lack of translation of such findings to healthcare applications. Indeed, the amount of information about basic genome-based scientific findings present in the scientific journals is disproportionate to the number of patents and marketed products used in hospitals [7]. In this paper, priority was given to 
three sexually transmitted diseases of significant public health relevance: HIV, HPV, and Chlamydia trachomatis (CT) genital tract infections. The aim of this review is to provide a state-of-the-art overview on the translational potential of basic genomic and genetic findings related to HIV, CT, and HPV infections, into applications in public health focusing on their diagnostics and treatment.

\section{Methods}

Based on our field of expertise in sexually transmitted diseases (STDs) we selected the most prevalent bacterial STD Chlamydia trachomatis (CT) and the 2 most prevalent viral STDs Human Papilloma Virus (HPV) and the Human Immunodeficiency Virus (HIV) knowing that from these infectious diseases human genetic and genomic markers are described.

We used the HuGE Navigator (Version 2.0: an integrated, searchable knowledge base of genetic associations and human genome epidemiology (http:// hugenavigator.net/)) [8] to identify papers with a description of potential translation on the basic findings of genetic and genomic markers into diagnostic applications and ultimately into public health. Identified papers and authors were expanded using PubMed searches. For each infectious disease a general introduction will be given, the key genetic and genomic markers will be described, and the translational potential outlined. Finally, a general discussion and conclusions will be provided.

\section{Results}

\section{HIV}

Despite the decrease in incidence of HIV infection (in 2009 the number of newly infected individuals dropped by almost $20 \%$ compared to the previous year), the prevalence of HIV is still very high. At the end of 2009 , it was estimated that there were 33.3 million people living with HIV. The growing prevalence and the reduction in the AIDS-related mortality are mainly attributed to the success of antiviral therapy [9]. Nonetheless, the public health relevance of the disease remains indisputable, as tackling HIV requires large financial expenditures, and it is still among the sexually transmitted diseases causing the highest morbidity and mortality and it is highly preventable [10]. As mentioned earlier, research in the 
field of infectious diseases has established that the susceptibility of an individual is also modulated by host genomic factors. In this context, recent genomic and genetic discoveries using candidate gene and genome wide association studies (GWAS) increased our knowledge of the association among genetic loci from the so-called "major susceptibility genes." HIV infection is the most studied infection by the aforementioned approaches. The research of a genetic role for the individual differences in the course of infection, besides offering new strategies for developing a treatment or a vaccine, also provides basic insights in the immunopathology of the infection. Moreover, this newly collected evidence could provide an opportunity of identifying persons at higher risk of getting or progression of the infection. On the other hand, this could detect patients having genes that make them long-term non-progressors, thus with delayed or no progression to AIDS.

\section{Review of the Host Genetic Variants Found to Influence HIV infection}

The review of papers written by the experts in the field of host genomic determinants of infection, disease progression, and disease outcome reveals the growing body of host genomic "suspects" by the year. However, few associations were positively confirmed. Among these, only $15-20 \%$ of observed genetic variants have been identified as influencing HIV infection [11]. Many studies and reviews place genetic variants of chemokine receptor and chemokine ligand genes, HLA and related genes on top of the list of influential genetic factors identified in HIV infection [11-16]. Chemokine receptors have an important role in modulating HIV-1 early infection. Particular attention has been given to CCR5 and CCR2 genes, encoding co- receptors on the surface of the CD4+ lymphocytes, crucial for HIV cell entry. In the initial stages of the infection, the HIV virus uses CCR5 as a preferred co -receptor [15]. As a result, a mutation in the chemokine receptor genes resulting in the absence or significant reduction of CCR5 molecules on the cell surface would have a protective effect. Indeed, the expression level of this co-receptor influences the HIV infection outcome, and mutation of this molecule is associated with the ability of the virus to enter the cells in vitro, the in vivo viral load, the CD4+ levels during highly active antiretroviral therapy (HAART: combination of three or more antiviral drugs), and the progression of the diseases to AIDS.

In 1996, it was discovered that the deletion of 32 base pairs of CCR5 $(C C R 5 \Delta 32)$ results in shortened and inactive proteins. So far, CCR5 $\Delta 32$ remains 
the only discovered mutation that completely protects homozygotes from HIV infection and in heterozygotes slows down the progression of the disease [1 1 ]. Moreover, the discovery of CCR5 $\triangle 32$ genetic variant opened the door for the development of a new type of anti-HIV medications. Data obtained fromCCR5 gene candidate studies have been rather timely applied in the pharmaceutical industry, leading to the development of novel therapies, as further discussed in the next section. In addition, the association between the $+190 \mathrm{~A}>\mathrm{G}$ mutation of CCR2 chemokine receptor and the delayed onset of AIDS was discovered in 1997. The resulting substitution of the amino acid Valine, at the position 64 of CCR2, to isoleucine influences HIV progression, but not the risk of HIV infection. HIV positive patients carrying this mutation showed delayed progression to AIDS by $2-4$ years [17].

\section{Application of Research Based on Chemokine Receptors}

As stressed earlier, the major goal of the research on host immunogenetics of $\mathrm{HIV}$ is to acquire knowledge of how differences in genetic variants are influencing individual susceptibility to infection and developing new drugs based on that. The research provided insights into the effects of CCR5 co-receptor blockade and down regulation on HIV infection [18].

As a result drugs with a new mechanism of action, the blockage of CCR5 receptors, were developed. These drugs are also known as entry inhibitors. So far there are only two approved such drugs in clinical use, Maraviroc (Pfizer) and Enfuvirtide (Roche) $[19,20]$. Of the two, Enfuvirtide was the first to be FDA approved. The success of this drug, despite its proven antiviral efficacy in patients' treatment, was constrained by the difficulties related to its subcutaneous administration, causing skin abscesses. The first orally administered HIV entry inhibitor was Maraviroc, approved by the FDA for patients with R5 virus types in 2006. The drug binds to the CCR5 chemokine receptor causing a conformational change that blocks the gp41-mediated fusion of viral and cellular membranes [19]. The next most promising HIV entry inhibitor is Vicriviroc (Schering-Plough), a medicine with the same action mechanism as Maraviroc, but expected to be more effective. Vicriviroc has still not been approved by FDA, but phase III clinical trials have been recently completed [21]. A recent extensive review of HIV-1 entry inhibitors patented from 2004-2010, [20], revealed 35 small CCR5 antagonist molecules patented by 5 different pharmaceutical companies (Astra Zeneca, ViroChem Pharma, Anormed, Inc./Genzyme Corp., Euroscreen, 
and Ono Pharmaceuticals). In the same review, it was found that the number of patents for CXCR4 (co-receptors for X4 HIV strains) antagonists and dual CCR5/CXCR4 antagonists is significantly lower. Further, clinical developments of CXR4 antagonists have been delayed in preclinical and clinical studies due to serious side effects (cardiac abnormalities and liver toxicity) or lack of drug efficacy. Human Leukocytes Antigen (HLA) genes encode proteins that present antigens to $T$ and $B$ lymphocytes. There are two classes of HLA genes: class I (loci A, B, and C) and class II genes. A strong association has been observed between HLAl alleles and protection/susceptibility to HIV [22]. The effect of HLA A, B, and C homozygosis in general is accelerated AIDS. Other confirmed associations include HLA alleles $B^{*} 27$ and $B * 57$ and delayed progression to AIDS $[15,16][22,23]$. On the other hand, the $B * 35$ allele is associated with increased susceptibility and more rapid progression of the disease. The median time in which homozygous carriers of the $B * 35$ allele develop AIDS is half the time of non-carriers of such alleles [24].

The association between genetic variants of HLA class I loci and CCR5 and the pathogenesis of HIV infection has been confirmed in recent years by many GWAS studies. However, GWAS did not identify further major susceptibility loci [25]. Association studies between HLA class II alleles and the susceptibility to the HIV infection has been less consistent. HLA genes have also been shown to have a role in the Mother to Child Transmission (MTCT) of HIV infection. Indeed, HLA class I concordance between mother and child is associated with higher risk of transmission, vice versa HLA discordance is associated with a lower risk [16].

\section{Application of Research on HLA Genes}

Although none of the mentioned HLA genes have yet been identified as a target for new drugs, the information gathered on the disease progression modulated by different genotypes has provided valuable information for clinical trials [22]. Research on HLA alleles led to important pharmacogenetic applications. HLA $B \star 5701$ positive patients, who are at risk for hypersensitivity to Abacavir (a nucleoside reverse transcriptase inhibitor), cannot be treated with this drug. This serious, and possibly fatal, adverse drug reaction is present in $5 \%$ of patients [26]. Genetic testing of all the individuals before prescribing the drug prevents serious side effects, building a very strong case for a stratified medicine approach, tailored to individual genetic characteristics. The idea behind it is that 
our personal genetic differences create a need for accordingly different treatment approaches. In the case of Abacavir recognizing interpersonal variation in reaction to drug is an excellent example of stratifying HIV treatment based on genetic research. In summary, HIV immunogenetic research provided some basic insights into the immunopathology of the infection and gave foundations to the development of new drugs for the therapy of the infection. Ideally this will be just the first step in advancing therapies. Information on individual susceptibility, higher or lower individual risks, and delayed or accelerated AIDS progression associated with certain gene variants will make a more individually tailored treatment possible in the future.

\section{Chlamydia trachomatis}

Chlamydia trachomatis is a leading cause for a variety of diseases including ocular, respiratory, and sexually transmitted diseases. This section of the review will only focus on the latter, since sexually transmitted Chlamydia infections are the most common worldwide, whereas, for instance, ocular infections are mostly seen in third world countries. Host genetic twin studies of Chlamydia have shown that $40 \%$ of the responses to Chlamydia are based on host genetics [27]. According to the WHO, "more cases of STD are caused by Chlamydia trachomatis than by any other bacterial pathogen" [28]. The persisting high incidence of 90-100 million cases per year worldwide makes Chlamydia trachomatis infection an enormous health problem throughout the world. The bacteria can be easily eliminated by antibiotic treatment; however, as a result of often being asymptomatic, the infection is frequently diagnosed too late or not at all. Infertility, premature delivery, PID, and ectopic pregnancy are some serious sequelae of the untreated infection [29]. Evaluation of the casual link between Chlamydia lower genital tract infection and tubal infertility is very challenging due to the fact that this is a "silent" complication, usually diagnosed years after the infection [30]. Infected women can either clear the bacteria without any damage to their reproductive functions or develop severe late complications, such as tubal occlusion and periadnexal adhesions, leading to infertility as the most severe of complications. The differences in disease outcome are often determined by genetic variations, such as single nucleotide polymorphisms (SNPs) in genes responsible for, amongst others, bacterial sensing receptors (and the pathways to which they belong) on cells such as macrophages as well as local vaginal and tubal epithelial cells. The higher the 
number of genes affected by SNPs, the more abnormal the immune response, leading to a higher chance of severe complications [31]. Inadequate recognition of the pathogen and consequent inadequate immune response lead to a higher risk of subfertility [32]. In a research performed on Gambian twins [27], it was estimated that $40 \%$ of variation in Chlamydia infection characteristics could be explained by differences in host genetic factors.

\section{Review of the Host Genetic Variants Found to Influence Chlamydia Lower Genital Tract Infection}

\section{TLR Receptors}

Toll-like receptors (TLRs), with their role in identifying pathogens and initiating innate immune response, have been recognized as the most important factors in influencing differences in susceptibility to course and outcome of Chlamydia infection [33, 34]. Indeed, much of immunogenetic research in this field is focused on TLR genes and genes involved in their pathways, not only by mRNAand protein-based studies but also by studying the association between SNPs in TLR genes leading to the loss of function of the receptors and the potential higher risk of late complications such as tubal infertility. The application of such research could be in the area of early diagnosis of tubal infertility or subfertility. Based on this evidence, the time now being lost as a result of late or misdiagnosis of tubal infertility could be directed to IVF attempts. So far, there are 10 TLRs identified in humans, recognizing different bacterial and viral components. TLRs activate signaling pathways of immune response against different pathogens by activating different inflammatory cytokines [35]. TLR2, TLR4, and TLR9 recognize pathogen-associated molecular patterns (PAMPs) of Chlamydia trachomatis. Genes for TLR receptors 2 and 4 are considered particularly important in modulating innate immune response to Chlamydia trachomatis [36]. Several studies showed that SNPs in TLR4 have a role in making women more prone to subfertility as a late complication of Chlamydia infection. Nonetheless, the exact role of TLR4 in subfertility has not been yet clearly understood [33, 34]. Subfertile women who have IgG antibodies for Chlamydia trachomatis have a two times higher likelihood to be carriers of the TLR4 +896 A allele, compared to women without tubal pathology [34]. Although this observation was not statistically significant, reported trends suggest that it could be worthwhile to further explore it in a larger cohort. Further, murine studies showed that TLR4 functional mice are more protected 
against reinfection compared with mice with dysfunctional or absent TLR4 [36]. In their study of genetic variants involved in the immune response regulation in genetic tract infections, Laisk et al. found that the TLR4 +896 A>G and +1 196 $\mathrm{C}>\mathrm{T}$ polymorphisms protect against multiple infections with $\mathrm{C}$. trachomatis, $\mathrm{N}$. gonorrhoeae, M. hominis, M. genitalium, U. parvum, and U. urealyticum.

Depending on the patient definition (i.e., including or excluding C. trachomatis serology), they found that specific MBL2 high producing haplotypes can have a protection of a risk effect in tubal factor infertility. Low-producing MBL2 haplotypes are associated with $C$. trachomatis serology positive tubal factor infertility patients [36]. In their study on the role of TLR2 and TLR4 in the development of tubal pathology on knock out (KO) mouse models, Darville et al. [33] showed that the amount of cytokines produced by macrophages depends on TLR2 but not on TLR4 receptors. Indeed, the deficiency of TLR2 receptors is associated with a decreased production of cytokines in vitro. In vivo, the deficiency or absence of TLR2 causes lower levels of inflammatory mediators, but the course of infection does not differ compared with naïve animals. Microscopic examination of the tubal tissue showed that mice with intact TLR2 are, however, more prone to the development of late inflammatory sequelae. Finally, their study concluded that TLR4 does not modulate innate immune response to Chlamydia, whereas in vivo experiments on TLR2 indicated its important role in protection against late inflammatory sequelae following Chlamydia genital tract infection [33]. In a study aiming at understanding the role of two TLR2 SNPs in the susceptibility to infection and contribution to the development of the tubal pathology in Dutch women, Karimi et al. [37] revealed a statistically significant association between certain TLR2 haplotypes and protection from tubal pathology and development of the late inflammatory complications (the absence of TLR2 is associated with an increase in the severity of the Chlamydia infection). As already mentioned, most of the studies assessing host genetic determinants of Chlamydia infections are focusing on the extracellular TLR2's and TLR4's contribution to the differences in the susceptibility and severity of the infection. However, there is also an interest in the relevance of the intracellular TLR9. So far, human cohort data have not shown significant differences between carriers of mutant alleles and controls in the susceptibility to infection, course of the infection, or frequency of later tubal pathology. On the other hand, experiments in mice models found that TLR9deficient mice had a higher level of protection against reinfection [38]. 


\section{HLA Alleles}

In addition to the research directed at TLR genes, there are also indications of association between tubal infertility caused by Chlamydia trachomatis and HLA alleles. Cohen et al. [39, 40] found that alleles of the HLA-DQ, DR 1, and DRB5 loci modulate the severity of Chlamydia infections. Kinnunen et al. also found that specific HLA-DQ alleles are more frequently present in women with tubal infertility [41].

Besides the TLR and HLA alleles, in 2009, Morré et al. published an extensive overview of the then known genetic variants influencing susceptibility and severity of Chlamydia infections including SNPs in cytokines and other pathogen recognition receptors like NODs [42].

\section{Application of Research.}

Immunogenetics research on Chlamydia trachomatis indicates that a proof of principle for the successful application of genetic and genomic markers for the prediction of late complications after the infection could have a strong public health impact. Subfertility poses an enormous burden on healthcare and society throughout the world. Worldwide, $15 \%$ of couples trying to conceive suffer from subfertility $[43,44]$. One of the major causes of female subfertility is tubal pathology (TP) [43], and CT is the single most common cause for infertility. If left untreated, CT may lead to ectopic pregnancy, tubal pathology, and ultimately infertility. The cost associated with subfertility is high, as it requires tubal surgery and in vitro fertilisation (IVF). Currently, CT IgG serology is used to assess the risk of CT-associated TP in subfertile women (20\%) (Figure 1) [45, 46].

CT serology has limited sensitivity and specificity and the predictive value is poor thus, many women undergo additional diagnostic procedures while not needed (40-45\%) or do not get intervention while needed (19\%). Laparoscopy is widely used to assess the risk of TP in women positive for CT IgG. 

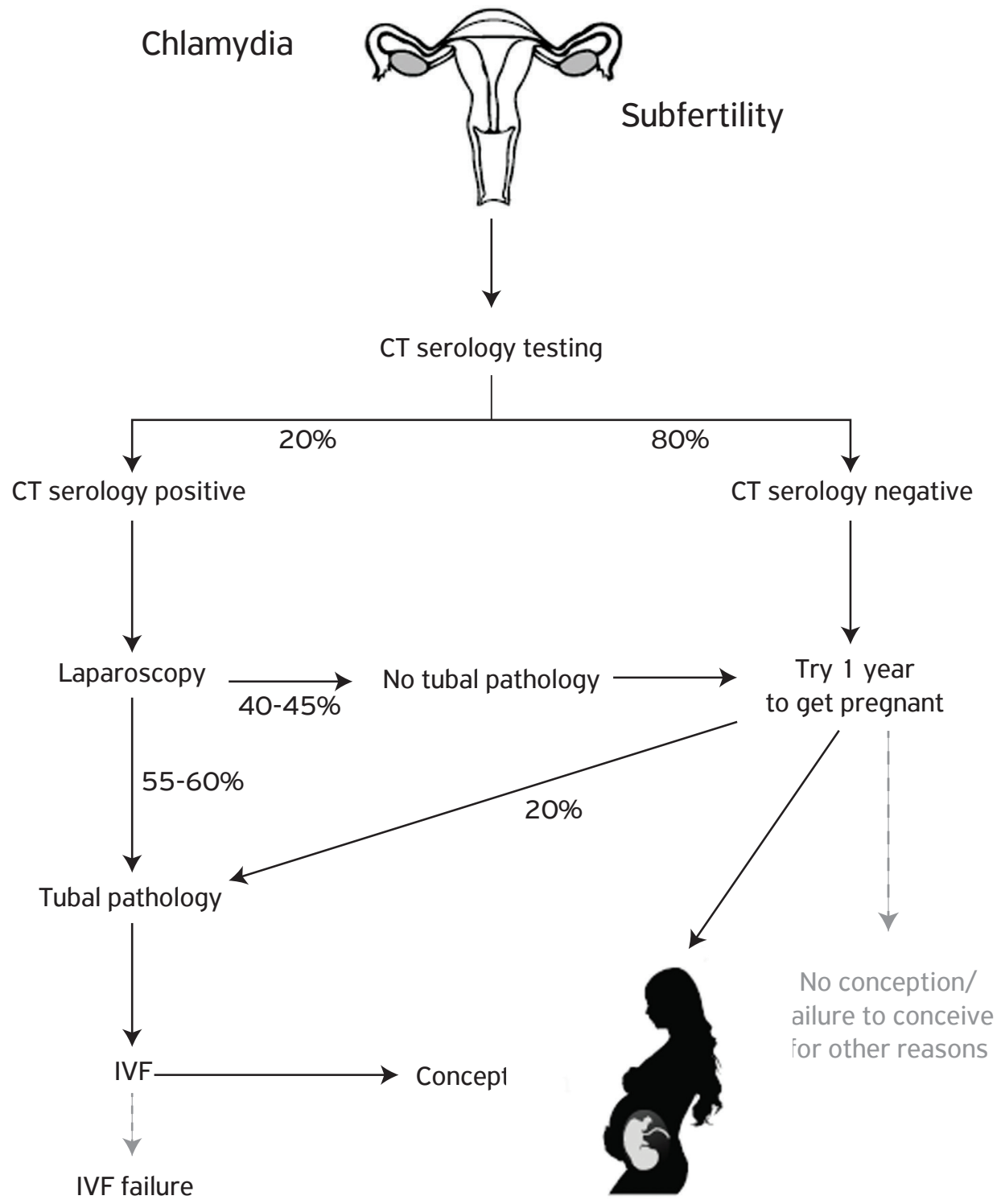

Figure 1: Current serology protocol for subfertility resulting from CT infection. Women with a negative CT serology are advised to try to conceive for one year; however, $20 \%$ of those women actually have tubal pathology and are thus misdiagnosed. Of the women with a positive CT serological test, 40$45 \%$ do not have tubal pathology after laparoscopic examination and are thus misdiagnosed. Figure adapted from Lal et al. [60]. 
This procedure is invasive and expensive (on average 3000 Euros including additional costs) and requires general anaesthesia. Furthermore, it holds a $1.5 \%$ risk of surgical complications (e.g., bleeding, infection, or worse). Therefore it is crucial to develop a companion diagnostic to improve the assessment of risk of TP in CT-positive and negative women. By doing so, one is able to prevent invasive procedures in patients without TP and reduce both the cost and the psychological burden associated with laparoscopy. This companion diagnostic should merge serology, taking into account serological positivity and titres and considering new serological responses (e.g., pgp3) [46] and add the predictive value of host genetic markers involved, for example, related to the innate immune response to pathogens. The genetic trait should consist of a series of markers with a so-called SNP load or gene load linked to decision making for performing laparoscopy or not. Future studies should be directed at performing studies in larger cohorts to access the true clinical potential of this approach.

\section{HPV}

Roughly $20 \%$ of cancers are linked to various infectious agents [47]. Human papilloma virus (HPV) is one of these agents, and the role of different HPV subtypes in the etiology of cervical cancer has been well established [48]. HPV infections are in most cases cleared by the actions of the immune system within one year and often remain asymptomatic throughout that period. However, a small percentage of the infections eventually lead to some form of cancer. HPV-induced cancers account for approximately one third of all cancers caused by infectious agents [49], and HPV is considered to be the most common sexually transmitted infectious agent [50]. However, studies have shown the existence of nonsexual modes of HPV transmission (including transplacental and transmission via fingers and objects [51-53]), and therefore, HPV cannot be referred strictly to as an STI [51].

The HPV virus infects skin or mucosal tissues in the anogenital area or the region of the head and neck. So far more than 100 types have been reported [49]. It has however been proven that approximately 15 out of these 100 types cause virtually all cases of cervical cancer [54]. Moreover, HPV types 16 and 18 account for around $70 \%$ of cervical cancer cases, and they-particularly type 16-have also been identified in anal, as well as some head and neck cancers [55]. The strong association between HPV infection and cervical carcinogenesis makes cervical cancer preventable, thus fulfilling an important 
criterion for public health relevancy. With the introduction of HPV vaccines, a major breakthrough in prevention has been made. Vaccines proved to be safe and efficacious [56] and vaccination programmes for girls and young women have been implemented in many countries.

\section{Review of the Host Genetic Variants Found to Influence HPV Infection.}

Of all the women who are infected with HPV, only a small percentage develops cervical cancer. This observation suggests a role of host genetic factors influencing persistent HPV infections and progression into cervical cancer.

The Role of HLA. Alleles have been reported to be associated with the development of HPV-related cervical cancer. In their review of evaluating this association, Hildesheim and Wang $[57,58]$ found several alleles of HLA class II to be associated with higher risk of developing cervical cancer (DQB $1{ }^{*} \mathrm{O} 3$ alleles and DRB 1 * 1501 , DQB $1{ }^{*}$ 0602). As for HLA genetic variants' protective effect, several studies consistently reported that DRB 1 * 13 and DOB 1 *0603 are associated with it $[57,58]$. Associations between HLA and HPV infection and progression to cancer are reported to be population- and HPV typedependent. Indeed, HLA DQB 1 *030 1 allele carries an increased risk of cervical cancer in the British population in case of infection with all HPV subtypes [58], while researchers in Bolivia found a statistically significant association of HLA DRB 1 * 1602 with susceptibility to infection [59].

In their recently published review of the genetic susceptibility to cervical cancer, Chen et al. [61] presented the most important genetic polymorphisms associated with the development of this disease. Their literature search identified, in addition to HLA genetic variants, genes encoding interleukin- $1 \beta$, tumor necrosis factor $\alpha$, interleukin-2 A and B, interferon- $\gamma$, interleukin-10, cytotoxic t-Lymphocyte antigen-4, p53, BRCA 1, and LAMB3 as genes associated with persistent HPV infection and progression to cervical cancer [61]. In addition, certain genes encoding killer immunoglobulin-like receptors (KIR) also seem to be associated with cervical cancer [62].

So far, no genetic or genomic applications have been developed based on these findings. When it comes to applying genetic knowledge and discoveries into the field of HPV infection and cervical findings diagnosis and prevention, the strategy known as methylation takes the lead. 


\section{The Role of Methylation}

Methylation is a common mechanism through which the silencing of genes, and among these tumor-suppressor genes, can be achieved [63]. It represents a chemical alteration in regions of DNA referred to as "CpG islands," commonly found in many promoter regions. The alteration leads to the inhibition of the transcription of genes controlled by such methylated promoters [64]. Methylation markers are easily detected in cervical scrapes, with, for example, methylation-specific PCR (MSP). Hence, positive MSP results in these samples are indicators of methylation of relevant genes in the tissue [64]. At the moment, the strategy for early detection of cervical neoplasia in screening programmes is cervical scraping cytomorphologic assessment (PAP test), which has a considerably low sensitivity. Data on sensitivity and specificity of the PAP test are highly heterogeneous. Depending on the study done and combination of tests and reference standard thresholds applied, they range from 18\% to $98 \%$ for sensitivity and from $17 \%$ to $99 \%$ for specificity [65]. Furthermore, the National Cancer Institute assessed the sensitivity of the PAP smear to be 55$80 \%$ for high grade lesions and around $68 \%$ for low grade lesions [66]. Taking this into consideration, there is a need for the development of novel approaches, and additional tools based on methylation markers might be a step forward.

\section{Application of Methylation in Triage of Cervical Carcinomas.}

In the study by Henken et al. [64], 29 tumor-suppressor genes were analyzed as potential methylation targets, and 12 of them were found to have methylated gene promoters in cervical cancer tissue. Eight of those were also associated with consecutive stages in HPV-mediated transformation in vitro. The promoter that was most commonly methylated (in $92 \%$ of the examined carcinoma samples) was MGMT.

Methylation of the promoters CCNA1 and C130RF18 in cervical scrapings is found to be strongly associated $(P<0.0005)$ with CIN2 (moderate cervical intraepithelial dysplasia) and higher grade stages of cervical dysplasia, as was determined in the study by Yang et al [67]. Hence, these would be suitable markers for a triage test, referring a patient to a gynecologist upon a methylationpositive result. The more severe the lesion in the sample, the more methylation was present in these two gene promoters. 


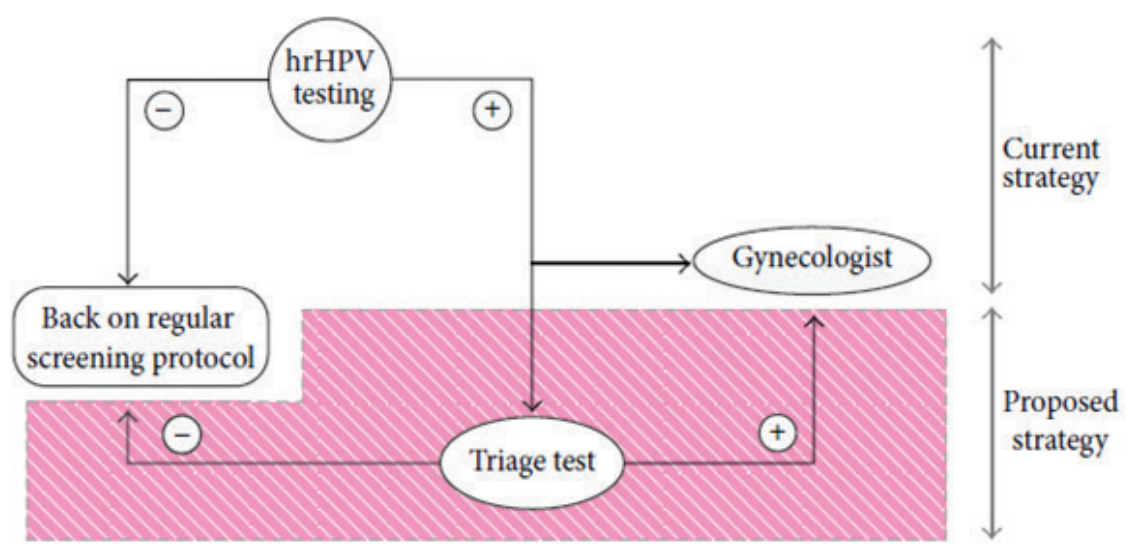

Proposal part for the introduction of the triage test

Figure 2: Introducing methylation as an addition to the primary hrHPV test would lower the number of unnecessary referrals to gynaecologists. Figure based on Yang et al. [67].

Analysis of high methylation of these two markers has a high specificity (96\% and $100 \%$, resp.), as well as high positive predictive value.

Further, Yang et al. [67] suggest that their methylation test should be used as a triage test in primary hrHPV testing (high risk HPV test identifies types of HPV which are linked to cervical cancer). hrHPV testing is more effective in preventing invasive cervical cancer; however, it is considered to be less sensitive than cytology in detecting CINs. Introducing methylation as a part of a triage test to the primary hrHPV test would lower the number of unnecessary referrals to gynaecologists; especially in younger women who tend to be over diagnosed [68] (see Figure 2).

In another study evaluating the potential value of the methylation markers CADM 1 and MAL as a triage tool for hrHPV+ women, it was found that there is a solid reasoning for combining markers which relate to different stages in cervical carcinogenesis [69]. They examined and confirmed the advantage of combining methylation patterns in the promoter region of more than one suppressor gene with the aim to increase the sensitivity for high grade CINs. A methylation-based test focuses on later phases of the carcinogenesis, given that these promoter alterations increase in these late stages. However, methylationdriven silencing of MAL promoter takes place at a very early point, before HPV- 
positive keratinocytes undergo tumor transformation. Whereas, silencing of CADM 1 promoter by methylation correlates more with late stages. Overmeer et al. demonstrated that this marker combination is optimal for detection of CIN3 lesions [69]. In the process of progression into late stages, there are genes other than oncogenes and tumor suppressors also relevant. MicroRNAs (miRNAs) are short noncoding RNA molecules, which act in regulating expression of protein coding genes, by pairing with sequences within such genes. hsa-miR-124 is an miRNA known to be silenced by methylation in many cancers, and Wilting et al. (2010) proved that this mode of silencing frequently occurs in cervical lesions as well [70]. No methylation was found in normal tissues, while almost $60 \%$ was detected in CIN3 lesions, and more than 93\%methylation of hsa-miR-124 was present in cervical carcinomas. The methylation of this gene is not directly related to the presence of hrHPV. High positivity is however observed in CIN3 and cervical carcinomas, which altogether makes it a potentially very useful triage marker for hrHPV positive women. This applies however not for setting where HPV genotyping is not implemented yet including under development countries. Triage could serve as an additional step that would more aptly bridge screening and diagnosis in order for a better stratification of women at risk to be achieved [7 1]. It would be used on those with positive primary screening results to determine the further risk of the progression into later stages. The effects of constructing this type of triage test based on methylation would be expected to land a formidable impact on policies that currently regulate screening intervals. 


\section{Discussion and Conclusion}

To our knowledge, this is the first review on the translational potential of basic genomic and genetic findings for HIV, CT, and HPV into applications in public health and in diagnostics, treatment, and prevention of late complications of these infectious diseases. We found scarce examples of the current application of genomic/genetic findings, in pharmacogenomics, and we found examples of genomic information with a promise of translation in the near future. In our review, we did not focus on analytic validity, clinical validity, and clinical utility and other criteria generally considered to be the most important factors in evaluation of the genetic/genomic applications [72]. Since there are still no market-ready applications, so the aforementioned criteria could not be considered; we focused on an earlier step of this process. We focused on the promising examples of translation of the discovery into a possible application. Based on the review of the relevant literature some examples can be considered promising. The genes responsible for susceptibility to HIV infection can be basically divided in two groups, chemokine receptors genes and HLA genes. So far, the discovery of the CCR5 $\Delta 32$ genetic variant opened the door for the development of new anti-HIV drugs. Although undoubtedly a very important step forward, CCR5 targeted therapy and the research behind it are just one of the possible applications of immunogenetic information. Indeed, there is a significant amount of knowledge of certain genetic variants having a positive or negative influence on the course and outcome of HIV infection. Possible future use of the knowledge about the expected course of the infection would be advancing the standard of care and therapy after routine genetic testing.

In the field of Chlamydia trachomatis caused subfertility there is a promise for a more accurate subfertility diagnosis based on SNPs. Research showed that SNPs in TLR4 possibly increase the risk of tubal pathology. Specific TLR2 haplotypes are associated with protection from tubal pathology and development of the late inflammatory complications. These findings, together with the one carrying multiple SNPs in multiple pattern recognition receptors' (PRRs) encoding genes (TLR9, TLR4, CD 14, and CARD 15/NOD2) doubles the risk of tubal pathology in Chlamydia trachomatis IgG-positive women compared to IgG-positive women carrying less than two SNPs, offer a proof of concept for the development of a genomic application in diagnosis of subfertility. A genetic test as a part 
of routine subfertility diagnosis should be able to save time and money by decreasing the number of unnecessary laparoscopies and the time patients unsuccessfully spend trying to get pregnant. In the field of HPV, there are some promising advancements in the early diagnosis of cervical cancer based on methylation tests. The methylation markers CADM 1 and MAL were found to be an optimal combination for the detection of CIN3 lesions [69]. Moreover, the methylation of CCNA1 and C130RF18 in cervical scrapings is found to be strongly associated with CIN2 and higher grade stages [67]. A triage test based on such methylation markers might be an important step towards a more effective stratification of patients at risk for cervical cancer. The knowledge about the gene-disease associations should lead to growing numbers of genetic tests, which will in the future have an increasingly important role, in tailored clinical and drug treatment. However, in order for this translation process to succeed, the wide consensus among scientists, clinicians, policy makers, and the industry on necessity of going in this direction needs to be achieved [73]. Based on what we have shown here, there are many host genetic variants found to have a role in modulating the immune response to HIV, HPV, and Chlamydia infections. However, we found an imbalance between the number of host genetic variants with a role in modulating the immune response and the number of practical genomic applications. Thus, such new knowledge and technologies from basic research are not yet integrated in health in a timely, effective, and efficient manner [7].

This imbalance, the lack of translation from bench to bedside, is in favor of basic research that seems to be somewhat hermetic in quality, revealing confirmed positive association with a certain genetic variant and not exploring the future implications of these findings, should not represent a norm in the field. The next step is needed in which gene-disease association leads to the development of the genetic/genomic application. Starting with interdisciplinary collaboration is very important in the process of evaluation of role of genetic variants in the etiology of human diseases [74]. There are some clear and well-supported genetic associations with particular infectious diseases; these should be driving forces of the successful translation process. 


\section{REFERENCES}

1. Frodsham, AJ and Hill, AV. Genetics of infectious diseases. Human Molecular Genetics, 2004. 13 Spec No 2: p. R187-194.

2. Burke, W, Khoury, MJ, Stewart, A, et al. The path from genome-based research to population health: development of an international public health genomics network. Genetics in Medicine, 2006. 8(7): p. 451458.

3. Hill, AV. Aspects of genetic susceptibility to human infectious diseases. Annual Review of Genetics, 2006. 40: p. 469-486.

4. Hill, AV. Immunogenetics and genomics. The Lancet, 2001. 357(9273): p. 2037-2041.

5. Rowell, JL, Dowling, NF, Yu, W, et al. Trends in population-based studies of human genetics in infectious diseases. PLoS One, 2012. 7(2): p. e25431.

6. Khoury, MJ, Gwinn, M, Clyne, M, et al. Genetic epidemiology with a capital $\mathrm{E}$, ten years after. Genetic Epidemiology, 2011 . 35(8): p. 845-852.

7. Lal, JA, Schulte In den Baumen, T, Morré , SA, et al. Public health and valorization of genome-based technologies: a new model. Journal of Translational Medicine, 201 1. 9: p. 207.

8. Yu, W, Gwinn, M, Clyne, M, et al. A navigator for human genome epidemiology. Nature Genetics, 2008. 40(2): p. 124-125.

9. UNAIDS Report on the global AIDS epidemic 2010. UNAIDS. Available from: http://www.unaids.org/globalreport/global report.htm.

10. Updated Guidelines for Evaluating Public Health Surveillance Systems 2001, Center for Disease Control. Recommendations from the Guidelines Working Group, Available from: http://www.cdc.gov/mmwr/ preview/mmwrhtml/rr5013a1.htm. 11. An, P and Winkler, CA. Host genes associated with HIV/AIDS: advances in gene discovery. Trends in Genetics, 2010. 26(3): p. 119-131.

12. Tang, $\mathrm{J}$ and Kaslow, RA. The impact of host genetics on HIV infection and disease progression in the era of highly active antiretroviral therapy. AIDS, 2003. 17 Suppl 4: p. S51-60.

13. He, W, Castiblanco, J, Walter, EA, et al. Mendelian randomization: potential use of genetics to enable causal inferences regarding HIVassociated biomarkers and outcomes. Current Opinon in HIV and AIDS, 2010. 5(6): p. 545-559.

14. O'Brien, SJ, Nelson, GW, Winkler, CA, et al. Polygenic and multifactorial disease gene association in man: Lessons from AIDS. Annual Review of Genetics, 2000. 34: p. 563-591.

15. Kaslow, RA, Dorak, T and Tang, JJ. Influence of host genetic variation on susceptibility to HIV type 1 infection. Journal of Infectious Diseases, 2005. 191 Suppl 1: p. S68-77. 
16. Singh, KK and Spector, SA. Host genetic determinants of human immunodeficiency virus infection and disease progression in children. Pediatric Research, 2009. 65(5 Pt 2): p. 55R-63R.

17. Smith, MW, Dean, M, Carrington, $M$, et al. Contrasting genetic influence of CCR2 and CCR5 variants on HIV- 1 infection and disease progression. Hemophilia Growth and Development Study (HGDS), Multicenter AIDS Cohort Study (MACS), Multicenter Hemophilia Cohort Study (MHCS), San Francisco City Cohort (SFCC), ALIVE Study. Science, 1997. 277(5328): p. 959-965.

18. Hutter, $\mathrm{G}$ and Ganepola, S. The CCR5-delta32 polymorphism as a model to study host adaptation against infectious diseases and to develop new treatment strategies. Experimental Biology and Medicine (Maywood), 2011. 236(8): p. 938-943.

19. McKinnell, JA and Saag, MS. Novel drug classes: entry inhibitors [enfuvirtide, chemokine (C-C motif) receptor 5 antagonists]. Current Opinion in HIV and AIDS, 2009. 4(6): p. 513-517.

20. Singh, IP and Chauthe, SK. Small molecule HIV entry inhibitors: Part I. Chemokine receptor antagonists: 2004 - 2010. Expert Opinon on Therapeutic Patents, 2011 . 21(2): p. 227-269.

21. Gilliam, BL, Riedel, DJ and Redfield, RR. Clinical use of CCR5 inhibitors in HIV and beyond. Journal of Translational Medicine, 2011. 9 Suppl 1: p. S9.

22. Carrington, $M$ and O'Brien, SJ. The influence of HLA genotype on AIDS. Annual Review of Medicine, 2003. 54: p. 535-551.

23. den Uyl, D, van der Horst-Bruinsma, IE and van Agtmael, M. Progression of HIV to AIDS: a protective role for HLA-B27? AIDS Reviews, 2004. 6(2): p. 89-96.

24. O'Brien, SJ and Nelson, GW. Human genes that limit AIDS. Nature Genetics, 2004. 36(6): p. 565-574.

25. Chapman, SJ and Hill, AV. Human genetic susceptibility to infectious disease. Nature Reviews Genetics, 2012. 13(3): p. 175-188.

26. Mallal, S, Nolan, D, Witt, C, et al. Association between presence of HLA-B*5701, HLA-DR7, and HLA-DQ3 and hypersensitivity to HIV-1 reverse-transcriptase inhibitor abacavir. The Lancet, 2002. 359(9308): p. 727-732.

27. Bailey, RL, Natividad-Sancho, A, Fowler, A, et al. Host genetic contribution to the cellular immune response to Chlamydia trachomatis: Heritability estimate from a Gambian twin study. Drugs Today (Barc), 2009. 45 Suppl B: p. 45-50.

28. Sexually Transmitted Diseases. Disease burden. WHO.

29. Starnbach, MN and Roan, NR. Conquering sexually transmitted diseases. Nature Reviews Immunology, 2008. 8(4): p. 313-317. 
30. Land, JA, Van Bergen, JE, Morré, SA, et al. Epidemiology of Chlamydia trachomatis infection in women and the cost-effectiveness of screening. Human Reproduction Update, 2010. 16(2): p. 189-204.

31. den Hartog, JE, Ouburg, S, Land, JA, et al. Do host genetic traits in the bacterial sensing system play a role in the development of Chlamydia trachomatis-associated tubal pathology in subfertile women? BMC Infectious Diseases, 2006. 6: p. 122.

32. den Hartog, JE, Morré , SA and Land, JA. Chlamydia trachomatisassociated tubal factor subfertility: Immunogenetic aspects and serological screening. Human Reproduction Update, 2006. 12(6): p. 719-730.

33. Darville, T, O’Neill, JM, Andrews, CW, Jr., et al. Toll-like receptor-2, but not Toll-like receptor-4, is essential for development of oviduct pathology in chlamydial genital tract infection. Journal of Immunology, 2003. 171(11): p. 6187-6197.

34. den Hartog, JE, Lyons, JM, Ouburg, S, et al. TLR4 in Chlamydia trachomatis infections: knockout mice, STD patients and women with tubal factor subfertility. Drugs Today (Barc), 2009. 45 Suppl B: p. 7582.

35. Kawai, T and Akira, S. TLR signaling. Cell Death \& Differentiation, 2006. 13(5): p. 816-825.

36. Laisk, T, Peters, M, Saare, M, et al. Association of CCR5, TLR2, TLR4 and MBL genetic variations with genital tract infections and tubal factor infertility. Journal of Reproductive Immunology, 2010.87(1-2): p. 7481.

37. Karimi, O, Ouburg, S, de Vries, HJ, et al. TLR2 haplotypes in the susceptibility to and severity of Chlamydia trachomatis infections in Dutch women. Drugs Today (Barc), 2009. 45 Suppl B: p. 67-74.

38. Ouburg, S, Lyons, JM, Land, JA, et al. TLR9 KO mice, haplotypes and CPG indices in Chlamydia trachomatis infection. Drugs Today (Barc), 2009. 45 Suppl B: p. 83-93.

39. Cohen, CR, Sinei, SS, Bukusi, EA, et al. Human leukocyte antigen class II DQ alleles associated with Chlamydia trachomatis tubal infertility. Obstetrics \& Gynecology, 2000. 95(1): p. 72-77.

40. Cohen, CR, Gichui, J, Rukaria, R, et al. Immunogenetic correlates for Chlamydia trachomatis-associated tubal infertility. Obstetrics and gynecology, 2003. 101(3): p. 438-444.

41. Kinnunen, AH, Surcel, HM, Lehtinen, M, et al. HLA DQ alleles and interleukin-10 polymorphism associated with Chlamydia trachomatisrelated tubal factor infertility: a case-control study. Human Reproduction, 2002. 17(8): p. 2073-2078. 
42. Morré, SA, Karimi, $\mathrm{O}$ and Ouburg, S. Chlamydia trachomatis: identification of susceptibility markers for ocular and sexually transmitted infection by immunogenetics. FEMS immunology and medical microbiology, 2009. 55(2): p. 140-153.

43. Evers, JL. Female subfertility. The Lancet, 2002. 360(9327): p. 151159.

44. Broeze, KA, Opmeer, BC, van der Veen, F, et al. Individual patient data meta-analysis: a promising approach for evidence synthesis in reproductive medicine. Human Reproduction Update, 2010. 16(6): p. 561-567.

45. Broeze, KA, Opmeer, BC, Coppus, SF, et al. Chlamydia antibody testing and diagnosing tubal pathology in subfertile women: an individual patient data meta-analysis. Human Reproduction Update, 2011 . 17(3): p. 301-310.

46. Wills, GS, Horner, PJ, Reynolds, R, et al. Pgp3 antibody enzymelinked immunosorbent assay, a sensitive and specific assay for seroepidemiological analysis of Chlamydia trachomatis infection. Clinical and Vaccine Immunology, 2009. 16(6): p. 835-843.

47. Roman, E, Simpson, J, Ansell, P, et al. Childhood acute lymphoblastic leukemia and infections in the first year of life: a report from the United Kingdom Childhood Cancer Study. American Journal of Epidemiology, 2007. 165(5): p. 496-504.

48. An, HJ, Kim, KR, Kim, IS, et al. Prevalence of human papillomavirus DNA in various histological subtypes of cervical adenocarcinoma: a population-based study. Modern Pathology, 2005. 18(4): p. 528-534.

49. Lehoux, M, D'Abramo, CM and Archambault, J. Molecular mechanisms of human papillomavirus-induced carcinogenesis. Public Health Genomics, 2009. 12(5-6): p. 268-280.

50. Baseman, JG and Koutsky, LA. The epidemiology of human papillomavirus infections. Journal of Clinical Virology, 2005. 32 Suppl 1: p. S16-24.

51. Pao, CC, Tsai, PL, Chang, YL, et al. Non-sexual papillomavirus transmission routes. The Lancet, 1992. 339(8807): p. 1479-1480.

52. Burchell, AN, Winer, RL, de Sanjose, S, et al. Chapter 6: Epidemiology and transmission dynamics of genital HPV infection. Vaccine, 2006. 24 Suppl 3: p. S3/52-61.

53. Tay, SK. Genital oncogenic human papillomavirus infection: a short review on the mode of transmission. Annals of the Academy of Medicine, Singapore, 1995. 24(4): p. 598-601.

54. Schiffman, $M$ and Castle, PE. Human papillomavirus: epidemiology and public health. Archives of Pathology \& Laboratory Medicine, 2003. 127(8): p. 930-934.

55. Syrjanen, K. Mechanisms and predictors of high-risk human papillomavirus (HPV) clearance in the uterine cervix. European Journal of Gynaecological Oncology, 2007. 28(5): p. 337-351. 
56. Harper, DM. Prevention of human papillomavirus infections and associated diseases by vaccination: a new hope for global public health. Public Health Genomics, 2009. 12(5-6): p. 319-330.

57. Hildesheim, A and Wang, SS. Host and viral genetics and risk of cervical cancer: a review. Virus Research, 2002. 89(2): p. 229-240.

58. Cuzick, J, Terry, G, Ho, L, et al. Association between high-risk HPV types, HLA DRB 1 * and DQB 1 * alleles and cervical cancer in British women. British Journal of Cancer, 2000. 82(7): p. 1348-1352.

59. Cervantes, J, Lema, C, Valentina Hurtado, L, et al. HLA-DRB $1{ }^{*} 1602$ allele is positively associated with HPV cervical infection in Bolivian Andean women. Human Immunology, 2003. 64(9): p. 890-895.

60. Lal, JA, Malogajski, J, Verweij, SP, et al. Chlamydia trachomatis infections and subfertility: opportunities to translate host pathogen genomic data into public health. Public Health Genomics, 2013. 16(1-2): p. 50-61.

61. Chen, X, Jiang, J, Shen, $\mathrm{H}$, et al. Genetic susceptibility of cervical cancer. Journal of Biomedical Research, 201 1. 25(3): p. 155-164.

62. Arnheim, L, Dillner, J and Sanjeevi, CB. A population-based cohort study of KIR genes and genotypes in relation to cervical intraepithelial neoplasia. Tissue Antigens, 2005. 65(3): p. 252-259.

63. Esteller, $M$ and Herman, JG. Cancer as an epigenetic disease: DNA methylation and chromatin alterations in human tumours. The Journal of Pathology, 2002. 196(1): p. 1-7.

64. Henken, FE, Wilting, SM, Overmeer, RM, et al. Sequential gene promoter methylation during HPV-induced cervical carcinogenesis. British Journal of Cancer, 2007. 97(10): p. 1457-1464.

65. Nanda, K, McCrory, DC, Myers, ER, et al. Accuracy of the Papanicolaou test in screening for and follow-up of cervical cytologic abnormalities: a systematic review. Annals of Internal Medicine, 2000. 132(10): p. $810-$ 819.

66. Cervical Cancer Screening. NIH. Available from: http://www.cancer.gov/ cancertopics/pdq/screening/cervical/HealthProfessional.

67. Yang, N, Eijsink, JJ, Lendvai, A, et al. Methylation markers for CCNA 1 and C130RF18 are strongly associated with high-grade cervical intraepithelial neoplasia and cervical cancer in cervical scrapings. Cancer Epidemiology, Biomarkers Prevention, 2009. 18(11): p. 3000-3007.

68. Ronco, G, Giorgi-Rossi, P, Carozzi, F, et al. Efficacy of human papillomavirus testing for the detection of invasive cervical cancers and cervical intraepithelial neoplasia: a randomised controlled trial. The Lancet Oncology, 2010. 11 (3): p. 249-257.

69. Overmeer, RM, Louwers, JA, Meijer, CJ, et al. Combined CADM1 and MAL promoter methylation analysis to detect (pre-)malignant cervical lesions in high-risk HPV-positive women. International Journal of Cancer, 2010. 
70. Wilting, SM, van Boerdonk, RA, Henken, FE, et al. Methylation-mediated silencing and tumour suppressive function of hsa-miR-124 in cervical cancer. Molecular Cancer, 2010. 9: p. 167.

71. Solomon, D. Chapter 14: Role of triage testing in cervical cancer screening. Journal of the National Cancer Institute Monographs, 2003(31): p. 97-101.

72. Teutsch, SM, Bradley, LA, Palomaki, GE, et al. The Evaluation of Genomic Applications in Practice and Prevention (EGAPP) Initiative: methods of the EGAPP Working Group. Genetics in Medicine, 2009. 11(1): p. 3-14.

73. Burke, W, Atkins, D, Gwinn, M, et al. Genetic test evaluation: information needs of clinicians, policy makers, and the public. American Journal of Epidemiology, 2002. 156(4): p. 311-318.

74. Little, J, Bradley, L, Bray, MS, et al. Reporting, appraising, and integrating data on genotype prevalence and gene-disease associations. American Journal of Epidemiology, 2002. 156(4): p. 300-310. 



\section{CHAPTER 2}

Biobanking and translation of human genetics and genomics for infectious diseases

I Branković*, J Malogajski*, SA. Morré

Applied \& Translational Genomics 2014, 3(2): 30-35

* These authors contributed equally to the manuscript 


\begin{abstract}
Biobanks are invaluable resources in genomic research of both the infectious diseases and their hosts. This article examines the role of biobanks in basic research of infectious disease genomics, as well as the relevance and applicability of biobanks in the translation of impending knowledge and the clinical uptake of knowledge of infectious diseases. Our research identifies potential fields of interaction between infectious disease genomics and biobanks, in line with global trends in the integration of genome-based knowledge into clinical practice. Furthermore, it examines various networks and biobanks that specialize in infectious diseases (including HIV, HPV and Chlamydia trachomatis), as well as examples of successful research and clinical uptake stemming from biobanks. Our article also outlines key issues with respect to data privacy in infectious disease genomics, as well as the utility of adequately designed and maintained electronic health records. We maintain that the public should be able to easily access a clear and detailed outline of regulations and procedures for sample and data utilization by academic or commercial investigators, and also should be able to understand the precise roles of relevant governing bodies. This would ultimately facilitate uptake by researchers and clinics. As a result of the efforts and resources invested by several networks and consortia, there is an increasing awareness of the prospective uses of biobanks in advancing infectious disease genomic research, diagnostics and their clinical management.
\end{abstract}

Key words: biobank; infectious disease; host genomics; HIV; HPV; Chlamydia trachomatis 


\section{Introduction}

Over time, the definition of a "biobank" has moved away from an early view of a biobank as population-based to include a wider typology of biobanks that we find in the literature today. In a survey conducted in 2012, researchers involved in managing sample collections were asked about their definition of biobanks. The results of the survey showed the consensus among respondents that the term biobank may be applied to biological collections of human, animal, plant or microbial samples. Additionally, the term biobank should only be applied to sample collections with associated sample data, and to collections that are managed according to professional standards [1].

In post-Human Genome Project research, the role of biobanking as a component of research infrastructure is broadening, as knowledge from biobanks contributed to the understanding of the etiology of multifactorial diseases caused by both mutations in a variety of genes and the influence of environmental factors and lifestyle [2, 3]. Furthermore, biobanks have paved the way for the evolution of personalized medicine, especially the development of "tailored" drugs [4]. In recent years, integration, analysis and interpretation of data originating from biobanks have begun to play a growing role in our understanding of genetic susceptibilities of infectious diseases. The actuality of infectious diseases and the perpetual challenge they pose for researchers and physicians is reflected in both the high prevalence and the high mortality of existing and growing incidence of emerging infectious diseases [5]. In fact, infectious diseases represent a major health threat worldwide, and are a particularly significant burden to developing countries [6].

The importance of genetic factors in the pathogenesis of infectious diseases has transformed our understanding of such diseases by incorporating host genetic determinants that modulate immune responses as factors of pathogenesis. We now understand that host responses can determine the outcome of an infection as much as - if not more than - the properties of the pathogen itself [7]. Genomics outlined molecular biomarkers and pathways as targets for diagnosis or intervention in the field of infectious diseases [8]. Relations between genetic factors and susceptibility to the course and the outcome of infectious diseases are predominantly studied through candidate genes, genome wide associations, and twin studies [9]. This research means that biobanks (especially large 
international networks of biobanks driven by the needs of researchers, who require large collections of samples) are an imperative infrastructure for research in host genomics [10].

According to Gotweiss and Zatloukal [4], there are four main types of biobanks: clinical case/control biobanks, which contain biological samples taken from patients with specific diseases and from healthy control patients population-based biobanks, which contain samples from smaller or larger subsets of a population with or without a certain disease;

population isolate biobanks, which contain homogenous genetic material of the population represented; and

twin registries, which contain samples from monozygotic and dizygotic twins.

Biobanks contain both samples and data; this twofold nature is the root of much of the legal and ethical controversy surrounding biobanks today. Issues concerning privacy health-related information, informed consent, secondary use of samples, and harmonisation of legislation and networking of biobanks are often researched in conjunction with the term "biobanking" [11]. The potential impact of biobank-generated knowledge (and its becoming an integral part of public health policies) on our understanding of the etiology of disease, on improving diagnosis and treatment, and ultimately on the health of individuals and populations as a whole has been largely ignored thus far [3].

Uses of biobanks for public health are [2]:

- timely, responsible and effective integration of genome-based health technologies and information into health research, policy and practice;

- $\quad$ supporting the translational process from basic knowledge generated in existing biobanks to the development and implementation of health policies, interventions and programs;

- recognizing the multi-tasking nature of biobanks in the accommodation of different needs by enhancing the ability of biobanks to serve researchers and other relevant stakeholders with particular public health perspectives. 
In 1990, Lee identified what he considered to be the ideal properties of a biospecimen bank: a secure, ongoing source of funding; a cryogenic storage facility; selection criteria for obtaining and keeping the best samples in storage; and ensuring the continuation of research to optimize the collection and handling of samples [12]. De Paoli [13] also identified what he considered to be the main roles of biobanking in microbiology research:

- to enable unfettered epidemiological research: prospective use of biobanking is key to detecting and tracking different strains, comparing new strains with previously stored ones, determining modes of transmission, and ultimately combating infections

- to ensure progress in diagnostics: by comparing samples taken from the same subjects over time or by comparing samples taken from different subjects at the same point in time, or by applying novel diagnostic tools to the analysis of samples that exhibit increased sensitivity and specificity.

- to manage studies with large sample sizes: this may refer to research with sample collections coming from different geographical locations, or research that is conducted in several remote laboratories.

- to establish biorepositories with characterized host cell lines: cell lines can be used for research, diagnostics and quality control, and other scientific pursuits.

- to assist in building a microbial tree of life: such biobanks provide the basis for mapping out microbial diversity and evolution. Given the increasingly imminent threat that emerging highly virulent or therapy-resistant strains pose for global health, the importance of these types of biobank collections will likely rise in the near future.

In addition to these roles, biobanks can serve as the foundation for conducting research in host genomics and other 'omic' sciences, elucidating the role and interactions of the host's immunogenetic factors in infections [14], as well as driving prospective diagnostic and therapeutic advances [15].

This article examines the role of biobanks in the basic research in infectious disease genomics, and also observes the relevance and applicability of biobanks in both the translation of impending knowledge and the clinical uptake of biobankgenerated knowledge in infectious diseases. Our research identifies potential 
fields of interaction between infectious disease genomics and biobanks, in line with the global trend of integration of genome-based knowledge into clinical practice.

\section{Materials and Methods}

A literature search was performed on the identification of the existing links between biobanking and infectious diseases; on points of potential collaboration between biobanks, clinics and surveillance agencies; and on the examination of the relevance of electronic health records (EHR) in genomic research of infectious diseases. The study focused on examples of the translation of biobank-generated genome-based knowledge to everyday clinical practice. Databases (PubMed, Cochrane library, Google Scholar), electronic journal collections (Maastricht University EJ collection) and the websites of relevant organizations, networks and consortia (OECD, EAPM, $\mathrm{P}^{3} \mathrm{G}, \mathrm{BBMRI}$ ) were searched for appropriate references. The terms used in the searches were ["biobank*" AND ("infectious disease*" OR "genomic*")]. Retrieved articles were further selected based on relevance. Additional search terms were "public health", "data management" and "data privacy".

This article provides examples of existing biobanks with substantial resources for infectious disease research, such as those for human immunodeficiency virus (HIV), Human papillomavirus (HPV), and Chlamydia trachomatis (CT).

\section{Results}

Human versus microbial sample biobanks

There is an obvious delineation between samples taken from human individuals suffering from a pathological condition related to an infection (who may or may not be infected with or are carriers of the pathogen) and samples of the infectious agent itself. The former is primarily relevant to host 'omic' research, as it provides the material basis for both candidate gene/SNP-approaches and genome-wide association research in seeking (co)morbidity associations, and also investigates the pathogen interactions with host proteomes [16]. These samples should be paired with relevant categories of patient phenotypic data. Pathogen samples enable epidemiological studies, investigate genetic strains of that species, and develop better diagnostic tools and novel therapies [13]. 
Biomaterial samples may differ in processing and storage, as well as in shelf-life. As biomedical science moves away from the deconstruction of living systems and turns towards a more integrative, all-encompassing approach (through the likes of systems biology) [17], it is reasonable to expect that researchers increasingly begin to assess host 'omic' data together with infectious agents 'omic' profiles. As a prerequisite, adequate samples should always be accompanied by relevant data.

\section{Infectious disease genomics and the inflow of data}

Advances in sequencing technologies have resulted in a relentless influx of data that need to be interpreted. Currently, researchers are generating data more rapidly than can be analyzed. In particular, the genetic variability of bacteria accumulated through evolution is enormous and significantly increases the volume of datasets. Public health genomics specifically emphasizes the need to examine all 'omics' $[17,18]$, which, in the case of infectious disease, involves both the host and the pathogen. This emphasis dramatically increases demand for the effective deciphering of large amounts of data. There are, however, efforts among members of the microbiology community to develop strategies that would make this data manageable. For example, by grouping select loci of the pathogenic bacterial strain into schemes (the so-called geneby-gene approach) and by implementing themes schemes in conjunction with conventional (sequence-based) schemes in adequate database platforms, data that is obtained through different studies and can be used more effectively in combined analyses [19]. In this way, "genotype summaries" of selected genes could be linked to phylogenetic relationships and functional characteristics of bacteria, thereby helping researchers navigate vast bacterial genomic diversity.

\section{Existing infectious disease biobanks and networks}

The number of laboratories that are creating their own biobanks is difficult to quantify with full precision. However, the number of organizations that are developing nation-wide or transnational collections and are building large consortia and networks is ever increasing [13]. Large networks such as the Public Population Project in Genomics and Society ( $P^{3} G$ ) (http://p3q.org/) and Biobanking and Biomolecular Resources Research Infrastructure (BBMRI) (http://bbmri.eu/) are changing the landscape of international collaboration in 
biobanking, harmonizing regulatory legislation and thus facilitating the use of biobanks in research [20]. Promising nation-wide models have also emerged, including the United Kingdom Biobank (http://www.ukbiobank.ac.uk/), the Swedish National Biobank programme (now the Swedish arm of BBMRI, http:// www.bbmri.se/), the Iceland Biobank, and others. Some biobanks, however, are not positioned as public domain entities; in the case of Iceland, for example, the biobank is a private company that has been given a commercial data license [21].

This article presents several infectious disease biobanks and their effective usage, which has led to cases of successful clinical uptake (or its near-future prospects) for HIV, CT and HPV (for which there is a more substantial body of literature available). These biobanks are founded and governed by hospitals and academic institutions and provide clear examples of how biobanking can stimulate research in infectious disease genomics.

\section{HIV}

The Infectious Diseases Biobank (IDB) at King's College London is an oft-cited example of an infectious disease-oriented biobank [22, 23]. The IDB is actively collecting samples from patients infected with HIV, hepatitis B and C viruses, and invasive bacteraemias (such as the methicillin resistant Staphylococcus aureus (MRSA)). The IDB is also collecting samples from healthy control subjects. The number of HIV patients with archived materials in the IDB is steadily increasing, resulting in the availability of distinct patient cohorts (in meaningful numbers) to researchers. Data on the IDB's website indicate that by September 2010, HIV sample donations had reached 500 annually. Examples of research stemming from this collection are (as stated on the IDB website): the roles of vpu gene and tetherin in HIV/AIDS pathogenesis; gene expression signatures in in vivo and in vitro HIV-1 infection; non-infectious HIV co-morbidities; renal function and bone homeostasis in patients on HAART; the definition of CD161+ CD8+ $T$ cell subset function in HIV infection and their response to therapy; the effect of Maraviroc on microbial translocation in HIV infected individuals receiving antiretroviral therapy; and the metabolic impact of Darunavir/ritonavir maintenance monotherapy after successful viral suppression with standard Atripla in HIV-1-infected patients. Aside from archiving biological samples, the BioBank runs a database in which the following clinical information on HIV donors 
is archived: histories of CD4+ cell numbers and plasma viral loads; last known HIV negative result and first positive HIV test; birth date; ethnicity; gender; HAART administering; and other complications. The database also features sample processing information (dates and times of venepuncture, processing and freezing); and details on aliquots that have been stored or transferred to researchers.

Another example of an infectious disease-oriented biobank is the Spanish HIV BioBank [24]. The primary objective of this biobank is to further scientific knowledge about HIV infection by providing biological samples from HIVinfected patients that are included in cohorts for the objective of carrying out research. The HIV BioBank receives samples from 28 hospitals, spread across Spain, which are grouped into 6 cohorts of HIV-patients, each with defined characteristics. Any member of the AIDS Network, or any party to a relevant collaboration with a member can apply for samples. Sample release applications are evaluated by members of the Scientific Committee. If the project is approved, the researcher signs a Release Agreement with the director of the BioBank and with the coordinator of the Cohort. The BioBank and the Cohort are responsible for locating the type and number of samples needed to carry out the project. Once a year, after the samples have been released, the principal researcher sends a scientific report to the BioBank containing his or her results, such that the BioBank can maintain up-to-date records on all projects.

\section{Chlamydia trachomatis}

Partners of the EpiGenChlamydia Consortium (urogenital and ocular CT infections), coordinated by the London School of Hygiene and Tropical Medicine (by David Mabey and Robin Bailey and their Gambian partners) who are researching ocular Chlamydia-related conditions, have already defined and secured 1500 case-control pairs $(n=3000)$. More than 4000 specimens that are currently in use have been collected by Dutch partners, and 10000 specimens are available for further studies [25]. One goal was to build a biobank and data warehouse - a biomedical, ethically-developed and run central sample collection and data management system. The Consortium is investigating possibilities for conducting genetic and epidemiologic Chlamydia research with samples from existing biobanks in Northern European countries. 
The Consortium also aims to structure trans-national research to such a degree that comparative genomics and genetic epidemiology can be performed on large numbers of unrelated individuals. The most pivotal deliverables of this project were biobanking and data-warehouse building. These deliverables will allow for continuous generation of scientific knowledge on CT-host interaction genetic predisposition to CT infection, and the development of tools for early detection of this predisposition. The study of sequence variation (mainly SNPs) is a technique employed by different consortium partners to gain insight into the differences in clinical courses of infection, in order to identify genetic markers for susceptibility.

A review by Malogajski et al. [26] gives an overview of immunogenetic factors that have a demonstrable effect on human susceptibility to and the severity of CT. These immunogenetic factors are alleles (determined by specific SNPs) of pathogen recognition receptor genes. Women carrying one or a combination of these alleles are at higher risk of contracting CT, or are at significantly higher risk of developing subfertility-related complications, such as tubal pathology. The review proposes the development of novel diagnostic tools for assessing individual risk faced by CT-positive women. Currently, clinicians employ CT IgG serology when assessing these risks [27]. Due to limited sensitivity and specificity of CT serology, the predictive value of this serology is weak, and as a result, many physicians recommend that women undergo additional invasive, stressful, and costly diagnostic procedures. It is estimated that $40-45 \%$ of women undergoing laparoscopy do not have tubal pathology. Additionally, false negative serology results account for about $20 \%$ of women whose tubal pathology will not be properly and timely diagnosed [28]. The proposed tool would introduce a diagnostic approach based on a combination of two factors: a predictive SNP load; and serological markers for CT infection. On-going research aims to validate this set of SNPs and a subsequent cut-off score for diagnostic purpose. This tool would be the first to use a genetic trait in the diagnosis of infectious diseases severity in the triage of women.

\section{HPV}

Similar to the translation of CT biobank-derived data into diagnostic applications for subfertility, the translation of HPV research results should contribute to better diagnostics of cervical cancer and its pre-neoplastic stages, cervical intraepithelial neoplasia [26]. Large biobanks and patient cohorts are used to 
achieve this result. Despite the anticipated outcomes of HPV vaccination (which should lead to a drop in cervical cancer incidence in a matter of decades), the needs of generations of women who were above the age of expected exposure to HPV virus (and were therefore left out of vaccination programs) ought to be addressed. The cervical scraping cytomorphology assessment, better known as the PAP test, is routinely used throughout the world as a screening tool for cervical lesions; however, the PAP has low sensitivity. The introduction of highrisk HPV (hrHPV) assessment will increase sensitivity and ease (especially in the case of self-collected vaginal swabs) to determine a woman's risk of developing cervical cancer. Referral to a gynecologist is only needed where a woman is found to be infected by a hrHPV type. Some authors propose the development of a triage tool for high-risk HPV positive women based on methylation markers; this means that a woman should only be referred for further examination if she tested positive for one or more hrHPV types and at the same time carries a combination of methylation markers indicative of a progression of pre-neoplastic stages. This approach builds upon a number of studies that show how epigenetic alterations become increasingly present with each successive stage of cervical lesion and cervical cancer, mainly in genes that are important to the progression of cancer (e.g. tumor suppressors and cell adhesion molecules), or genes coding microRNAs, whose role is to bind to the viral nucleic sequences, thereby making them inaccessible to enzymes that are replicating or transcribing. Based on the available studies at that time, the review [26] highlighted methylation patterns in MAL and CADM 1 genes as optimal markers for the development of a potential triage test [29]. A more recent review by Litjens et al. [30] reached the same conclusion, but added $\mathrm{p} 16^{\text {INK-4a }} / \mathrm{Ki}-67$ dual immunostaining and viral integration to the proposed set of markers.

\section{Relevance of electronic health records}

The rise in usage - and usability - of electronic health records (EHRs) is a demonstrably promising catalyst in the efforts to better utilize and standardize biobanks [31]. This pertains to handling the information on the biomaterial from the large cohort studies on a plethora of diseases, including infectious diseases. 'EHR-driven genomic research' can ideally be achieved using two distinct workflows. Firstly, patients with a particular infectious disease or related sequelae could be selected from the EHR by using language processing 
tools, such as natural language processing (NLP) tools. In this case, the selected population could thereupon be recruited, either for the purpose of providing samples for genomic research, or in order to verify whether residual samples taken on previous occasions could be utilized. Secondly, EHRs can be used to broaden and advance clinical characterization by adding new relevant data to the files of those individuals whose samples are already stored in another biobank or have been used in the context of a cohort study [3 1]. Electronic systems for the automated detection of notifiable diseases have, in fact, been tested using EHRs. In past decades, the term of preference was electronic medical records (EMRs). This term has gradually been overtaken by the previously mentioned EHRs, as focus slowly expanded from the inclusion of basic clinical patient data to the provision of a more complete insight into their health background and care.

The so-called ESP (Electronic Medical Record Support for Public Health) algorithmic system, which has been tested on Chlamydia records and others, assists not only in the identification and reporting of cases of notifiable disease, but also in the advancement of public health. Prospective applications of EHRs include syndromic surveillance; clinical decision support; the construction of vaccine registries; and the assessment of areas with higher prevalence of disease [32]. The incorporation of patient genome-based information into EHRs would undoubtedly act as a major driving force for genomic medicine. It would enable the investigation of potential comorbidities of genomic associations [31], and would elucidate the ways in which such associations can individually or synergistically result in increased susceptibility to or severity of infectious disease. That said, the incorporation of patient genome information into EHRs has thus far been a daunting task, since most EHR systems are not designed to include genomic data [33]. Although the linear DNA sequence is simple by nature, the sheer volume of data and the complexity of relations among the 'functional components' of DNA are significant hurdles in attempting to devise an EHR system using this information [33, 34].

\section{Data privacy and infectious disease genomics}

While the issues of data privacy and consent exceed the parameters of this article, we will briefly lay out the state of the art in this field, as well as how legal frameworks, governance of infectious disease biobanks and the handling 
of sensitive data can affect not only patient rights, but also biomedical research generally. In 2013, during the Irish presidency of the EU Council, the European Alliance for Personalised Medicine (EAPM) hosted a conference on innovation and patient access to personalized medicine, in which experts discussed recent advances in healthcare and formulated conclusions relating to these advances [35]. In order to optimize data security and to facilitate access and consent (which would allow for re-use and secondary use of data), it was concluded that robust legal regulation of personal data in scientific research should be implemented. Moreover, cross-border transfers of data for the purposes of scientific research should be stimulated in cases where such privacy instruments have been deployed. It is important to note that, in harmonizing different systems of governance, a balance must always be struck between the stimulation of cross-border transfers of data and individual rights to privacy.

The importance of data protection cannot be underestimated, especially in the handling of samples taken from individuals who are afflicted with serious infections. Genomic discoveries concerning such infections potentially create various forms of discrimination in the context of future discovery. For example, it was discovered that African-American carriers of a polymorphism conferring a Duffy antigen-negative phenotype, DARC -46C/C, are resistant to malaria (Plasmodium vivax infection). However, subsequent research into this polymorphism also revealed that carriers have a $40 \%$ increased likelihood of becoming infected with the HIV- 1 virus [36]. In this case, contrary to the protective character of CCR5 $\triangle 32$ deletion as witnessed in European populations, the disruption of the expression of a functional receptor is a major disadvantage to the carrier. Evidently, the risks of stigmatization and discrimination arising from genome-based information (the disclosure of a patient's illness or infection status being a potential infringement of patient rights) cannot be ignored. As stated in the EAPM report conclusions [35], progress must be achieved by developing trust between researchers and the public, and by promoting the equal treatment of health research data (including genome-based information included) and the removal of silos for single-use data. Since this information is theoretically unlimited in terms of longevity, robust data protection mechanisms must be in place for periods longer than the samples' shelf life [37].

At the same time, different sets of mechanisms are needed in parallel with vigilant data protection. Genome-based research necessitates large sample 
sizes in order to arrive at more reliable results; as a result, overly-restrictive data protection policies can impede research and innovation [34]. A large number of samples is necessary to identify patient subgroups of interest. There are also calls for the provision of research data to the general public, particularly in cases where the research itself was funded using taxpayer dollars [38]. Entire human genome sequences, including those of several prominent researchers, have already been made available to the general public online (open access model). In spite of this open access, the debate over balancing the right to consent versus the right to privacy is far from resolved. The fact that fewer than 13-15 genomic locations with variable repeats (or 30-80 statistically independent SNPs) can be used to identify any one individual [39] lends perspective to requests for the deregulation of data sharing. Samples that contain a 'genomic fingerprint' in combination with data relating to the presence of serious infections pose a new threat to those safeguards that ensure participant anonymity and prevent partial treatment. Due to lack of funding, many academic institutions allow private organizations to handle their genomic databases; as a result, the protection of the rights of human participants may be at risk in any future commercial uses of data [13]. 


\section{Discussion}

The aim of this review was to explore empirical evidence on the role of biobanking in infectious disease genomics and to outline the pertinent issues in setting up and utilizing biobank materials. We note that published material that provides a detailed overview of existing infectious disease biobanks and their uses to date is lacking. In order to facilitate extensive collaboration with researchers and to ensure the continuation of research on infection, infectious disease biobanks must become more visible, and must emphasize their societal impact. Thus far, the authors have encountered underrepresentation of infectious disease biobanks in publications and insufficient information on official websites. The public should be able to easily access a clear, detailed outline of regulations and procedures for sample and data utilization by academic or commercial investigators, as well as an account of the precise roles of governing bodies. Examples of procedural transparency and extensive online visibility include the King's College Infectious Disease BioBank and the Spanish HIV BioBank [23, 24]. Appropriate regulation should precede the effective translation of biobankbased research to clinical settings; such regulation necessitates intensive efforts, so as to ensure rapid clinical uptake.

In recent years, several biobanking consortia and extensive networks have been formed, and there has been a visible increase in efforts, stakeholders' involvement and resources allocated [20]. Nevertheless, infectious disease biobanks have yet to achieve their full potential. This review does, however, provide several examples of biobanks that have successfully contributed to the translation of data to clinics and patients. In order to successfully utilize biobank information in research on infectious disease, and in order to develop 'tailored' therapies based on pharmacogenomics research, adequate representation of ethnic minorities and neglected populations in biobanking is of paramount importance. Biobanks must be constructed to account for ethnic differences in susceptibility to certain infectious diseases, which themselves have been extensively documented [40, 41]. In HIV-AIDS therapy research, for example, extrapolations of potential clinical implications of allele frequency differences between different ethnicities could significantly assist doctors when prescribing therapies. Consortium for the BioBank and Pharmacogenetics database of African populations is an example of efforts paving the way for individualised 
treatments for HIV-AIDS [42]

The Consortium's biobank of anonymous samples was used to determine baseline frequency distribution of SNPs of genes affecting drug metabolism; this usage enabled the establishment of a pharmacogenetics database. Certain information can be essential for optimizing therapeutic approaches and reducing ethnic-specific adverse reactions, such as the different drug-metabolizing capacities of particular allelic versions of enzymes (such as the CYP2B6 ${ }^{*} 6$ allele) [42]. There is an argument to be made, however, that these differences are neither inherent nor applicable to all infections. Some authors argue, in terms of decreased precision of data analysis, against blind 'social inclusivity' in biobank sampling at the potential expense of 'analytical acuity.' [43]. In countries such as the UK or the US, acting more fervently upon the two aforementioned views could lead to a reevaluation of the manner in which biobanks are governed.

EHR system designers need to be encouraged to configure these systems so as to enable the incorporation of genome-based (or 'omic'-based) information. The addition of pathogen 'omic' data to an accompanying registry should be made feasible in order to promote research in infectious diseases. Other forms of research, the clinical uptake of genome-based knowledge, and the advancement of personalized medicine can all invaluably benefit from a shifting approach to health record management. Several approaches have been proposed to this effect, and each acknowledges the unique nature of genomic data [44, 34].

The unique challenges associated with biobank-based research indicate that it is in some aspects more complex than other types of health or biomedical research. One of the main obstacles to translating biobank data into the clinical setting is confidentiality and privacy, which stem from a necessary pairing of biobank information with personal and unrelated types of health information. The protection of data obtained from samples of patients who are afflicted with serious infections is of particular importance due to the potential in such cases for discrimination. Discrimination can result both from current interpretations of data, and from future research and upcoming innovations in genomic technologies. 


\section{Conclusions}

A clear overview of the usage of existing infectious disease biobanks is lacking in present literature, and we maintain that this information should be readily accessible to the public, along with clear regulatory and procedural guidelines for utilization of samples and data. This would ultimately facilitate the currently insufficient uptake by researchers and clinics. Several biobanks have, however, already set high standards in terms of instating appropriate regulation as well as enabling successful translation into clinical setting and can therefore serve as a model to other biobanks. In recent years, efforts and resources that have been invested in biobanking networks and consortia have surged. As a result, there is a higher awareness of the multitude of ways in which biobanking can advance basic research, diagnostics and - most importantly - the clinical management of infectious disease. These advances will ensure that research in biobank-based infectious disease continues to progress. 


\section{REFERENCES}

1. Hewitt, R and Watson, P. Defining Biobank. Biopreservation and Biobanking, 2013. 11 (5): p. 309-315.

2. Brand, $A M$ and Probst-Hensch, NM. Biobanking for epidemiological research and public health. Pathobiology : journal of immunopathology, molecular and cellular biology, 2007. 74(4): p. 227-238.

3. Knoppers, BM, Zawati, MH and Kirby, ES. Sampling populations of humans across the world: ELSI issues. Annual review of genomics and human genetics, 2012. 13: p. 395-413.

4. Gottweis, $\mathrm{H}$ and Zatloukal, K. Biobank governance: trends and perspectives. Pathobiology : journal of immunopathology, molecular and cellular biology, 2007. 74(4): p. 206-211.

5. Jones, KE, Patel, NG, Levy, MA, et al. Global trends in emerging infectious diseases. Nature, 2008. 451 (7181): p. 990-993.

6. Frodsham, AJ and Hill, AV. Genetics of infectious diseases. Human molecular genetics, 2004. 13 Spec No 2: p. R187-194.

7. Peng, X, Chan, EY, Li, Y, et al. Virus-host interactions: from systems biology to translational research. Current opinion in microbiology, 2009. 12(4): p. 432-438.

8. Hill, AV. Aspects of genetic susceptibility to human infectious diseases. Annual review of genetics, 2006. 40: p. 469-486.

9. Hill, AV. Immunogenetics and genomics. Lancet, 2001. 357(9273): p. 2037-2041.

10. Meijer, I, Molas-Gallart, J and Mattsson, P. Networked research infrastructures and their governance: The case of biobanking. Science and Public Policy, 2012: p. 1-9.

11. Townend, D. The Politeness of Data Protection: Exploring a Legal Instrument to Regulate Medical Research Using Genetic Information and Biobanking2012: Universitaire Pers Maastricht.

12. Lee, RE. Environmental specimen banking. A complement to environmental monitoring. Biological trace element research, 1990. 2627: p. $321-327$.

13. De Paoli, P. Bio-banking in microbiology: from sample collection to epidemiology, diagnosis and research. FEMS microbiology reviews, 2005. 29(5): p. 897-910.

14. Ballana, E, Gonzalo, E, Grau, E, et al. Rare LEDGF/p75 genetic variants in white long-term nonprogressor HIV+ individuals. AIDS, 2012. 26(4): p. 527-528.

15. Haralambieva, IH and Poland, GA. Vaccinomics, predictive vaccinology and the future of vaccine development. Future microbiology, 2010. 5(12): p. 1757-1760.

16. Zhang, L, Zhang, X, Ma, Q, et al. Host proteome research in HIV infection. Genomics, proteomics \& bioinformatics, 2010. 8(1): p. 1-9. 
17. Khoury, MJ, Gwinn, M, Yoon, PW, et al. The continuum of translation research in genomic medicine: how can we accelerate the appropriate integration of human genome discoveries into health care and disease prevention? Genetics in medicine : official journal of the American College of Medical Genetics, 2007. 9(10): p. 665-674.

18. Mardis, ER. New strategies and emerging technologies for massively parallel sequencing: applications in medical research. Genome medicine, 2009. 1(4): p. 40.

19. Maiden, MC, van Rensburg, MJ, Bray, JE, et al. MLST revisited: the geneby-gene approach to bacterial genomics. Nature reviews. Microbiology, 2013. 11(10): p. 728-736.

20. Wichmann, HE, Kuhn, KA, Waldenberger, M, et al. Comprehensive catalog of European biobanks. Nature biotechnology, 2011 . 29(9): p. 795-797.

21. Mitchell, R. National Biobanks: Clinical Labor, Risk Production, and the Creation of Biovalue. Science, technology \& human values, 2010. 35(3): p. 330-355.

22. Kozlakidis, Z, Cason, RJ, Mant, C, et al. Ethical and Legal Considerations in Human Biobanking: Experience of the Infectious Diseases BioBank at King's College London, UK Biomedical Science, Engineering and Technology,, 2012: p. 761-778; Towie, N. London hospital launches infectious disease 'biobank'. Nature medicine, 2007. 13(6): p. 653.

23. Williams, R, Mant, $\mathrm{C}$ and Cason, J. The Infectious Diseases BioBank at King's College London: archiving samples from patients infected with HIV to facilitate translational research. Retrovirology, 2009. 6: p. 98.

24. Garcia-Merino, I, de Las Cuevas, N, Jimenez, JL, et al. The Spanish HIV BioBank: a model of cooperative HIV research. Retrovirology, 2009. 6: p. 27.

25. Morré , SA, Ouburg, S, Pena, AS, et al. The EU FP6 EpiGenChlamydia Consortium: contribution of molecular epidemiology and host-pathogen genomics to understanding Chlamydia trachomatis-related disease. Drugs of today, 2009. 45 Suppl B: p. 7-13.

26. Malogajski, J, Brankovic, I, Verweij, SP, et al. Translational potential into health care of basic genomic and genetic findings for human immunodeficiency virus, Chlamydia trachomatis, and human papilloma virus. BioMed research international, 2013. 2013: p. 892106.

27. Broeze, KA, Opmeer, BC, Coppus, SF, et al. Chlamydia antibody testing and diagnosing tubal pathology in subfertile women: an individual patient data meta-analysis. Human reproduction update, 2011. 17(3): p. 301-310.

28. Lal, JA, Malogajski, J, Verweij, SP, et al. Chlamydia trachomatis infections and subfertility: opportunities to translate host pathogen genomic data into public health. Public health genomics, 2013. 16(1-2): p. 50-61. 
29. Overmeer, RM, Louwers, JA, Meijer, CJ, et al. Combined CADM1 and MAL promoter methylation analysis to detect (pre-)malignant cervical lesions in high-risk HPV-positive women. International journal of cancer. Journal international du cancer, 2011 . 129(9): p. 2218-2225.

30. Litjens, RJ, Theelen, W, van de Pas, Y, et al. Use of the HPV MLPA assay in cervical cytology for the prediction of high grade lesions. Journal of medical virology, 2013. 85(8): p. 1386-1393.

31. Kohane, IS. Using electronic health records to drive discovery in disease genomics. Nature reviews. Genetics, 2011 . 12(6): p. 417-428.

32. Klompas, M, Lazarus, R, Daniel, J, et al. Electronic medical record support for public health (ESP): automated detection and reporting of statutory notifiable diseases to public health authorities. Advances in Disease Surveillance, 2007. 3(3): p. 1-5.

33. Kullo, IJ, Jarvik, GP, Manolio, TA, et al. Leveraging the electronic health record to implement genomic medicine. Genetics in medicine : official journal of the American College of Medical Genetics, 2013. 15(4): p. 270-271.

34. Masys, DR, Jarvik, GP, Abernethy, NF, et al. Technical desiderata for the integration of genomic data into Electronic Health Records. Journal of biomedical informatics, 2012. 45(3): p. 419-422.

35. Innovation and Patient Access to Personalised Medicine: Report from Irish Presidency Conference March 20th/21st 2013. 2013, EAPM. Available from: http://euapm.eu/wp-content/uploads/2012/07/ EAPM-REPORT-on-Innovation-and-Patient-Access-to-PersonalisedMedicine.pdf.

36. He, W, Neil, S, Kulkarni, H, et al. Duffy antigen receptor for chemokines mediates trans-infection of HIV-1 from red blood cells to target cells and affects HIV-AIDS susceptibility. Cell host \& microbe, 2008. 4(1): p. 52-62.

37. Heeney, C, Hawkins, N, de Vries, J, et al. Assessing the privacy risks of data sharing in genomics. Public health genomics, 2011. 14(1): p. 1725.

38. Church, G, Heeney, C, Hawkins, N, et al. Public access to genome-wide data: five views on balancing research with privacy and protection. PLoS genetics, 2009. 5(10): p. e 1000665.

39. Lin, Z, Owen, AB and Altman, RB. Genetics. Genomic research and human subject privacy. Science, 2004. 305(5681): p. 183.

40. Dolo, A, Modiano, D, Maiga, B, et al. Difference in susceptibility to malaria between two sympatric ethnic groups in Mali. The American Journal of Tropical Medicine and Hygiene, 2005. 72(3): p. 243-248.

41. Velez, DR, Wejse, C, Stryjewski, ME, et al. Variants in toll-like receptors 2 and 9 influence susceptibility to pulmonary tuberculosis in Caucasians, African-Americans, and West Africans. Human genetics, 2010. 127(1): p. 65-73. 
42. Matimba, A, Oluka, MN, Ebeshi, BU, et al. Establishment of a biobank and pharmacogenetics database of African populations. European journal of human genetics : EJHG, 2008. 16(7): p. 780-783.

43. Smart, A, Tutton, R, Ashcroft, R, et al. Social Inclusivity VS Analytical Acuity? A Qualitative Study of UK Researchers Regarding the Inclusion of Minority Ethnic Groups in Biobanks. Medical Law International, 2008. 9(2): p. 169-190.

44. Jing, X, Kay, S, Marley, T, et al. Incorporating personalized gene sequence variants, molecular genetics knowledge, and health knowledge into an EHR prototype based on the Continuity of Care Record standard. Journal of biomedical informatics, 2012. 45(1): p. 82-92. 

PART 2 



\section{CHAPTER 3}

Chlamydia trachomatis infections and subfertility: opportunities to translate host pathogen genomic data into Public Health

JA Lal, J Malogajski, SP Verweij, P de Boer, E Ambrosino, A Brand, S Ouburg, SA Morré.

Public Health Genomics 2013, 16: 50-61. 


\section{Abstract}

Chlamydia trachomatis (CT) infections in women can result in tubal pathology (TP). Worldwide $10-15 \%$ of all couples are subfertile, meaning they did not get pregnant after 1 year. Part of the routine subfertility diagnostics is the Chlamydia Antibody Test (CAT) to decide for laparoscopy or not in order to diagnose TP. The CAT positive and negative predictive value is such that many unneeded laparoscopies are done and many TP cases are missed. Addition of host genetic markers related to infection susceptibility and severity could potentially improve the clinical management of couples who suffer from subfertility. In the present study, the potential translational and clinical value of adding diagnostic host genetic marker profiles on the basis of infection and inflammation to the current clinical management of subfertility was investigated. This review provides an overview of the current state of the art of host genetic markers in relation to CT infection, proposes a new clinical diagnostic approach, and investigates how the Learning-Adapting-Leveling model (LAL, a public health genomic (PHG) model) can be of value and provide insight to see whether these host genetic markers can be translated into public health. This review shows that the preliminary basis of adding host genetic marker profiles to the current diagnostic procedures of subfertility is present but has to be further developed before implementation into health care can be achieved. CT infection is an example in the field of PHG with potential diagnostic to be taken up in the future in the field of subfertility diagnosis with a time line for integration to be dependent on enhanced participation of many stakeholders in the field of PHG which could be advanced through the LAL model.

Keywords: Chlamydia trachomatis, Genomics, Host genetic markers, LearningAdapting-Leveling model, Molecular diagnostics, Public health genomics, Stakeholders, Subfertility, Translation. 


\section{Introduction}

\section{Chlamydia trachomatis infection}

Chlamydia trachomatis is the most common bacterial sexually transmitted infection throughout the world. An estimated 89 million cases per year worldwide are reported. The infection is often asymptomatic resulting in patients not seeking treatment. Untreated urogenital $C$. trachomatis may give rise to late complications, including pelvic inflammatory disease, ectopic pregnancy and tubal pathology [1-4]. The clinical course of chlamydial infections is heterogeneous i.e. transmission, symptoms, clearance, and development of late complications differ per patient [5-7].

Sexually acquired $C$. trachomatis is an important public health concern for its effects on reproduction. Women who develop late complications, such as pelvic inflammatory disease or tubal pathology, suffer considerable morbidity and emotional distress, and are a socio-economic burden [8].

To prevent such outcomes, early diagnosis is important. Currently, screening for tubal pathology is performed via laparoscopy, a procedure, which is invasive and expensive, labor intensive and has a risk for surgical complications. This has resulted in extensive efforts to improve noninvasive diagnostic tests to decrease the risks of current screening methods.

Much research on bacterial components, clinical and environmental factors [9, $10]$ has been done, but no definitive correlates of late complications have been identified [1 1 ]. For a variety of infectious diseases (e.g. malaria, hepatitis and meningococcal infections), it has been shown that host genetics play a crucial role in susceptibility to and severity of disease [12-14]. To estimate the role of genetics in course of infection, twin studies are a powerful tool, and Bailey et al. [15] have shown that host genetic factors contribute almost $40 \%$ to the variation in clinical course of Chlamydia infection. These results establish the potential importance of genetic studies. Den Hartog et al. [16] showed, in a cohort of subfertile women, that single nucleotide polymorphisms (SNPs) in several pattern recognition receptors (PRRs) increase the risk of developing tubal pathology following a $C$. trachomatis infection.

The innate immune response is the first line of defense against a $C$. trachomatis infection. PRRs recognize components of the bacterium, and SNPs in these genes may affect the functionality of these PRRs and may, therefore, increase 
the risk for development of late complications. In addition, SNPs in genes coding for cytokines involved in immune responses may also be influential. Recently, our group [17] reviewed the role of SNPs in PRRs and cytokines in relation to susceptibility to a $C$. trachomatis infection.

It is important that these scientific findings are utilized in the clinic and incorporated in public health policy. The Learning-Adapting-Leveling ( $L A L$ ) model [18] is a model of translating scientific data from the lab through the market and implementing it into public health policy; it will be discussed later in this review as a possible way to assimilate new findings in clinical settings.

\section{Outline of the Article}

The overarching aim of the current review is to determine if $C$. trachomatis is one of the proof of principles in the field of public health genomics (PHG) with the potential to be taken up in the future in the field of subfertility diagnosis. Therefore, we provide an overview of the current state of the art on host genetic markers in relation to infection, propose a new clinical diagnostic approach in subfertility diagnostics based on this overview and describe how the LAL model (an integrated PHG model) can be of value to see if these host genetic markers can be translated from the lab to the market and implemented into public health.

\section{Overview of CT Host Genetic Determinants of Infection}

Several studies have shown the importance of host genetic variation on the clinical course of Chlamydia infections. This section will highlight recent findings, similar to a recent review [17], divided into detection of the pathogen Chlamydia by PRRs and the subsequent intercellular signaling by cytokines, with a focus on the innate immune system. Combined carriage of SNPs in so-called traits may exhibit a stronger influence on the course of Chlamydia infections, e.g. a reduced pathogen recognition capacity in multiple PRRs may result in higher susceptibility compared to the susceptibility when only one PRR has a reduced recognition capacity. Results for trait analyses are highlighted at the end of this section. 


\section{Pattern Recognition Receptors}

\section{Toll-Like Receptors (TLR)}

TLRs are a much investigated group of receptors. Studies have shown that TLRs are essential in the host immune system by recognizing pathogenic components (pathogen associated molecular patterns (PAMPs) and danger associated molecular patterns (DAMPs) and inducing an immune response. These receptors are present on antigen presenting cells (APC) and epithelial cells; they reside both on the cell membrane and within cells. The TLR family has been studied in relation to various infectious and autoimmune diseases with varying associations $[17,19,20]$. TLRs 2,4 and 9 are well-researched TLR family members. TLR2 and TLR4, both trans-membrane pathogen receptors, recognize chlamydial peptidoglycan (PGN) and lipopolysaccharide (LPS), respectively. TLR9 is an intracellular receptor, recognizing $\mathrm{CpG}$ islands in bacteria.

Karimi et al. [19] investigated the role of 2 SNPs in TLR2 in C. trachomatis infected women and control groups: TLR2 -16934T>A (rs4696480) and TLR2 + 2477G $>$ A (rs5743708). They did not find any association in TLR2 genotype distribution for both susceptibility to and severity of the infection. However, in haplotype analysis, they showed that haplotype TG was protective for developing tubal pathology. Laisk et al. [21] also evaluated the role of TLR2 + 2477G >A (rs5743708) in developing tubal pathology and found no association, confirming the results of the single SNP analyses of Karimi et al. [19].

Den Hartog et al. [22] studied TLR4 +896A>G (rs4986790). The genotype distribution of this SNP in subfertile women with or without $C$. trachomatis infection did not differ. However, women with this SNP and who were positive for C. trachomatis IgG and CHSP6O IgG had tubal pathology. Results of this study are highly specific, but have low sensitivity, since not all women with tubal pathology had this combination of $C$. trachomatis and cHSP6O serology, and TLR4 +896 mutation carriage. Laisk et al. [21] also investigated the role of this SNP in developing tubal pathology; they did not find any association. Taylor et al. [23] studied a different TLR4 polymorphism, rs 1927911 , and found that the mutant genotype increases the susceptibility to $C$. trachomatis infections. Similarly, they found that the TLR1 rs5743618 TT genotype increased susceptibility to Chlamydia infections [23]. 
Ouburg et al. [24] studied TLR9 SNPs in a murine model, in a cohort of Dutch Caucasian women visiting a STD outpatient clinic and a cohort of subfertile Dutch Caucasian women. The overall genotype distribution did not differ between groups. However, haplotype analyses showed, though not statistically significant, that distribution of TLR9 haplotype -1486 T (rs187084), -1237 C (rs5743836), + 1174 G (rs352139), and +2848 A (rs352140) was more frequently found in women who developed tubal pathology.

\section{C-C Chemokine Receptor Type 5 (CCR5)}

CCR5 is a chemokine receptor present on several immune cells, including monocytes, dendritic cells, microglial cells, T helper 1 cells, and macrophages. A 32-bp deletion within the CCR5 gene, CCR5 $\Delta 32$, results in premature termination of the protein, altering its function [25, 26] demonstrated that this mutation has a protective effect against developing tubal pathology when both alleles are mutated. However, these results were not confirmed in a recent study [21]. For this inconsistency, the authors suggest that the ligand of CCR5, RANTES (CCL5), binds the CCR 1 chemokine receptor as well, therefore inducing a normal response. Since they also used a different study population, they addressed the importance of group selection, and as a result this may be a reason for finding different outcomes [21].

\section{Mannose-Binding Lectin (MBL)}

$M B L$ is important in the innate immune response. It binds to various carbohydrate structures of a.o. bacteria and either directly kills the pathogen or promotes phagocytosis [27]. Studies have shown that MBL inhibits a $C$. trachomatis infection [28]. Laisk et al. [21] investigated the role of 6 polymorphisms in the MBL2 gene, coding for MBL. They found that a hyperproduction haplotype of MBL2, HYA/HYA, was a risk factor for tubal pathology independent of a $C$. trachomatis infection. They also found this association, only smaller, in C. trachomatis infected patients with tubal pathology. They suggest that hyperproduction of MBL may affect epithelial cells within the genitourinary tract, inducing tubal pathology [21]. The low-producing MBL2 genotypes are associated with tubal pathology and adverse outcome of in vitro fertilization (IVF) treatment [29]. 


\section{Human Leukocyte Antigen (HLA)}

HLA codes for the major histocompatibility complex and thus, has an important function in the immune system. The HLA system has been linked to a variety of infectious diseases and disease outcomes. In the literature, a link between HLA -DQA 1 * 0102 and HLA -DQB1 * 0602 alleles, and Chlamydia induced tubal pathology has been described [30].

Another studied receptor is the major histocompatibility complex class I chainrelated A (MICA), present on a.o. natural killer cells. When its ligand binds, activating signals for natural killer cells increase. Allele MICA * 008 had a high negative correlation with $C$. trachomatis IgG antibodies. In this study, IgG antibodies were associated with tubal pathology. This group hypothesized that MICA alleles might play an important role in the development of tubal pathology. However, in the infertile women of their study, they could not establish an association between MICA alleles and tubal pathology with or without $C$. trachomatis IgG antibodies [31].

\section{Cytokines}

A variety of cytokines have been associated with disease and disease outcomes. These cytokines have important immunoregulatory functions, and alterations in function may, therefore, influence immune responses. Several cytokines have been studied in women who developed tubal pathology. Some associations were found: IL $10-1082$ A allele together with HLA -DQA 1 * 0102 and HLA -DQB 1 * 0602 alleles; these were associated with severe tubal pathology [30, 32]. Both the TNF-a-308 A allele and the IL6 CC genotypes were found to be associated with tubal pathology: the former as a risk factor and the latter as a protective factor for tubal pathology [32].

A statistically significant association between the IFNg +874 polymorphism and chlamydial tubal pathology was not found [32], nor for IL1B +3954, IL1B -511 , and IL1RN gene polymorphisms [33]. However, a study performed in an ex vivo model showed that IL 1 , in the absence of its antagonist IL 1RA, causes destruction of the ciliated cells in the Fallopian tubes [34]. In addition, SNPs in NLRP3, associated with hypoproduction of IL $1 \beta$, is involved in tubal pathology [35]. Due to these findings, one may hypothesize that SNPs influencing IL1 functionality may affect the development of tubal pathology and the rate of severity. 
The mutant allele of the IL12B rs3212227 SNP is associated with increased susceptibility to tubal factor infertility and with a more severe progression of disease [36]. The same group also demonstrated that IL 10 and IFNG genotypes affect the lympho-proliferative responses in Chlamydia infections [37].

\section{Trait Analyses}

Den Hartog et al. [16] investigated the role of 5 SNPs in 4 genes assumed to play a role in $C$. trachomatis infection. The investigated genes were TLR4, TLR9, CD 14, and CARD 15/NOD2. The risk for development of tubal pathology doubled if a patient had 2 or more SNPs within the studied genes, compared to 1 SNP. In addition, when investigating only 1 SNP in TLR4 or CD 14 [38], no association with tubal pathology was found. Due to a small sample size, no statistical significance was observed in the trait analyses; a statistical trend, however, was observed.

Ohman et al. [37] found that the combined carriage of specific IL 10 and IFNG genotypes has an additive effect on the risk for Chlamydia infection. Atik et al. [39] demonstrated that combinations of SNPs affect the adverse that trachomatous trachiasis risk decreased 5 times with the combination of TNFA (-308A), LTA (252A), VCAM 1 (-1594C), and SCYA 11 (23T) minor allele, and the combination of TNFA (-308A), IL9 (1 13M), IL 1B (5' UTR-T), and VCAM 1 $(-1594 C)$. However, trachiasis risk increased 13.5 times with the combination of TNFA (-308G), VDR (intron G), IL4R (50V), and ICAM1 (56M) minor allele. Although these results are from ocular infections, one might hypothesize that similar effects might be observed in urogenital infections.

Although compelling, these results have to be confirmed in other studies, and additional SNPs have to be added in order to define the SNP profiles that are associated with and would help predict a patient's predisposition to Chlamydia infections and tubal pathology. This requires large cohorts which can be obtained via large consortia, in which different disciplines contribute to the overall goal.

\section{Consortium Approaches: EU Framework Program EpiGenChlamydia}

To perform large scale typing for the identification of genetic biomarkers, large and clinically well-defined cohorts are needed. Toward this end, a small consortium was founded in 2005 with Dutch, Belgian and American partners with expertise on clinical, epidemiological, bacterial, animal, immunological, 
and host immunogenetic studies to have an integrated approach to study $C$. trachomatis infections, especially the clear inter-individual differences in the clinical course of infection. This consortium was named the ICTI consortium [40, 41]. Members of the ICTI consortium applied for and obtained funding from the European Union as a large international consortium consisting of 20 partners, the EpiGenChlamydia (EGC) consortium [42].

This Chlamydia consortium was funded by the EU Framework Program 6 under the Coordination Actions in functional genomics research for a period of 2.5 years and provided its closure report in 2010. The aim of this consortium was to structure transnational research to such a degree that comparative genomics and genetic epidemiology on large numbers of unrelated individuals could be performed with future funding. This funding made it possible for 20 groups from Europe, Africa and the USA to participate (see http://www.EpigenChlamydia.eu for details). The overall goal of the EGC consortium was to accommodate the optimal environment to build and prepare a consortium to reliably determine the genetic predisposition to infection in both ocular and sexually transmitted C. trachomatis. This will allow the development of diagnostic tools that can determine an individual's predisposition to infection and the risk to develop late complications. Further, it was hoped that the knowledge generated through this effort would contribute to the understanding of the Chlamydia - host interaction, in order to allow the development of novel tools for the detection and treatment of and vaccine development for $C$. trachomatis infections.

The EGC consortium has provided the final reports to the EU including state-ofthe-art reports on the epidemiology of both ocular and sexually transmitted $C$. trachomatis infections [4], bacterial typing [43, 44], immunogenetics [17], SNP genotyping strategies, and sample validation. Two deliverables were of major importance for the future success in translation of immunogenetic markers and for obtaining new funding for a biobank, consisting of physical and virtual sample collections, and a data warehouse in which genotyping data together with clinical and demographical data is merged and accessible.

The partners working on ocular Chlamydia diseases coordinated by the London School of Hygiene and tropical medicine (David Mabey and Robin Bailey together with their Gambian partners) have already defined and secured 1,500 casecontrol pairs (total $n=3,000$ ). The scientific coordinator of the $E G C$ consortium (S. Morré) together with Dutch collaborators have collected more than 7,000 
specimens which are at present in use, while 10,000 specimens are available for further studies.

Currently, part of the consortium has obtained new funding from the EU based on Small-to-Medium-Enterprise collaborations with universities. This EuroTransBio grant has as main goal to develop a diagnostic test on the basis of human genetics and $C$. trachomatis serology to better assess the presence of C. trachomatis -associated tubal damage in subfertile women. This consortium, ending in the beginning of 2015 , is in progress of performing large scale analyses of human genetic variation to identify novel genetic markers that are able to stratify patients with tubal pathology. The identified SNPs in the PRR genes have already shown to be highly predictive for the development of tubal pathology. However, single SNPs do not provide a high enough predictive value for a diagnostic test. By combining multiple identified and novel SNPs in the PRR genes and genes in linked pathways, and exploiting them as susceptibility markers, a highly predictive test for tubal pathology-based subfertility can potentially be developed. This will be discussed further in the next section.

\section{Improvement of Subfertility Diagnostics Based on Host Genetics}

Subfertility poses an enormous burden on healthcare and society throughout the world. Worldwide, $15 \%$ of couples trying to conceive suffer from subfertility $[45,46]$. In women, one of the major causes of female subfertility is tubal pathology [45]. In tubal pathology, C. trachomatis is the single most common cause for infertility [45].

From all subfertility problems in women, tubal damage is a common cause of infertility. It includes tubal obstruction and pelvic adhesions resulting from infection, endometriosis and previous surgery. The current diagnostic procedure for diagnosing this condition can be performed by sonohysterography/ hysterosalpingo contrast sonography, hysterosalpingography(HSG), fertiloscopy, falloposcopy, or laparoscopy and dye hydrotubation, often using detection of IgG antibodies against $C$. trachomatis as first screenings tool.

Despite their effectiveness, the above-mentioned methods are costly and invasive [47] and not suitable for screening. There are several test methods available to assess the risk of $C$. trachomatis -associated tubal pathology in subfertile women. The reference standard for diagnosing tubal pathology in subfertile women is laparoscopy. However, laparoscopy has several disadvantages. First, 
it is an invasive, expensive procedure (on average 3,000 Euros, including additional costs) and requires general anesthesia. Furthermore, it holds a $1.5 \%$ risk of surgical complications (e.g. bleeding, infection).

Since it is widely recognized that a $C$. trachomatis infection is the single most common cause of tubal peritoneal damage (WHO task force on the prevention and management of infertility [48]), detecting evidence of infection using serology is noninvasive, simple and quick to perform [47]. As such, Chlamydia serology is often used as a first screening test for tubal damage in infertile women but has a limited sensitivity of $50-60 \%$.

Currently, women with subfertility are screened for a $C$. trachomatis using serology (see figure 1). Serologic testing (CAT: Chlamydia antibody testing) for C. trachomatis is based on micro immunofluorescence assays. Elevated titres of IgG are highly predictive for infection with $C$. trachomatis. These serologic assays focus mainly on the major outer membrane protein $\mathrm{A}$, which is an antigen present in the outer membrane of a chlamydial particle. When the serology outcome is negative, no further action is taken, and the couple is asked to try for one more year to get pregnant. Some women undergo HSG. If the outcome is negative (in most of the cases), they try as well to conceive for one more year. If positive (in around $5-7 \%$ of the cases) a laparoscopy will be performed, and up to $5 \%$ will have tubal pathology and will proceed to IVF procedures. Since HSG does not identify many new cases (5\% of HSG positive cases), its positive predictive value is almost identical to serology. The low specificity of the test also causes misdiagnosis because women that are negative in the serology test may in fact have tubal pathology in up to $20 \%$ of cases (percentages based on the cohort described in ref [16], personal communication). 


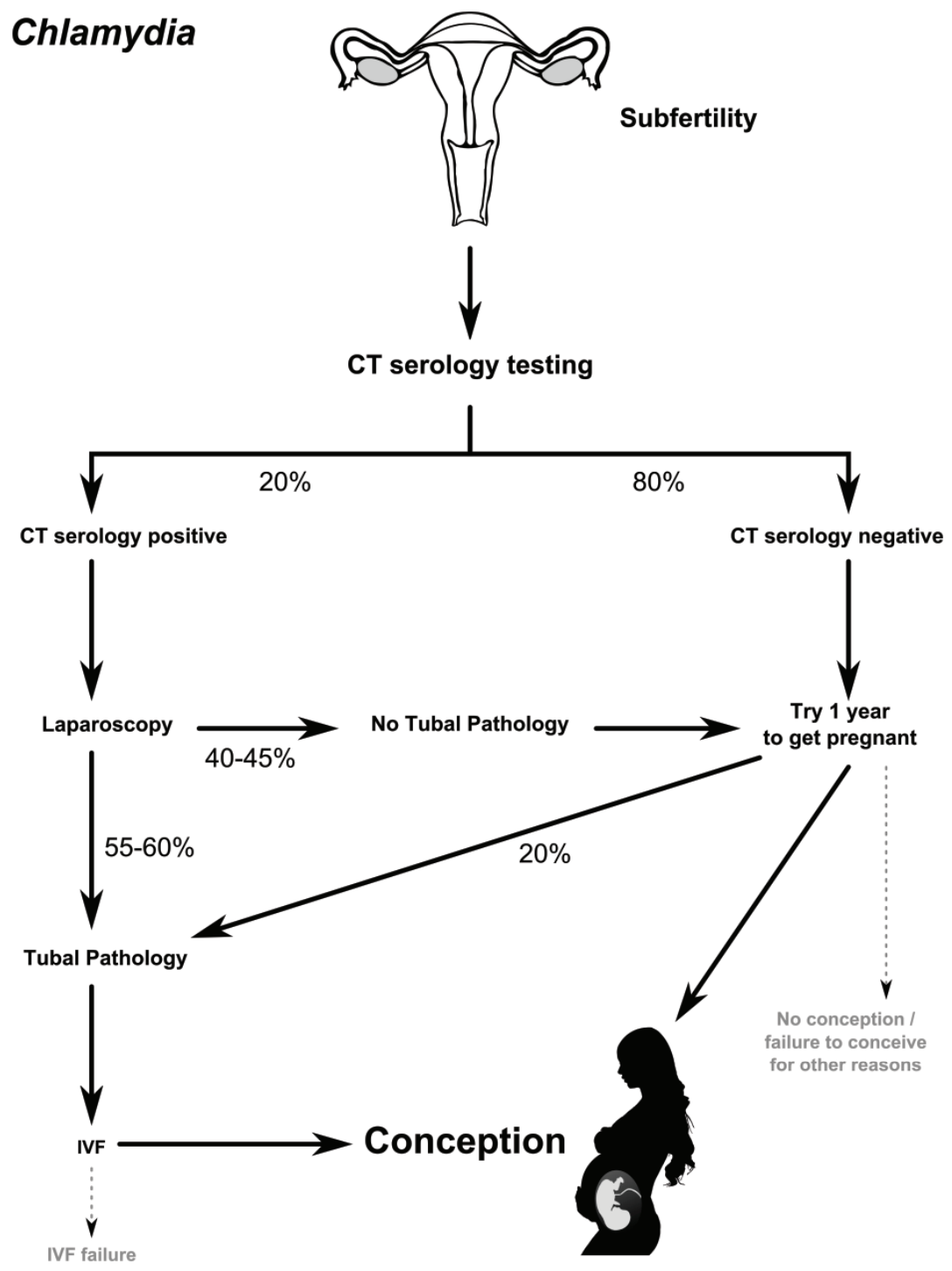

Figure 1. The relation between serology testing (Chlamydia antibody testing) and laparoscopy and the percentages of misdiagnosis. $C T=C$. trachomatis; IVF = in vitro fertilization.

If the serology outcome is positive, laparoscopy will be used for further diagnosis. When tubal damage is detected using laparoscopy, it is likely that an IVF procedure will be initiated to get pregnant. One of the drawbacks of serology is that it comes with limited sensitivity: $55-60 \%$ of the Chlamydia serology positive women actually have tubal pathology. Subsequently, this means that $40-45 \%$ of the women are serology positive and undergo laparoscopy while 
there is no tubal disease. In conclusion, in the serology negative and positive groups, a significant percentage of the women get either unneeded laparoscopies or get misdiagnosed.

Given the prevalence of subfertility, each year an estimated number of 300,000 women visit the general practice with $C$. trachomatis -associated infertility in Europe. Using the current diagnostic procedures, there is still a substantial subset of women that are misdiagnosed. This poses an enormous psychological burden on these women. Additionally, there are tremendous economic costs associated with the disease.

Despite the rapid development in the field of human reproductive medicine, there is still a medical need for diagnostic tools that are able to stratify clinically relevant $C$. trachomatis infections. The diagnostic test envisioned should be able to predict $C$. trachomatis -based tubal pathology and subsequent infertility by complementing serology in such a way that not only the sensitivity, but also the positive and negative predictive values increase significantly.

Epidemiological studies have demonstrated a $40 \%$ inheritable component for Chlamydia infections in humans [15]. This suggests the need for studies to identify which genes are responsible for this $40 \%$ component of risk. The innate immune system plays a pivotal role in the first recognition of Chlamydia and the subsequent immune response.

Since a panel of human PRRs are involved in the recognition of Chlamydia, we hypothesized and have shown that carrier traits (i.e. carrying multiple SNPs in multiple genes) result in a higher aberrant immune response as compared to single gene association studies. Subsequently, these traits synergistically increase risk for tubal pathology following $C$. trachomatis infection. Of 227 subfertile Dutch women, we performed genotyping of PRR genes TLR9, TLR4, CD 14, and CARD 15/NOD2 looking for common versus rare alleles [16]. Subfertility was defined based on laparoscopic grade of tubal pathology including extensive periadnexal adhesions and/or distal occlusion of at least one tube. Being a carrier of several rare alleles was more frequent in women with tubal pathology (who had elevated serum IgG titers against $C$. trachomatis). We showed that after $C$. trachomatis infection (these infections defined as CT IgG titers >32), subfertile women carrying $>2$ SNPs in PRR genes were at increased risk for tubal pathology compared to women carrying $<2$ SNPs ( 73 vs. $33 \%$ risk). 
This association was not found among women without IgG antibodies to $C$. trachomatis. Thus, adequate recognition of $C$. trachomatis by receptors in the genital tract is an important step in the immune response and may play a role in protecting the host against developing late sequelae following infection.

The single SNP frequencies of TLR2 SNPs did not alter the risk for tubal pathology in subfertile women, but, combined into specific haplotypes, carriage of haplotype I significantly reduced the risk of developing tubal pathology after a C. trachomatis infection ( $p=0.015, O R=0.28$ ). This haplotype also showed a significant trend in an inverse association with disease severity (asymptomatic $>$ symptomatic > tubal pathology; ptrend = 0.021) [19]. In addition, PRRs, cytokines and chemokines play an essential role in the immunopathogenesis of $C$. trachomatis infections. The chemokine receptor, CCR5, is crucial for T cell activation and function, since its deficiency causes suppression of $T$ cell responses. We showed that among patients with anti-chlamydial IgG responses, tubal pathology correlated with a low incidence of the CCR5 $\Delta 32$ deletion (7\%), while women without tubal pathology had a higher incidence of the CCR $5 \Delta 32$ deletion (31\%), as compared to controls (19\%) [26]. Thus, inflammation associated with CCR5 may predispose to development of complications of $C$. trachomatis infection. Recent findings show a similar pattern for CXCR5 [49].

Therefore, single SNPs, haplotypes, and eventually larger genetic traits based on genetic variation in multiple genes can potentially be used as susceptibility or severity markers for tubal pathology as a result of a $C$. trachomatis infection. By combining multiple SNPs in one diagnostic test, high predictive values can be achieved which should be suitable as a future companion diagnostic in the diagnosis and treatment strategy for subfertility. 


\section{Public Health Genomics Approached and Translation into Public Health}

Findings in the field of immunogenetics of $C$. trachomatis infections are of high relevance for public health and healthcare in general. Such results contribute to the understanding of infection with this agent, which is worldwide the leading cause of preventable blindness and the most prevalent sexually transmitted disease that is strongly associated with ectopic pregnancy, tubal infertility and pelvic inflammation. Furthermore, our findings provide new insights into the pathways that help explain individual heterogeneity in the clinical course of $C$. trachomatis infection and the possible development of more targeted and personalized approaches in the prevention, diagnosis and treatment of disease. To improve the health outcomes associated with $C$. trachomatis infections, there is an urgent need to timely translate immunogenetic findings into the healthcare system. The specialty having this task is called PHG, which is defined as 'the responsible and effective translation of genome-based knowledge and technologies into public policy and health services for the benefit of population health' [50]. In the context of $C$. trachomatis infections, such a public health initiative has not been advanced, despite sufficient data and the significant need to do so. However, significant public health strategies need to be implemented very early after the discovery phase, and proofs of concept need to be obtained to promote a faster translational process not only from bench to bedside, but also from bedside to healthcare. Possible bottlenecks for implementation need to be identified.

Developing diagnostic tools based on host genetic predisposition can help determine an individual's risk (as well as late complications) of infection. However, moving forward with such a plan has hurdles along the path. We have seen that C. trachomatis infections, symptoms and complications can differ between individuals on the basis of host genetic factors, ethnicity and environmental factors. And based on this review and on the literature, it seems likely that diagnostic assessment will allow for inclusion of a large number of case-specific variables (i.e. more stratified) that may even become 'truly' personalized in the near future with the incorporation information derived from dynamic fields of investigation such as systems biomedicine and epigenomics [50]. The major obstacle to implementation is not the CE (Conformtie Europeene) IVD or FDA approval of diagnostic application, but originates with the healthcare integration [18] and policy embedment processes. In general, a timely translation with 
direct implementation by the healthcare systems is low [51], which is illustrated by the large amount of data present in the literature [52] patents [53, 54] and marketed products $[55,56]$ compared to technologies being used in hospitals $[51,57]$. In order to minimize failure, it is important that researchers take into account the policy aspect and the acceptance of diagnostic applications in the healthcare systems [18] itself, instead of just considering the market.

In order to move into the healthcare systems, it is important to think from the decision-making and policy implementation perspective. Health policy is generally developed through evidence-based interventions and around general public health instruments, such as Health Technology Assessment (HTA) among others [58, 59]. We use the term Public Health Assessment Tools (PHAT) [18] when we refer collectively to Health Needs Assessment, HTA and Health Impact Assessment. Health Needs Assessment is a systematic method of reviewing the health issues facing a population, leading to agreed upon priorities and resource allocation that will improve health and reduce inequalities [59]. HTA is a multidisciplinary field of assessment that evaluates the medical, economic, social, legal, ethical, and other implications of the incremental value, diffusion and use of a technology in healthcare [60]. Health Impact Assessment is a combination of procedures, methods and tools by which a policy, program or project may be judged as to its potential effects on the health of a population and the distribution of those effects within the population. For simplicity, we will utilize HTA [61 ]. This assessment is generally done by HTA professionals in the form of systematic reviews of an emerging or new technology/tool with recommendations forwarded to decision-makers. Based on these recommendations, a technique, technology, tool, or process is implemented in the policy of healthcare, and these policy decisions determine the acceptability, the applicability in healthcare and the reimbursements. This in our observations has been generally neglected [18] by the academic-industrial complex [62]. Therefore, it is of uttermost importance to take into consideration the HTA process while developing a tool, diagnostic kit or technology, thus, in order to efficiently, effectively and in a timely manner introduce an innovation into the healthcare system. Although HTA or PHAT themselves are not sufficient when looking at the whole translational pipeline from bench to healthcare, HTA needs to be streamlined and integrated with the technology transfer process, which is the process of translating an idea into an innovative product on the market. 
In the case of $C$. trachomatis, it can be said with some certainty that HLA, specific cytokines including IL-10 and several TLRs play a role in infection and disease progression. These factors will likely vary between ethnical groups. For example, the most important TLR4 SNP, associated with tubal factor infertility in Caucasians, does not exist in Chinese people at all. Furthermore, the current needs of the population, relevant technologies on the market and prioritizing technologies based on applicability, the target audience, and stakeholder involvement including patient groups need to be taken into consideration. Also, the analytical validity (the ability of a test to accurately and reliably measure the genotype of interest [63]), clinical validity (the ability of the test to detect or predict the phenotype of interest) [63] and clinical utility (the likelihood that the test will lead to an improved outcome and incorporates assessment of the risk and benefits of genetic testing as well as economic evaluation) [63] of the diagnostic tool will have to be considered. In addition, by performing HTA, the economic, ethical, legal, and social implications will have to be thoroughly addressed in order to preempt any HTA related assessments by health policymakers for technology integration. This collectively covers many aspects of HTA investigation.

Healthcare aims to improve health of the populations and that the perspective from which the healthcare decision-maker works is based on HTA recommendations. Therefore, at the end of the day, the approval of the technology lies in its clinical utility, since the analytical validity and clinical validity have been more or less addressed. Furthermore, equal weight is given to its ethical, economic, legal, and social implications (ELSI) in society. Generally, ethical, legal and economic issues are dealt with during the development of a diagnostic tool or technology; however, the social implications may be overlooked. This can be the downfall of the tool in healthcare implementation. Also, ELSI can be limited to one perspective: the industrial and not the population based perspective. Taking into account future developments such as 'truly' personalized medicine moving from clinical utility to personal utility and the use of 'personal-genome tests' [64] , current HTA as a tool for decision-making will be challenged [65] . Current HTA evaluates a technology on the population or subpopulation, but not on an individual level, which will be the need in the era of 'truly' personalized medicine. When talking in terms of economics, reimbursement through insurance companies becomes important, which is sometimes addressed during the development of 
a technology. Financially stable patients with no need of reimbursement, early adopters or 'trendsetters' do not guarantee acceptance by decision-makers as this does not represent the majority. HTA analysis is done based on the needs of the population as a whole and not based on a few elite. Therefore, these ethical questions have to be addressed here as well. HTA professionals generally prioritize technologies listed by them and investigate one or a few of them based on what they deem relevant to the current population need, which also has to be taken into account during the development of the diagnostic tool. This can be done via comparison to what already exists on how one can get a competitive advantage based on the current population need and through consultation with patient groups. This brings in another important aspect: the involvement of stakeholders. These stakeholders represent the needs of the population and should be given preference through the development of the diagnostic tool or technology. All these several aspects should be taken into account while developing a specific diagnostic kit for $C$. trachomatis subfertility, in order to decrease the chance of failure, if not guarantee its success in healthcare implementation. As a result among other factors, there is generally a delay in uptake and wide usage of diagnostic applications and technologies in healthcare systems and hospitals. Consequentially, by the time the technology or kit reaches the healthcare system, it becomes inferior in terms of efficiency and effectiveness, given the exponential growth of newer versions of emerging technologies on the market. The latter again goes through this delaying process. Recently, a new framework or model [18] has been developed which addresses all the above mentioned issues and others not mentioned here with regard to healthcare implementation. The $\mathrm{LAL}$ model brings together for the first time 2 separate entities, namely technology transfer and PHAT in a pseudoparallel initiation. Through this, the model promotes early on involvement of all stakeholders (including academics, industry, patient groups, insurance, policy makers, doctors, HTA professionals, etc.) via public-private partnership, consultation, bilateral communication, exchange of information, feedback loops, and relevant lobbying. The model ensures that through the technology transfer pipeline, all PHAT aspects are addressed, and it also encompasses the Public Health Genomics Wheel [66] as a reference frame which demonstrates the essential tasks of Public Health for integration of genome-based technologies ensuring all possible gaps are addressed. This ensures that by the time the 
technology or diagnostic application is developed, it conforms to the standards required by decision-makers based on population needs. This decreases the likelihood of rejection as a relevant tool for healthcare. The model also puts emphasis on the value of information (how much a decision-maker is willing to pay to come to a decision) [67]. This is in terms of the end-user clarity, including ease of use, relevance to the patient and doctors, and any legal issues restricting use of the diagnostic application widely. As a consequence of the value of information, adaptations in this case to the diagnostic application can be made accordingly. Through this process, by the time the technology or diagnostic application is developed, it meets all conditions of healthcare policy, therefore, facilitating timely uptake. We believe this overarching LAL model can ease the bottleneck of a real-time uptake by hospitals as well as help industry to come to an early-on strategic decision on the new technology and thus, save on resources [18]. The LAL model seems an appropriate tool and framework for the development of the Chlamydia diagnostic kit that will ensure all issues of healthcare, as well as public health conformity and industrial requirements, are met and addressed. As a result, by the time the diagnostic kit is ready, it can be made immediately available for widespread use throughout the healthcare system. This can help industry to tap into a generally wider consumer market than traditionally accessed as well as help decrease the burden of disease that more would result from delay in the technology. It becomes obvious, that public health approaches need to adjust to these developments. Thus, PHG in the future will be quite different from PHG in the past [50].

Rapid scientific advances in genomics and its application to epigenomics, microbiomics and systems biology not only contribute to the understanding of disease mechanisms, and to the characterization of each person's unique clinical, genomic, and environmental information, but also provide the option of new promising applications in patient and human health management during the whole life-course. In fact, what was just a decade ago a distant vision for a new era of public health, in which advances from the -omic sciences would be integrated into strategies aiming at benefiting population health, is now the soon-to-be realized development of effective personalized healthcare that will be 'truly' personalized medicine. The utility of most genetic tests and biomarkers is still not evidence-based enough. In the personalized medicine setting, the traditional assessment and evaluation tools are inadequate. We clearly face the need for 
a new paradigm because as we start to understand, for example, that what we call common complex diseases might be a sum of 'rare diseases'; we move from risk factors to individual pathways or networks, and from that perspective, we move from clinical utility to personal utility [68]. However, the real paradigm shift depends on the willingness to restructure policies and on the ability to train practitioners from various professions. P4 Medicine is a future vision defined by biologist Leroy Hood, and is short for 'Predictive, Preventive, Personalized, and Participatory Medicine' [69]. The premise of P4 Medicine is that, over the next 20 years, medical practice will be revolutionized by biotechnology, to manage a person's health, instead of manage a patient's disease. Although probably not around the next corner, there is a clear urgency to prepare healthcare systems and policy-makers in advance of the inevitable.

The implementation of PHG requires increased concerted actions not only on the global (http://www.graphint.org), but also on the European level. The Public Health Genomics European Network (PHGEN II), which is funded by the General Directorate for Health and Consumer Protection (DG SANCO) (http://www. phgen.eu), initiated the National Task Forces on PHG in over 15 EU Member States. Due to these initiatives, the National Institutes of Public Health took a leading role in PHG in Finland, Belgium, the Netherlands, Croatia, Poland, or Germany. PHGEN II has developed 'European Best Practice Guidelines for Quality Assurance, Provision and Use of Genome-based Information and Technologies' [70], which will assist the European Member States. The LAL model has also been integrated in these guidelines. A major international research consortium called 'Information and Communication Technology for Future Medicine (ITFoM)' anticipates the medicine of the future, based on molecular, physiological, anatomical and environmental data from individual patients (http://www.itfom. eu). The 'ITFoM project' will create the entirely new ICT that will make it possible to make general models of human pathways, tissues, diseases, and ultimately of the human as a whole. Patient-individualized versions of ICT replica ('virtual patients') will be used to identify personalized prevention and therapy schedules and side effects of drugs [71]. The LAL model will play an important role in this project to make sure that by the time the technology is rolled out, it is adapted and conformed for real-time integration in healthcare. 


\section{Conclusions and Future Prospects}

We investigated the potential translational and clinical value of adding diagnostic host genetic marker profiles relating to infection and inflammation to the current clinical diagnosis and management of subfertility, which is based on serology and laparoscopy. It is clear from a large body of evidence that host genetic factors play a role in the susceptibility to and severity of $C$. trachomatis infection, as shown by twin studies and many candidate gene studies. To bring this current host genetic work to the next level, large scale SNP typing and SNP identification in confirmation cohorts is essential and is in progress. This work will provide insight into what type of host genetic profile can help improve subfertility diagnoses and whether the added sensitivity, specificity, and positive and negative predictive value will realize the hope that carrier traits will significantly increase the ability to predict and identify those at greatest risk of severe complications from $C$. trachomatis infection. In addition, as shown by the LAL model, stakeholders have to be informed and participate early-on in the potential implementation of these findings, a major task and challenge for the field of public health genomics which can be realized through the LAL model. 


\section{References}

1. Manavi, K. A review on infection with Chlamydia trachomatis. Best practice research. Clinical obstetrics \& gynaecology, 2006. 20(6): p. 941-951.

2. Bebear, C and de Barbeyrac, B. Genital Chlamydia trachomatis infections. Clinical microbiology and infection : the official publication of the European Society of Clinical Microbiology and Infectious Diseases, 2009. 15(1): p. 4-10.

3. Akande, V, Turner, C, Horner, P, et al. Impact of Chlamydia trachomatis in the reproductive setting: British Fertility Society Guidelines for practice. Human fertility, 2010. 13(3): p. 115-125.

4. Land, JA, Van Bergen, JE, Morré, SA, et al. Epidemiology of Chlamydia trachomatis infection in women and the cost-effectiveness of screening. Human reproduction update, 2010. 16(2): p. 189-204.

5. van Valkengoed, IG, Morré, SA, van den Brule, AJ, et al. Follow-up, treatment, and reinfection rates among asymptomatic chlamydia trachomatis cases in general practice. The British journal of general practice : the journal of the Royal College of General Practitioners, 2002. 52(481): p. 623-627.

6. Stamm, WE. Diagnosis of Chlamydia trachomatis genitourinary infections. Annals of internal medicine, 1988. 108(5): p. 710-717.

7. Morré, SA, Rozendaal, L, van Valkengoed, IG, et al. Urogenital Chlamydia trachomatis serovars in men and women with a symptomatic or asymptomatic infection: an association with clinical manifestations? Journal of clinical microbiology, 2000. 38(6): p. 2292-2296.

8. Brunham, RC and Rey-Ladino, J. Immunology of Chlamydia infection: implications for a Chlamydia trachomatis vaccine. Nature Reviews. Immunology, 2005. 5(2): p. 149-161.

9. Morré, SA, Moes, R, Van Valkengoed, I, et al. Genotyping of Chlamydia trachomatis in urine specimens will facilitate large epidemiological studies. Journal of clinical microbiology, 1998. 36(10): p. 3077-3078.

10. Molano, M, Meijer, CJ, Morré, SA, et al. Combination of PCR targeting the VD2 of omp 1 and reverse line blot analysis for typing of urogenital Chlamydia trachomatis serovars in cervical scrape specimens. Journal of clinical microbiology, 2004. 42(7): p. 2935-2939.

11. den Hartog, JE, Morré, SA and Land, JA. Chlamydia trachomatisassociated tubal factor subfertility: Immunogenetic aspects and serological screening. Human reproduction update, 2006. 12(6): p. 719-730.

12. Hill, AV. Genetic susceptibility to malaria and other infectious diseases: from the MHC to the whole genome. Parasitology, 1996. 112 Suppl: p. s75-84. 
13. Frodsham, AJ. Host genetics and the outcome of hepatitis $B$ viral infection. Transplant immunology, 2005. 14(3-4): p. 183-186.

14. Smirnova, I, Mann, N, Dols, A, et al. Assay of locus-specific genetic load implicates rare Toll-like receptor 4 mutations in meningococcal susceptibility. Proceedings of the National Academy of Sciences of the United States of America, 2003. 100(10): p. 6075-6080.

15. Bailey, RL, Natividad-Sancho, A, Fowler, A, et al. Host genetic contribution to the cellular immune response to Chlamydia trachomatis: Heritability estimate from a Gambian twin study. Drugs of today, 2009. 45 Suppl B: p. 45-50.

16. den Hartog, JE, Ouburg, S, Land, JA, et al. Do host genetic traits in the bacterial sensing system play a role in the development of Chlamydia trachomatis-associated tubal pathology in subfertile women? BMC infectious diseases, 2006. 6: p. 122.

17. Morré, SA, Karimi, O and Ouburg, S. Chlamydia trachomatis: identification of susceptibility markers for ocular and sexually transmitted infection by immunogenetics. FEMS immunology and medical microbiology, 2009. 55(2): p. 140-153.

18. Lal, JA, Schulte In den Baumen, T, Morré, SA, et al. Public health and valorization of genome-based technologies: a new model. Journal of Translational Medicine, 201 1. 9: p. 207.

19. Karimi, O, Ouburg, S, de Vries, HJ, et al. TLR2 haplotypes in the susceptibility to and severity of Chlamydia trachomatis infections in Dutch women. Drugs of today, 2009. 45 Suppl B: p. 67-74.

20. van der Paardt, M, Crusius, JB, de Koning, $M H$, et al. No evidence for involvement of the Toll-like receptor 4 (TLR4) A896G and CD14C260T polymorphisms in susceptibility to ankylosing spondylitis. Ann Rheum Dis, 2005. 64(2): p. 235-238.

21. Laisk, T, Peters, M, Saare, M, et al. Association of CCR5, TLR2, TLR4 and $\mathrm{MBL}$ genetic variations with genital tract infections and tubal factor infertility. Journal of Reproductive Immunology, 2010.87(1-2): p. 7481.

22. den Hartog, JE, Lyons, JM, Ouburg, S, et al. TLR4 in Chlamydia trachomatis infections: knockout mice, STD patients and women with tubal factor subfertility. Drugs of today, 2009. 45 Suppl B: p. 75-82.

23. Taylor, BD, Darville, T, Ferrell, RE, et al. Variants in toll-like receptor 1 and 4 genes are associated with Chlamydia trachomatis among women with pelvic inflammatory disease. The Journal of Infectious Diseases, 2012. 205(4): p. 603-609.

24. Ouburg, S, Lyons, JM, Land, JA, et al. TLR9 KO mice, haplotypes and CPG indices in Chlamydia trachomatis infection. Drugs of today, 2009. 45 Suppl B: p. 83-93. 
25. Mueller, A and Strange, PG. The chemokine receptor, CCR5. The International Journal of Biochemistry \& Cell Biology, 2004. 36(1): p. 35-38.

26. Barr, EL, Ouburg, S, Igietseme, JU, et al. Host inflammatory response and development of complications of Chlamydia trachomatis genital infection in CCR5-deficient mice and subfertile women with the CCR5delta32 gene deletion. Journal of Microbiology, Immunology and Infection, 2005. 38(4): p. 244-254.

27. Turner, MW. The role of mannose-binding lectin in health and disease. Molecular Immunology, 2003. 40(7): p. 423-429.

28. Swanson, AF, Ezekowitz, RA, Lee, A, et al. Human mannose-binding protein inhibits infection of HeLa cells by Chlamydia trachomatis. Infection and immunity, 1998. 66(4): p. 1607-1612.

29. Laisk, T, Peters, M and Salumets, A. Mannose-binding lectin genotypes: potential role in tubal damage and adverse IVF outcome. Journal of reproductive immunology, 2011 . 92(1-2): p. 62-67.

30. Kinnunen, AH, Surcel, HM, Lehtinen, M, et al. HLA DQ alleles and interleukin-10 polymorphism associated with Chlamydia trachomatisrelated tubal factor infertility: a case-control study. Human Reproduction, 2002. 17(8): p. 2073-2078.

31. Mei, B, Luo, Q, Du, K, et al. Association of MICA gene polymorphisms with Chlamydia trachomatis infection and related tubal pathology in infertile women. Human Reproduction, 2009. 24(12): p. 3090-3095.

32. Ohman, $\mathrm{H}$, Tiitinen, A, Halttunen, $\mathrm{M}$, et al. Cytokine polymorphisms and severity of tubal damage in women with Chlamydia-associated infertility. The Journal of infectious diseases, 2009. 199(9): p. 1353-1359.

33. Murillo, LS, Land, JA, Pleijster, J, et al. Interleukin-1B (IL-1B) and interleukin-1 receptor antagonist (IL-1RN) gene polymorphisms are not associated with tubal pathology and Chlamydia trachomatis-related tubal factor subfertility. Human Reproduction, 2003. 18(11): p. 23092314.

34. Hvid, M, Baczynska, A, Deleuran, B, et al. Interleukin-1 is the initiator of Fallopian tube destruction during Chlamydia trachomatis infection. Cellular microbiology, 2007. 9(12): p. 2795-2803.

35. Wang, W, Stassen, FR, Surcel, HM, et al. Analyses of polymorphisms in the inflammasome-associated NLRP3 and miRNA-146A genes in the susceptibility to and tubal pathology of Chlamydia trachomatis infection. Drugs of today, 2009. 45 Suppl B: p. 95-103.

36. Ohman, H, Bailey, R, Natividad, A, et al. Effect of IL 12 A and IL $12 B$ polymorphisms on the risk of Chlamydia trachomatis-induced tubal factor infertility and disease severity. Human Reproduction, 2012. 27(7): p. 2217-2223. 
37. Ohman, $\mathrm{H}$, Tiitinen, $\mathrm{A}$, Halttunen, $\mathrm{M}$, et al. Cytokine gene polymorphism and Chlamydia trachomatis-specific immune responses. Human immunology, 2011 . 72(3): p. 278-282.

38. Ouburg, S, Spaargaren, J, den Hartog, JE, et al. The CD 14 functional gene polymorphism $-260 \mathrm{C}>\mathrm{T}$ is not involved in either the susceptibility to Chlamydia trachomatis infection or the development of tubal pathology. BMC infectious diseases, 2005. 5: p. 114.

39. Atik, B, Skwor, TA, Kandel, RP, et al. Identification of novel single nucleotide polymorphisms in inflammatory genes as risk factors associated with trachomatous trichiasis. PloS one, 2008. 3(10): p. e3600.

40. Morré, SA, Spaargaren, J, Ossewaarde, JM, et al. Description of the ICTI consortium: an integrated approach to the study of Chlamydia trachomatis infection. Drugs of today, 2006. 42 Suppl A: p. 107-114.

41. Lyons, JM, Ouburg, S and Morré, SA. An integrated approach to Chlamydia trachomatis infection: the ICTI Consortium, an update. Drugs of today, 2009. 45 Suppl B: p. 15-23.

42. Morré, SA, Ouburg, S, Pena, AS, et al. The EU FP6 EpiGenChlamydia Consortium: contribution of molecular epidemiology and host-pathogen genomics to understanding Chlamydia trachomatis-related disease. Drugs of today, 2009. 45 Suppl B: p. 7-13.

43. Klint, M, Fuxelius, HH, Goldkuhl, RR, et al. High-resolution genotyping of Chlamydia trachomatis strains by multilocus sequence analysis. Journal of clinical microbiology, 2007. 45(5): p. 1410-1414.

44. Pedersen, LN, Herrmann, B and Moller, JK. Typing Chlamydia trachomatis: from egg yolk to nanotechnology. FEMS immunology and medical microbiology, 2009. 55(2): p. 120-130.

45. Evers, JL. Female subfertility. Lancet, 2002. 360(9327): p. 151-159.

46. Broeze, KA, Opmeer, BC, Van Geloven, N, et al. Are patient characteristics associated with the accuracy of hysterosalpingography in diagnosing tubal pathology? An individual patient data meta-analysis. Human reproduction update, 2011 . 17(3): p. 293-300.

47. Akande, VA, Hunt, LP, Cahill, DJ, et al. Tubal damage in infertile women: prediction using chlamydia serology. Human Reproduction, 2003. 18(9): p. 1841-1847.

48. Tubal infertility: serologic relationship to past chlamydial and gonococcal infection. World Health Organization Task Force on the Prevention and Management of Infertility. Sex Transm Dis, 1995. 22(2): p. 71-77.

49. Jiang, J, Karimi, O, Ouburg, S, et al. Interruption of CXCL13-CXCR5 axis increases upper genital tract pathology and activation of NKT cells following chlamydial genital infection. PloS one, 2012. 7(11): p. e47487.

50. Brand, A. Public health genomics--public health goes personalized? European journal of public health, 2011.21(1): p. 2-3. 
51. Berwick, DM. Disseminating innovations in health care. JAMA, 2003. 289(15): p. 1969-1975.

52. Peng, W. Trends in biotech literature 2009. Nature biotechnology, 2010. 28(9): p. 887.

53. Snyder, M and Cook-Deegan, B. DNA Patent Database Statistics: 2010. DNA Patent Database, 2011.

54. Catalogue of Biotechnology Patents in the Mercosur 2008. Available from: http://docs.biotecsur.org/informes/en/inventario/9 patentes ms.pdf.

55. Strength and Opportunity. The landscape of the medical technology, medical biotechnology and industrial biotechnology sectors in the UK. 2009. p. 11-12, 28, 34, 38, 40. Available from: https://www. gov.uk/government/uploads/system/uploads/attachment_data/ file/298819/bis-14-p90-strength-opportunity-2013.pdf.

56. US Biotech Market Analysis: RNCOS Industry Research Solutions. 2010.

57. Managing Technology Diffusion - Discussion Paper. 2009, Canadian Agency for Drugs and Technologies in Health p. 7-8, 10.

58. Brand, A, Brand, $\mathrm{H}$ and Schulte in den Baumen, $\mathrm{T}$. The impact of genetics and genomics on public health. European journal of human genetics : EJHG, 2008. 16(1): p. 5-13.

59. Rosenkotter, N, Vondeling, H, Blancquaert, I, et al. The contribution of health technology assessment, health needs assessment, and health impact assessment to the assessment and translation of technologies in the field of public health genomics. Public Health Genomics, 2011. 14(1): p. 43-52.

60. Battista, RN. Expanding the scientific basis of health technology assessment: a research agenda for the next decade. International journal of technology assessment in health care, 2006. 22(3): p. 275280; discussion 280-272.

61. Health Impact Assessment: main concepts and suggested approach Gothenburg consensus paper. 1999.

62. Crow, MM and Tucker, C. The American research university system as America's de facto technology policy. Science and Public Policy, 2001. 28(1): p. 2-10.

63. Haddow, J and Palomaki, G. ACCE: a model process for evaluating data on emerging genetic tests. Human Genome Epidemiology: A Scientific Foundation for Using Genetic Information to Improve Health and Prevent Disease, ed. M. J. Khoury, J. Little, and W. Burke2004, Oxford: Oxford University Press. 217-233.

64. Brand, A. Integrative genomics, personal-genome tests and personalized healthcare: the future is being built today. European journal of human genetics : EJHG, 2009. 17(8): p. 977-978. 
65. Becla, L, Lunshof, JE, Gurwitz, D, et al. Health technology assessment in the era of personalized health care. International journal of technology assessment in health care, 2011. 27(2): p. 118-126.

66. Beskow, LM, Khoury, MJ, Baker, TG, et al. The Integration of Genomics into Public Health Research, Policy and Practice in the United States. Community genetics, 2001. 4(1): p. 2-11.

67. Oestreich, T. The Value of Information Part 1 - The Framework. Journal of Management Excellence: The Value of Information, 2010(9): p. 7-10.

68. Brand, A, Knoppers, BM and Ambrosino, E. Public Health Genomics journal: adjusting the agenda to future needs. Public health genomics, 2011. 14(3): p. 125-126.

69. Hood, L. Doctor's vision of the Future of Medicine. Newsweek, June $27^{\text {th }}, 2009$.

70. Brand, A and Lal, JA. European Best Practice Guidelines for Quality Assurance, Provision and Use of Genome-based Information and Technologies: the 2012 Declaration of Rome. Drug Metabol Drug Interact, 2012. 27(3): p. 177-182.

71. Lehrach, H, Subrak, R, Boyle, P, et al. ITFoM - The IT Future of Medicine. Procedia Computer Science, 201 1. 7(0): p. 26-29. 



\section{CHAPTER 4}

Diagnosis of Chlamydia trachomatis induced tubal pathology in infertile women through genomic diagnostics - exploration of health needs

J Malogajski, JA Land, SA Morré, E Ambrosino

Submitted for publication 


\section{Abstract}

Up to $30 \%$ of infertile couples suffer from tubal factor infertility (TFI), and in approximately $45 \%$ of the cases tubal pathology can be attributed to a previous Chlamydia trachomatis infection. Based on twin research and previous findings, host immunogenetic factors have been recognized as the most important factors in influencing differences in susceptibility to, course and outcome of Chlamydia infection and thus have a major influence on the development of TFI.

In this study we explored the potential need for introducing genomic testing in the routine investigation of TFI, based on the perceived health needs of infertile women in the Netherlands and the needs of clinicians for a more accurate screening test.

We identified diagnostic strategies currently in place, defined the population that will be targeted by the new intervention and explored the ways in which the use of genomic testing could improve the investigation of TFI in the Netherlands. Our new proposed diagnostic strategy is to combine Chlamydia IgG antibody testing (CAT), the most widely used screening test so far, with genomic diagnostics early in the fertility work-up, in order to increase the accuracy of screening for TFI. We postulate that serology and genome-based diagnostics combined could have higher PPV and NPV than serology alone. Additionally, the combination of tests could contribute to optimizing the timing of additional, invasive diagnostic testing (laparoscopy) in the fertility work-up, as well as optimizing the timing of starting treatment. With the introduction of host genomic markers into the assessment of TFI, infertile women could benefit from a more accurate, yet less invasive and timelier diagnosis. 


\section{Introduction}

Infertility, the inability to conceive after unprotected intercourse for at least one year, poses a great burden on healthcare and society throughout the world. Worldwide, $10-15 \%$ of couples trying to conceive suffer from infertility $[1,2]$ and in $11-30 \%$ of these couples infertility is related to tubal pathology [1]. Persistent Chlamydia trachomatis urogenital infection (here referred as Chlamydia infection) is one of the most common causes of damage to the fallopian tubes and subsequent tubal factor infertility (TFI). The proportion of TFI attributed to Chlamydia trachomatis is estimated to be 45\% [3]. This high percentage stresses the importance of understanding the basic mechanism behind ascending Chlamydia infections and its complications and translating this knowledge into practical applications in healthcare.

Two main characteristics of Chlamydia infection make diagnosis, timely treatment and prevention of late complications very challenging. First, up to $80-90 \%$ of infections in women and up to $50 \%$ in men are asymptomatic [4, 5]. Absence of symptoms impedes antibiotic treatment and contributes to the development of complications. Second, Chlamydia infection of the lower genital tract can have very diverse courses and outcomes. It is estimated that $45 \%$ of women clear the infection within the first year without developing any complications [6], in others the bacteria causes persistent infections and sequelae, such as endometritis, salpingitis, PID (Pelvic Inflammatory Disease), ectopic pregnancy and TFI [5, 7, 8].

Twin studies [9] estimated that host genetic factors are responsible for $40 \%$ of variation in Chlamydia infection characteristics and outcome. This finding provided the scientific basis for the immunogenetic approach in the research of Chlamydia trachomatis infections.

A previous study reviewing the translational potential of genomic and genetic findings related to Chlamydia trachomatis in healthcare [10] pointed out a growing body of research on host immunogenetic factors behind the differences in disease outcomes [11-16]. Such immunogenetic factors consist of genetic variations, as Single Nucleotide Polymorphisms (SNPs), in genes responsible for, amongst others, Pattern Recognition Receptors (PRR) [1 1 ]. Toll Like Receptors (TLRs) are a family of PRR with the ability to identify pathogens and to initiate an innate immune response. TLRs have been recognized as the most important 
factors in influencing differences in susceptibility to, course and outcome of Chlamydia infection [17]. Thus, research on host immunogenetic factors could have implications on the future of diagnosing Chlamydia trachomatis -induced infertility.

Chlamydia IgG antibody testing (CAT) in serum is the most commonly used test in the screening of TFI. There are a number of studies estimating the accuracy of this screening strategy [18-20]. An early meta-analysis on the predictive value of CAT [18] found that the sensitivity of evaluating Chlamydia trachomatis titers in blood widely ranges from 21-90\%, but it has been shown that test accuracy depends on the type of the essay used, definition of TFI and on the reference test applied for diagnosing tubal pathology [19]. Sensitivity for tubal pathology of the most accurate CAT was assessed at approximately $60 \%$, with specificity of $85-90 \%$ [21].

The most important research priorities related to Chlamydia trachomatis are considered to be: correlating host genetics with infection outcomes and determining the risk and timing of development of tubal inflammation and damage from untreated chlamydial infection, by developing standardized algorithms for measuring PID, ectopic pregnancy and TFI on a population level [22].

SNPs-based analysis has the potential to improve the accuracy of diagnosing TFI and such a non-invasive genomic test is currently under development [14, 23]. By identifying and combining multiple SNPs in PRR genes and their linked pathways, a diagnostic test of high sensitivity, specificity and negative predictive value (NPV) can potentially be developed [23].

The clinical relevance of a screening test based on genomic markers would be in adequately assessing the risk of TFI in infertile patients, and postponing or completely avoiding the use of expensive and invasive diagnostic procedures in patients at low risk of having tubal pathology, and selecting patients at high risk in whom laparoscopy is advised.

Today, many gene-disease associations are proven and genetic/genomic testing has acquired a role in guiding clinical decision making. However, the translation of scientific discoveries into every day clinical practice is still a slow and difficult process. It is estimated that only about $5 \%$ of discoveries in basic science are licensed into healthcare [24], and it may be assumed that translation of genomic and genetic findings is even lower. The aim of this study is to facilitate 
such translation process from bench to bedside by exploring the potential need for introducing genomic testing in the routine investigation of TFI by assessing health needs of the target population.

\section{Material and Methods}

There is no common understanding of the concept of health needs. They have different meaning for different stakeholders among healthcare providers, policymakers and patients [25]. In order to explore the possibility of improving the diagnostic accuracy of testing for Chlamydia trachomatis -induced infertility in women using genomic diagnostics, we designed a framework for assessment of health needs based on the National Health Service (NHS) approach [26]. The following 3-step analytic framework was developed Step 1 - Identify services/strategies currently in place in fertility care in the Netherlands;

Step 2- Assess the health needs of infertile women in the Netherlands and/or clinicians' needs

Step 3-Define the ways in which the use of genomic testing could improve the services/strategies currently in place and ameliorate the health outcomes of infertile women in the Netherlands.

\section{Data collection}

In order to conduct such a study, we combined data collected by literature review with data obtained by interviewing gynaecologists, selected for their expertise in reproductive medicine in 4 out of 8 academic hospitals in the Netherlands (these hospitals will later be referred to by using numbers 1-4).

The literature search focused on identifying the current strategies in the fertility work-up, concentrating on tubal infertility, and on defining the population of infertile women in the Netherlands. The following databases were searched for appropriate references: (PubMed, Cochrane library), Electronic Journals collections (Maastricht University EJ collection) and websites (Centraal Bureau voor de Statistiek website and Dutch Society for Obstetrics and Gynaecology (NVOG) website).

The purpose of the interviews was to explore the topics of fertility investigation as well as to corroborate the information gained from the literature search. The 
interviews took place between February and March 2013. They were semistructured interviews, that is, more similar to conversation then to formal structured interviews. Before conducting the interviews we created some guidelines and decided on the main issues for discussion, but we did not insist on always following the same sequence of the questions or the exact formulation used in the guidelines. The focus in the interviews was on fertility work-up in general, with emphasis on TFI work up, on the type and sequence of diagnostic procedures in routine work-up in one's own clinic and on the main perceived problems in everyday practice. Three interviews were conducted face-to-face and one via Skype. All the interviews were recorded and later transcribed.

\section{Results}

Step 1: Services currently in place

Currently, the investigation and management of infertility in the Netherlands is based on the guidelines developed by the working group of the Dutch College of General Practitioners (NHG) and the Dutch Society for Obstetrics and Gynaecology (NVOG). The guidelines emphasize the importance of shared care between gynaecologists and general practitioners (GPs) and the need for consensus among professionals in the clinical decision making process. The document provides a general outline of the recommended diagnostic procedures, leaving the final decision for the treatment and management of infertility to the GPs and specialists working at the regional level [27]. In the Netherlands, at the moment, there is no consensus on the sequence of diagnostic procedures in routine fertility work-up, including investigation of tubal pathology as a possible cause. As a consequence, diagnostic strategies in Dutch hospitals may differ considerably.

In hospital 1 the investigation of tubal function begins with CAT. Whenever CAT is negative (CAT-), the patient will undergo Hysterosalpingography (HSG). In case of abnormal HSG the patient is referred to laparoscopy. In case that CAT is positive $(\mathrm{CAT}+)$, the age of the patient will define what the following step is. CAT+ women younger than 39 undergo laparoscopy, whereas women older than 39 have no additional testing and are referred for IVF. (Gynaecologist \# 1 , 13.02. 2013, personal communication). 
In hospital 2 CAT is rarely performed. In most cases HSG is done as the primary investigation of tubal patency and laparoscopy is performed only in patients with abnormal HSG results (Gynaecologist \#2, 12.03.2013, personal communication).

In hospital 3, the patients are first screened by CAT. In case of a positive CAT, a patient is assumed to have $50 \%$ chance of either tubal blockage or adhesions, and laparoscopy is advised. In case of negative CAT, no laparoscopy is performed. In this hospital HSG as a screening test is totally abandoned (Gynaecologist\#3, 12.03.2013, personal communication).

In hospital 4 all three screening strategies (CAT, HSG and laparoscopy) are used. The screening process starts with CAT. Patients with positive CAT results undergo laparoscopy regardless of their age, patients with negative CAT results have HSG and, in case of an abnormal HSG they undergo laparoscopy (Gynaecologist \#4 14.02.2013, personal communication).
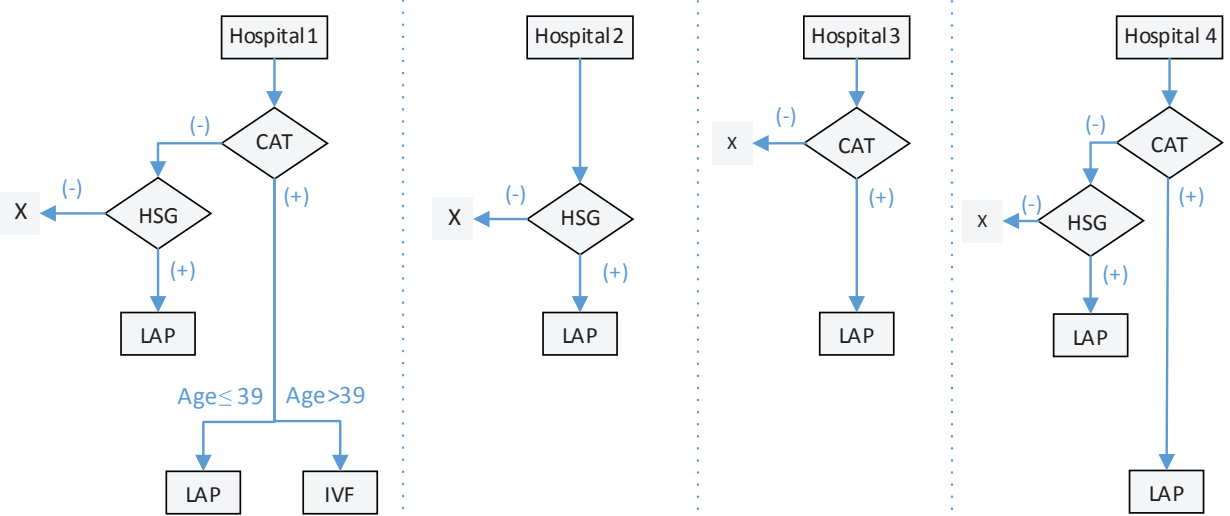

Figure 1. Schematic representation of different diagnostic protocols in different Dutch academic hospitals (HSG- hysterosalpingography; LAP- laparoscopy, CAT-Chlamydia antibody testing; IVF-in vitro fertilization; $X$ - further investigation of tubal factors concluded)

An updated and more comprehensive version of the guidelines for fertility work-up in the Netherlands is underway, and it will be based on the guidelines on infertility developed in UK by the National Institute for Health and Clinical Excellence (NICE) [28]. 
The NICE guidelines recommend HSG as the strategy of first choice for the screening of tubal pathology in the absence of known comorbidities, such as pelvic inflammatory disease (PID), endometriosis or ectopic pregnancy. Conversely, women with known comorbidities should undergo laparoscopy, which is considered the golden standard for the assessment of tubal function [28].

Step 2: Defining the target population and its health needs

Establishing health needs for the introduction of a new diagnostic strategy starts with defining the population that will be targeted and may benefit from the new intervention. There are several challenges in defining the population of women in the Netherlands who could potentially benefit from introducing genetic testing into routine clinical investigation of TFI. The exact incidence and prevalence of Chlamydia infection [29] are unknown, and there is uncertainty about the proportion of women with Chlamydia infection who will over time develop PID and TFI [20]. Therefore, in understanding our target population we have to rely on estimates and trends of Chlamydia infection prevalence found in the literature.

In the Netherlands there is no nationwide Chlamydia trachomatis screening, nevertheless in the last 10 years several rounds of screening were conducted offering an insight into the overall prevalence of Chlamydia infection. In 2003 a pilot screening project for Chlamydia infection involving 21000 participants found a $2 \%$ overall prevalence of Chlamydia infection in women and 1,5\% in men [30]. Following the pilot, a systematic and selective (initiated in three regions) Chlamydia screening program, aiming at reducing the prevalence of the infection by screen and treat, started in 2008 and lasted till 2011. In the first screening round an overall positivity rate of $4.2 \%$ was found among $15-29$ years old respondents from Amsterdam, Rotterdam and South Limburg [31]. A follow-up study evaluating the effectiveness of three yearly chlamydia screening rounds [32] did not find a significant decrease in positivity rates in any region or socio-demographic group.

The surveillance data of the Dutch National Institute for Public Health and Environment (RIVM) show that in the period from 2004 till 2012, 10-13\% of those visiting the Dutch regional STI clinics tested positive for Chlamydia. The testing was voluntary, provided free of charge and anonymous, but all the 
positive cases were reported to the National Centre for Infectious Disease Control at the RIVM. In 2012, with 15.000 diagnoses, Chlamydia became the most common bacterial cause of infection in the registries of the Dutch STI clinics. Of women who tested positive for Chlamydia $77 \%$ were younger than 25 years. [33]. However, the lack of clear guidelines regarding anatomic sites to be tested for Chlamydia (routinely only the urogenital area is tested) may underestimate the exact burden of Chlamydia infection [34]. The general trend in Europe, based on the data of reported incidence of Chlamydial infections, show increasing trends in the last ten years. This increase in positivity rate is partly attributed to the increased testing and partly to the increase in risk taking behavior [20].

However, even if exact numbers of prevalence and incidence of the infection are known this would not allow to determine the exact number of women with Chlamydia induced TFI. It is estimated that almost half of all infected women will spontaneously clear the infection [6]. There is a lack of prospective studies quantifying the risk of developing TFI over time after an untreated $C$. trachomatis infection [29]. Research offers diversified findings with PID occurring in 2-4.5\% to $30 \%$ in women with a previous untreated Chlamydia infection, and TFI to develop $10-20 \%$ of them. [29] and from studies using models the estimated risk to develop TFI after lower genital tract Chlamydia infections is 0.1-5\% [21]. Data on the actual prevalence of infertility in the Netherlands are scarce. In a study published in 1995 estimating the prevalence of infertility in South Limburg, researchers found that between 14-16.5\% of couples visited a fertility specialist during their reproductive life [35]. In an international study on the prevalence of infertility and the estimate of the demand for fertility medical care, Boivin et al. [2] found the prevalence of female infertility in high income countries to be ranging from 3.5-16.7\% and from $6.9-9 \%$ in middle income countries. Their analysis of population surveys in 25 countries found an overall median prevalence of infertility of $9 \%$, estimating worldwide more than 72 million women being infertile. In a survey in 1997, Balen et al. reported a prevalence of infertility among Dutch couples of $10.7 \%$, with more than $80 \%$ of infertile couples seeking medical care [36].

A very important characteristic of the target population is age, as female age is the single most important determinant of a couple's fertility [37]. A study conducted in the Netherlands found a strong relation between later conception 
and the increased demand for fertility care [38].

In the Netherlands, alike other high income countries, there is a trend of postponing having children. The mean maternal age at delivery of the first child has increased from 25.6 years in 1980 to 29.4 in 2008. [39, 40]. In 2012 $20 \%$ of all births were to first time mothers older than 35 [41]. In 2009, 4\% of children in the Netherlands were born to mothers who were 40 years or older [42]. In some fertility clinics in the Netherlands, the proportion of women older than 35 years seeking medical care has increased four times in the last 20 years [38].

In summary, Dutch women are postponing having children, consequently the population of women undergoing fertility investigation in the Netherlands are growing older, increasing their demand for fertility care. Data show a 5-10\% annual increase in demand for assisted reproduction techniques in many developed countries over the last 5 years [43].

Screening and surveillance data on Chlamydia infection show either persistent or increasing prevalence rates of the infection among young adults. This might have clinical implications 10 to 15 years later when these women decide to have children, resulting in increased numbers of women visiting fertility clinics and applying for assessing the risk of TFI.

Step 3: Potential of genomic testing in meeting the health needs

\section{Description of the strategy}

Our new proposed screening strategy would combine CAT with genomic diagnostics early in the fertility work-up by taking blood samples for CAT and host genomic biomarkers (SNPs). Positive CAT is considered a serological proof of previous Chlamydia infection but does not inform us about the course of infection and probability of tubal pathology [44]. The genome-based companion diagnostic, testing for variations in genes encoding for amongst others PRRs and inflammatory pathways including tissue scarring, could give us more insight in the course of infection. The test, based on combination of SNPs is under development and not yet clinically validated, based on the prior research in the following text we hypothesize its diagnostic value.

Since tubal pathology is a multifactorial and polygenic disease, and one single SNP usually does not predict a major risk, we are studying combinations of SNPs 
in traits. Based on the effects of specific SNPs there are potentially two different genetic traits that can be defined, i.e. "protecting" and "risk" traits respectively for the development of tubal pathology following Chlamydia trachomatis infection, but also after other (bacterial) STDs leading to tubal pathology. [14]. These different genetic marker traits need to be combined in a way that provides an accurate assessment of ratio between risk factors and protective traits.

The intended added value of the genome-based companion diagnostic (CAT and host genomic markers) would be to improve PPV by decreasing the number of false positive CAT results and the number of invasive diagnostic procedures performed in CAT + women without TFI. Improving NPV would reduce the number of patients with false negative tests in whom diagnosing TFI would be delayed by postponing or omitting laparoscopy.

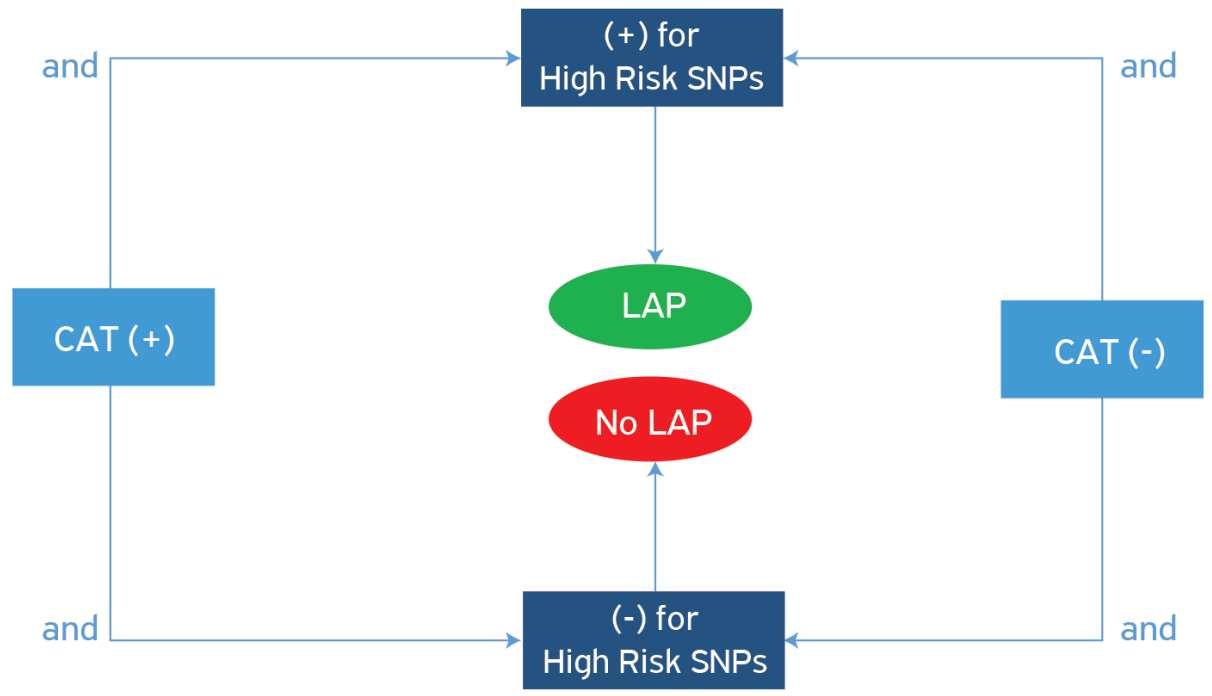

Figure 2. Elements of the decision making process based on the implementation of the "CAT and genome-based companion diagnostics" in the initial phases of the fertility work-up (HSGhysterosalpingography; LAP- laparoscopy, CAT-Chlamydia antibody testing)

Positive CAT and negative genomic markers for high TFI-risk would indicate that, although the patient had a Chlamydia infection in the past, there is a high chance of the infection to have been cleared without complications. In this case, no laparoscopy would be indicated to exclude tubal pathology. In case both tests are negative the risk of TFI is low, and further investigation of tubal function 
could be omitted. The combination of positive CAT and positive genomic markers for high risk would identify women at the highest risk of tubal pathology, and laparoscopy would be advised. Lastly, when CAT is negative and the genetic test is positive, further investigation of tubal function would be advised based on the predictive value of host genetic markers for TFI, as TFI can be caused by bacterial STDs other than Chlamydia. (Figure 2). In those cases in which the genomic test is at the same time positive for the SNPs which put the patient at high risk of developing TFI, and positive for the SNPs recognized for their protective role, the decision making will be more complex and will have to rely on additional clinical variables.

CAT and genome-based companion diagnostics are both noninvasive tests for which a small quantity of blood is needed, allowing the test to be performed in a GP office. In case of a positive test panel result, a GP could refer couples who wish to become pregnant to a reproductive specialist regardless of other clinical variables. In case of a negative result timing of referral to a specialist could depend on other prognostic factors in the couple. In the fertility clinic, combining CAT and genome-based companion diagnostics in the initial phases of the infertility investigation would enable to assess the risk of tubal pathology at an early stage. This would affect decision making on subsequent investigations and treatment (Figure 2). 


\section{Discussion and conclusion}

Today in many fertility clinics in The Netherlands, in the investigation of TFI, CAT and/or HSG are used as the primary tests, and laparoscopy as the reference diagnostic. The advantage of CAT is that it is easy to perform, inexpensive, noninvasive and not associated with complications. The main disadvantage is the relatively low PPV. Research shows that in approximately $40-50 \%$ of women who are testing positive for CAT, no tubal pathology is found at laparoscopy, whereas $10-20 \%$ of women testing CAT negative do have tubal pathology [10, $17,19]$.

In the Netherlands, HSG is either performed following CAT or as an independent test of tubal patency. HSG's sensitivity and specificity are estimated at $53 \%$ and $87 \%$ respectively (compared to laparoscopy) $[45,46]$. The main disadvantage of HSG is, apart from its mediocre test characteristics, is that is a painful procedure and can be complicated by an ascending infection.

Laparoscopy is considered the golden standard in the assessment of tubal patency, and it also visualizes endometriosis and peritubal adhesions. However, it is an invasive procedure associated with discomfort for the patient, has to be performed under general anesthesia, and is associated with potential surgical complications, a post-surgical recovery period and high costs [47].

The ultimate goal is to improve the accuracy of first line testing by correlating host genetics with infection outcomes, i.e. by combining serologic and genetic testing, to more accurately determine the risk of TFI and to reduce the number of misdiagnoses. In defining the target population that could benefit from introducing genetic testing into routine fertility work-up, we face several challenges. The greatest challenge of them, and the fundamental challenge of Chlamydia research, is not knowing the true incidence and prevalence of Chlamydia infections and the risk of developing TFI over time after an untreated C. trachomatis infection [29]. Additionally, in the Netherlands there are no recent data on the prevalence of infertility. A study conducted in 1997 [36] assessed that $10.7 \%$ of Dutch couples have problems in conceiving, with more than $80 \%$ of them seeking medical care. More recent data of the prevalence of infertility in the Netherlands are needed as since 1997 the mean maternal age at delivery of the first child has significantly increased [39], influencing female fertility and increasing the demand for fertility care. Finally, screening and surveillance data 
from STI clinics in The Netherlands for Chlamydia infection show no decrease in prevalence rates of infection among young adults, and therefore it can be assumed that Chlamydia trachomatis will continuously play an important role in the pathophysiology of TFI.

\section{Clinical relevance}

CAT has been introduced into the fertility work-up as a simple and inexpensive screening test for the risk assessment of TFI. The clinical performance of CAT, however, has been shown not to be optimal due to the number of false positive and false negative test results. [48]. The primary goal of introducing genomic tests to CAT as a combined test panel, is to improve test accuracy. This would lead to improved clinical decision making for gynaecologists, more efficient use of resources and less expenses for the healthcare system, and minimisation of interventions for the patients.

There are also challenges to be considered when proposing the introduction of genomic testing in the routine fertility work-up. One of them is complexity of the potential implementation of the new strategy. Some patients may be positive for SNPs which increase risk to develop TFI and at the same time be positive for SNPs of preventive genetic trait, making clinicians' decision process more complex and calling for precise algorithms to aid decision making. The presence of SNPs associated with risk of TFI and SNPs associated with protection against TFI could create a grey zone. This grey area should be as small as possible, but it will always exist.

Healthcare spending in the Netherlands is steadily growing, and in 2012 it equaled to 11.9 percent of gross domestic product (GDP), which is higher than the average 9.6 percent of OECD countries [42]. Growing costs of healthcare are putting emphasis on the importance of cost effectiveness of the new proposed diagnostic and treatment strategies. Therefore there is a need for a comprehensive analysis comparing costs and outcomes of the course of action proposed in our study and the existing combinations of diagnostic strategies (CAT, HSG and Laparoscopy) currently performed in the Netherlands.

Finally, advancements in genomics are driving changes in diagnostic and treatment strategies in many fields of medicine. One of the challenges for the healthcare professionals will be to adjust to the new developments. In the case of introduction of genomic testing in routine fertility work-up this could be 
achieved by setting up continuing medical education courses for reproductive specialists in order to increase their familiarity with clinical genetics. Another challenge is creating a test with straightforward results, easy to interpret and explain to patients. Such a test could contribute to the acceptance of genomic markers in the routine fertility work-up by the medical professionals.

One of the key elements for implementation of genomic applications in clinical care is its proof of its applicability and accuracy [49]. Therefore, for successful implementation of genome-based companion diagnostics in the investigation of TFI, validation of the genomic test is the most important step. Clinical studies need to be performed in order to demonstrate the benefits of the proposed strategy and to confirm that the introduction of the host genomic markers as an addition to CAT in testing of TFI will result in better patient management and improved clinical outcomes. 


\section{REFERENCES}

1. $\quad$ Evers, JL. Female subfertility. Lancet, 2002. 360(9327): p. 151-159.

2. Boivin, J, Bunting, L, Collins, JA, et al. International estimates of infertility prevalence and treatment-seeking: potential need and demand for infertility medical care. Human reproduction, 2007. 22(6): p. 15061512.

3. Price, MJ, Ades, AE, Welton, NJ, et al. How much tubal factor infertility is caused by Chlamydia? Estimates based on serological evidence corrected for sensitivity and specificity. Sexually transmitted diseases, 2012. 39(8): p. 608-613.

4. Lyons, JM, Ouburg, S and Morré, SA. An integrated approach to Chlamydia trachomatis infection: the ICTI Consortium, an update. Drugs of today, 2009. 45 Suppl B: p. 15-23.

5. Lanjouw, E, Ossewarde, JM, Stary, A, et al. 2010 European guideline for the management of Chlamydia trachomatis infections International Journal of STD \& AIDS, 2010. 21: p. 729-737.

6. Morré, SA, van den Brule, AJ, Rozendaal, L, et al. The natural course of asymptomatic Chlamydia trachomatis infections: $45 \%$ clearance and no development of clinical PID after one-year follow-up. International Journal of STD \& AIDS, 2002. 13 Suppl 2: p. 12-18.

7. Paavonen, $J$ and Eggert-Kruse, W. Chlamydia trachomatis: impact on human reproduction. Human reproduction update, 1999. 5(5): p. 433447.

8. WHO. Sexually transmitted infections. 2013; Available from: http://www. who.int/mediacentre/factsheets/fs 110/en/.

9. Bailey, RL, Natividad-Sancho, A, Fowler, A, et al. Host genetic contribution to the cellular immune response to Chlamydia trachomatis: Heritability estimate from a Gambian twin study. Drugs of today, 2009. 45 Suppl B: p. 45-50.

10. Malogajski, J, Brankovic, I, Verweij, SP, et al. Translational potential into health care of basic genomic and genetic findings for human immunodeficiency virus, Chlamydia trachomatis, and human papilloma virus. BioMed research international, 2013. 2013: p. 892106.

11. den Hartog, JE, Ouburg, S, Land, JA, et al. Do host genetic traits in the bacterial sensing system play a role in the development of Chlamydia trachomatis-associated tubal pathology in subfertile women? BMC infectious diseases, 2006. 6: p. 122.

12. Darville, T, O’Neill, JM, Andrews, CW, Jr., et al. Toll-like receptor-2, but not Toll-like receptor-4, is essential for development of oviduct pathology in chlamydial genital tract infection. Journal of immunology, 2003. 171(11): p. 6187-6197. 
13. Morré, SA, Murillo, LS, Bruggeman, CA, et al. The role that the functional Asp299Gly polymorphism in the toll-like receptor-4 gene plays in susceptibility to Chlamydia trachomatis-associated tubal infertility. The Journal of infectious diseases, 2003. 187(2): p. 341-342; author reply 342-343.

14. Morré, SA, Karimi, $O$ and Ouburg, S. Chlamydia trachomatis: identification of susceptibility markers for ocular and sexually transmitted infection by immunogenetics. Fems Immunology And Medical Microbiology, 2009. 55(2): p. 140-153.

15. den Hartog, JE, Lyons, JM, Ouburg, S, et al. TLR4 in Chlamydia trachomatis infections: knockout mice, STD patients and women with tubal factor subfertility. Drugs of today, 2009. 45 Suppl B: p. 75-82.

16. Laisk, T, Peters, M, Saare, M, et al. Association of CCR5, TLR2, TLR4 and $M B L$ genetic variations with genital tract infections and tubal factor infertility. Journal of reproductive immunology, 2010. 87(1-2): p. 7481.

17. den Hartog, JE, Morré, SA and Land, JA. Chlamydia trachomatisassociated tubal factor subfertility: Immunogenetic aspects and serological screening. Human reproduction update, 2006. 12(6): p. 719-730.

18. Mol, BW, Dijkman, B, Wertheim, P, et al. The accuracy of serum chlamydial antibodies in the diagnosis of tubal pathology: a meta-analysis. Fertility and sterility, 1997.67(6): p. 1031-1037.

19. Land, JA, Gijsen, AP, Kessels, AG, et al. Performance of five serological chlamydia antibody tests in subfertile women. Human reproduction, 2003. 18(12): p. 2621-2627.

20. Akande, V, Turner, C, Horner, P, et al. Impact of Chlamydia trachomatis in the reproductive setting: British Fertility Society Guidelines for practice. Human fertility, 2010. 13(3): p. 115-125.

21. Land, JA, Van Bergen, JE, Morré, SA, et al. Epidemiology of Chlamydia trachomatis infection in women and the cost-effectiveness of screening. Human reproduction update, 2010. 16(2): p. 189-204.

22. Gottlieb, SL, Martin, DH, Xu, F, et al. Summary: The natural history and immunobiology of Chlamydia trachomatis genital infection and implications for Chlamydia control. The Journal of infectious diseases, 2010. 201 Suppl 2: p. S190-204.

23. Lal, JA, Malogajski, J, Verweij, SP, et al. Chlamydia trachomatis infections and subfertility: opportunities to translate host pathogen genomic data into public health. Public Health Genomics, 2013. 16(1-2): p. 50-61.

24. Khoury, MJ, Gwinn, M, Yoon, PW, et al. The continuum of translation research in genomic medicine: how can we accelerate the appropriate integration of human genome discoveries into health care and disease prevention? Genetics in medicine : official journal of the American College of Medical Genetics, 2007. 9(10): p. 665-674. 
25. Wright, J, Williams, R and Wilkinson, JR. Development and importance of health needs assessment. BMJ, 1998. 316(7140): p. 1310-1313.

26. Health Needs Assessment Workshop. in National Health Service2010, National Health Service. Available from: http://cehi.org.uk/Health\%20 Needs\%20Assessment\%20Workshop\%20Slides\%20Dec\%202010. pdf.

27. Subfertiliteit. 1998, Nederlandse Vereniging voor Obstetrie en Gynaecologie. Available from: http://www.nvog.nl/.

28. Fertility: assessment and treatment for people with fertility problems update. 2013, National Institute for Health and Clinical Excellence (NICE). Available from: http://guidance.nice.org.uk/CG156.

29. Haggerty, CL, Gottlieb, SL, Taylor, BD, et al. Risk of sequelae after Chlamydia trachomatis genital infection in women. The Journal of infectious diseases, 2010. 201 Suppl 2: p. S134-155.

30. van Bergen, J, Gotz, H, Richardus, JH, et al. Prevalence of urogenital Chlamydia trachomatis infections in the Netherlands suggests selective screening approaches. Results from the PILOT CT Population Study. Drugs of today, 2006. 42 Suppl A: p. 25-33.

31. van Bergen, JE, Fennema, JS, van den Broek, IV, et al. Rationale, design, and results of the first screening round of a comprehensive, register-based, Chlamydia screening implementation programme in the Netherlands. BMC infectious diseases, 2010. 10: p. 293.

32. van den Broek, IV, van Bergen, JE, Brouwers, EE, et al. Effectiveness of yearly, register based screening for chlamydia in the Netherlands: controlled trial with randomised stepped wedge implementation. BMJ, 2012. 345: p. e4316.

33. RIVM. Chlamydia: Hoe vaak komt het voor en hoeveel mensensterven eraan? 2012; Available from: http://www.nationaalkompas.nl/gezondheid -en-ziekte/ziekten-en-aandoeningen/infectieziekten-en-parasitaireziekten/soa/chlamydia/omvang/.

34. Koedijk, FD, van Bergen, JE, Dukers-Muijrers, NH, et al. The value of testing multiple anatomic sites for gonorrhoea and chlamydia in sexually transmitted infection centres in the Netherlands, 2006-2010. International Journal of STD \& AIDS, 2012. 23(9): p. 626-631.

35. Beurskens, MP, Maas, JW and Evers, JL. [Subfertility in South Limburg: calculation of incidence and appeal for specialist care]. Nederlands tijdschrift voor geneeskunde, 1995. 139(5): p. 235-238.

36. van Balen, F, Verdurmen, $J$ and Ketting, E. Choices and motivations of infertile couples. Patient education and counseling, 1997. 31(1): p. 1927.

37. Balen, AH. Infertility in Practice. Fourth edition ed. Reproductive Medicine and Assisted Reproductive Techniques Series 2014: CRC Press. 500. 
38. de Graaff, AA, Land, JA, Kessels, AG, et al. Demographic age shift toward later conception results in an increased age in the subfertile population and an increased demand for medical care. Fertility and sterility, 2011. 95(1): p. 61-63.

39. Geboorteregeling 2008. Centraal Bureau voor de Statistiek. Available from: www.cbs.nl.

40. Leeftijd moeder bij eerste kind per gemeente. 2010, Nationale Atlas Volksgezondheid Available from: http://www.zorgatlas.nl/ beinvloedende-factoren/demografie/geboorte-en-sterfte/leeftijdmoeder-bij-geboorte-eerste-kind-per-gemeente/.

41. Geboorte; leeftijd moeder. Centraal Bureau voor de Statistiek. Available from: http://statline.cbs.nl/StatWeb/ publication/?VW=T\&DM=SLNL\&PA $=37520 \& L A=N L$.

42. Gezondheid en zorg in cijfers 2012. Centraal Bureau voor de Statistiek. Available from: http://www.cbs.nl/NR/rdonlyres/B438B6AC-BAB54F71-A7F3-26EB01FE66EF/O/2012c156puberr.pdf.

43. ART Factsheet. European Society for Human Reproduction and Embryology (ESHRE). Available from: http://www.eshre.eu/Guidelinesand-Legal/ART-fact-sheet.aspx.

44. den Hartog, JE, Lardenoije, CM, Severens, JL, et al. Screening strategies for tubal factor subfertility. Human reproduction, 2008. 23(8): p. 18401848.

45. Broeze, KA, Opmeer, BC, Van Geloven, N, et al. Are patient characteristics associated with the accuracy of hysterosalpingography in diagnosing tubal pathology? An individual patient data meta-analysis. Human reproduction update, 2011. 17(3): p. 293-300.

46. Swart, P, Mol, BW, van der Veen, F, et al. The accuracy of hysterosalpingography in the diagnosis of tubal pathology: a metaanalysis. Fertility and sterility, 1995. 64(3): p. 486-491.

47. Bonneau, C, Chanelles, O, Sifer, C, et al. Use of laparoscopy in unexplained infertility. European journal of obstetrics, gynecology, and reproductive biology, 2012. 163(1): p. 57-61.

48. Land, JA, Evers, JL and Goossens, VJ. How to use Chlamydia antibody testing in subfertility patients. Human reproduction, 1998. 13(4): p. 1094-1098.

49. Manolio, TA, Chisholm, RL, Ozenberger, B, et al. Implementing genomic medicine in the clinic: the future is here. Genetics in medicine : official journal of the American College of Medical Genetics, 2013. 15(4): p. 258-267. 



\section{CHAPTER 5}

The attitudes of the Dutch gynaecologists towards the addition of genetic testing in the diagnosis of tubal factor infertility (TFI)

J Malogajski, M Jansen, S Ouburg, E Ambrosino, CB Terwee, SA Morré

Publication in preparation 


\section{Abstract}

The ability of genomics to have an impact on clinical practice depends, among other factors, on clinicians' knowledge and attitudes regarding genetic tests. Research of the host genetic factors influencing the course and outcome of Chlamydia trachomatis infections has the potential to improve the accuracy of diagnosing tubal factor infertility (TFI) through genetic testing. Genetic variations, such as single nucleotide polymorphisms (SNPs) in pathogen recognition receptors (PRR) genes are involved in the recognition of Chlamydia can influence the course and outcome of the infection. Identifying and combining multiple SNPs in PRR genes and their linked pathways, and correlating them with infection outcomes offers an opportunity for the development of a new TFI screening strategy. In order to explore Dutch gynaecologists' attitudes towards the introduction of genetic testing in the routine screening for TFI we preformed research in two phases. In the first phase we performed interviews with experienced reproductive specialists working in 4 Dutch Academic Hospitals. We used results of these interviews to develop a questionnaire and surveyed 48 doctors working at Ob/Gyn departments of the Academic hospitals in Groningen, Maastricht and Utrecht. Our aim was to understand what respondents perceive as barriers and facilitating factors for the use of genetic information for TFI screening. Majority of participants agreed that addition of genetic markers to the CAT, in screening for tubal pathology could change the sensitivity of testing and more than half expressed the opinion that their patients have preferences for non-invasive testing. Results showed that all the respondents, regardless of their position at the department or the hospital they work in indicated cost-effectiveness as an important factor in gaining their support for the new screening strategy. Clinical utility is recognized as the most import indicator of the quality of a genetic test. 


\section{Introduction}

Advances in genomics are contributing to the growing understanding of the role genes have as underlying causes in increasing number of diseases. The importance of genetic information in appropriate clinical management of these diseases is increasing [1, 2].Using genetic information in routine clinical practice in order to provide high quality healthcare is one of the major hallmarks of modern medicine.

Advances in genetics have had clinical implications for gynecology practice. Indeed, genetic testing found its first application in gynecology/obstetrics more than three decades ago with prenatal genetic screening for common birth defects during pregnancy [3]. More recently, preimplantation genetic screening (PGD) has been introduced in the field of assisted reproductive medicine, aiming to improve pregnancy rates in older women undergoing in vitro fertilization (IVF) [4]. In the meantime, using genetic testing for assessment of patient's risk for hereditary breast and ovarian cancer became a routine in obstetric and gynecologic practice [5].

At the same time, there have been new developments in the research of host genetic determinants of infectious diseases. Indeed, recent developments in immunogenetic research of Chlamydia trachomatis urogenital infections, one of the most common causes of damage to the Fallopian tubes and consequent tubal factor infertility (TFI) has the potential to create a link between the fields of gynecology and infectious diseases and improve the accuracy of diagnosing tubal factor infertility (TFI) through genetic testing.

One in six couples worldwide are suffering from infertility [6]. In up to $30 \%$ of all cases infertility is attributed to tubal factors [7]. Chlamydia trachomatis lower genital infection is an important risk factor for the development of tubal pathology and consequent tubal factor infertility (TFI). As much as $45 \%$ of tubal pathology can be linked to a previous Chlamydia infection [8].

Despite the fact that chlamydial infections are in most cases asymptomatic and therefore not treated with antibiotics, almost half of the women with the asymptomatic infection clear it without any within a year [9]. However, other women are prone to persistent infections and consequent complications such as PID, ectopic pregnancy TFI [10]. Up to $40 \%$ of differences in the clinical course 
of Chlamydia infections among individuals can be attributed to host genetic factors [ 11 1]. Genetic variations, such as single nucleotide polymorphisms (SNPs) in PRR genes involved in the recognition of Chlamydia and in the initiation of an adequate immune response can contribute to higher or lower risk of Chlamydia induced TFI [12, 13]. Identifying and combining multiple SNPs in PRR genes and their linked pathways, and correlating them with infection outcomes, offers an opportunity for the development of a new TFI screening strategy which would be more accurate in identifying patients at low or high risk of having tubal pathology.

At the moment, Chlamydia antibody test (CAT) and Hysterosalpingography (HSG) are the most commonly used screening tests for TFI in the routine fertility work up in the Netherlands. Sensitivity and specificity of the most accurate CATs are assessed at approximately 60\% and 85-90\% respectively [14], for HSG these numbers are $53 \%$ and $87 \%[15,16]$. This means that some women who test negative in these screening tests have tubal pathology, and a number of women testing positive do not. Therefore, there is space for increase in accuracy of TFI screening. Combining CAT testing with genetic test early in the fertility work up could increase the accuracy of screening since CAT and genetic test combined would have higher PPV and NPV than any of the existing screening strategies.

Ultimately though, the ability of genomics to have an impact on clinical practice depends on clinicians' knowledge and attitudes regarding genetic tests. Clinicians make decisions about ordering genetic tests and they are also responsible for interpreting and explaining the results to the patients [17]. Measuring their' experiences and attitudes towards the use of genetic tests is increasingly recognized as an important tool for understanding barriers and facilitating factors for the use of genetic information in diagnosis and treatment of diseases. [18-21].

The objective of our study is to explore Dutch gynaecologists' attitudes towards the introduction of genetic testing in the routine screening for TFI and to evaluate their knowledge about the genetic background behind different courses and outcomes of Chlamydia infections. 


\section{Materials and Methods}

In performing our study we used both qualitative and quantitative methods (mixed method). We performed our study in two phases, in the first phase we performed interviews with experienced reproductive specialists working in 4 Dutch Academic Hospitals. We used results of these interviews to develop a questionnaire for the doctors working at Ob/Gyn departments about their attitudes towards the addition of genetic testing in the diagnosis of TFI. In the second phase, using the questionnaire, we surveyed 48 doctors working at $\mathrm{Ob} /$ GYN departments and providing fertility care in 3 Dutch Academic hospitals in order to investigate what they perceive to be major challenges and major facilitating factors in introducing genetic testing in routine fertility work up.

\section{Interviews}

Four semi structured interviews with gynaecologists working in Dutch academic hospitals, were conducted in February and March of 2013. Before conducting them we created some guidelines and decided on the main issues that would be discussed, however we did not insist on always following the same sequence of the questions or on the exact formulation used in the guidelines. The purpose of these interviews was to explore the topic of infertility in general, to get information and an inner perspective on current guidelines and practices in the screening for TFI. The interviews also provided information on potential facilitators and barriers for broad implementation of genetic testing in the diagnosis of TFI. The interviews served as a foundation for building questionnaires. Three interviews were conducted face-to-face and one via Skype. All the interviews were recorded and later transcribed. The transcripts of interviews were later analyzed.

Development of the questionnaires Based on the analysis of interviews we made a theoretical framework in order to group and give an overview of the factors which emerged as important in influencing the attitudes towards introduction of genetic testing in TFI investigation. 
Table 1. Overview of the factors influencing the attitudes toward the introduction of genetic testing into diagnosis of TFI

\begin{tabular}{|l|l|l|l|}
\hline FACTOR & ELEMENTS & ASPECTS \\
\hline $\begin{array}{l}\text { Added value for } \\
\text { clinical investigation }\end{array}$ & $\begin{array}{l}\text { Increasing diagnostic } \\
\text { accuracy }\end{array}$ & Sensitivity, Specificity, NPV, PPV \\
\hline & $\begin{array}{l}\text { Lower number of } \\
\text { misdiagnosis }\end{array}$ & \\
\hline & & Costs & Effects \\
\hline Quality of the test & Cost-effectiveness & Identifying patient's clinical status correctly \\
\hline & Clinical validity & Risks and benefits resulting from test use \\
\hline & Clinical utility & \multicolumn{2}{|l|}{} \\
\hline & & Fear from a procedure \\
\hline Patient preferences & Invasiveness & Fear from complications \\
\hline & $\begin{array}{l}\text { Complications related to a } \\
\text { diagnostic procedure }\end{array}$ & & Competition between different competences \\
\hline Roles/competences & $\begin{array}{l}\text { Collaborating with clinical } \\
\text { geneticists }\end{array}$ & $\begin{array}{l}\text { Optimizing timing } \\
\text { in the diagnostic } \\
\text { investigation }\end{array}$ & $\begin{array}{l}\text { Optimizing timing of } \\
\text { beginning a treatment }\end{array}$ \\
\hline Time & Saving time & \multicolumn{2}{|l}{} \\
\hline
\end{tabular}

Five factors were most frequently mentioned in the interviews and were identified as relevant for the future questionnaire (Table 1). The added value, that genetic testing would bring to the routine fertility work-up, the cost of the genetic testing and additional costs that would mean for the healthcare system, patient's preferences for non-invasive testing and their fear of complications associated with certain diagnostic procedures and the issue of gynecologist's roles and competences related to the introduction of genetic testing in routine fertility work up were used as the foundation for the questionnaire statements. In order to assure content validity of the questionnaire we sent the first draft of the questionnaire to an expert panel consisted of a gynecologist with more than 30 years of experience in fertility care and an epidemiologist with background expertise in the development and evaluation of outcome measures. Based on their feedback we made changes in the questionnaire. 
The final draft of the questionnaire consisted of 24 questions divided in 3 sections (see Appendix). In the first section we asked the respondents about their demographics and the level of experience in gynecology. In the second section participants were asked to answer question related to immunogenetic research of Chlamydia trachomatis and its possible implications for the clinical practice. Finally, in the third section we asked the respondents to express their attitudes towards introduction of genetic testing in screening for Chlamydia induced TFI. They expressed their attitudes, ranging from very negative to very positive, by showing how strongly they agreed or disagreed with our composed statements. We offered 6 response alternatives to each statement: agree, partly agree, neutral, partly disagree, disagree, and no opinion.

\section{Conducting the survey}

In the period from May to July 2014 we visited Academic hospitals in Maastricht, Groningen and Utrecht. These visits were organized in advance with the chiefs of the Gynecology departments of the hospitals. The participation in the survey was voluntary. The questionnaires were distributed to the gynaecologists attending the meetings and were filled in and returned on the spot.

\section{Statistical analysis}

The data from questionnaires are gathered in a Microsoft Access database, and analyzed with Microsoft Excel. The three parts of the questionnaire are each displayed separately. First the demographics are described, followed by the answers to the questions on the second part of the questionnaire. Each question and corresponding answers are shown in a bar diagram, comparing the relative outcomes of the responses. Lastly, the scores on the statements in part three of the questionnaire are provided, also in bar diagrams with the relative scores.

\section{Results}

Participants of the survey were 48 physicians working at the OB/GYN departments of the 3 Dutch Academic Hospitals, 19 at the Maastricht University Hospital (MUMC), 17 at the University Medical Center of Groningen (UMCG) and 12 at the University Medical Center Utrecht (UMCU). Main characteristics of the participants are summarized in Table 2,3. Most of the respondents, $29.2 \%$, were resident physicians at Gynecology departments followed by $25 \%$ 
of respondents who checked the box "Other" and mostly worked as IVF and fertility specialists at Gynecology departments. According to the age groups, most of the participants, 27 of them were between 25 and 35 years of age.

Table 2. Demographic characteristics of the participants

\begin{tabular}{|c|c|c|c|c|}
\hline \multirow[t]{3}{*}{ Sex } & & & $\mathrm{N}$ & $\%$ \\
\hline & Male & & 7 & 14.6 \\
\hline & Female & & 41 & 85.4 \\
\hline \multirow[t]{5}{*}{ Position } & Resident Physician & & 14 & 29.2 \\
\hline & Physician & & 6 & 12.5 \\
\hline & Gynaecologist & & 9 & 18.8 \\
\hline & Intern & & 7 & 14.6 \\
\hline & Other & & 12 & 25.0 \\
\hline Position & Female & $\%$ & Male & $\%$ \\
\hline Gynaecologist & 6 & 66.7 & 3 & 33.3 \\
\hline Intern & 6 & 85.7 & 1 & 14.3 \\
\hline Other & 11 & 91.7 & 1 & 8.3 \\
\hline Physician & 5 & 83.3 & 1 & 16.7 \\
\hline Resident physician & 13 & 92.9 & 1 & 7.1 \\
\hline
\end{tabular}

In the questionnaire we asked respondents about the opinions regarding possible implications of Chlamydia trachomatis immunogenetic research for their clinical practice. Answering the question whether genetic information could be used in the screening of Chlamydia induced subfertility more than $60 \%$ of participants gave positive answers (Figure 1). Giving their opinion about the ability of the combination of tests (CAT\& host genetic markers) to change the sensitivity of tubal factor subfertility diagnosis, $77 \%$ of the respondents believe that a combination of CAT and genetic markers will increase the sensitivity of subfertility diagnosis (Figure 2). 53 \% of respondents would be willing to support introduction of genetic test in the screening for tubal factor infertility (Figure 3). Regarding the location where the combination of tests CAT and genetic markers should be performed, $83 \%$ of respondents agreed that the testing should be performed at the gynecologist's office (Figure 4). 
In the questionnaire we also asked the participants to express their attitudes towards the introduction of genetic testing in screening for Chlamydia induced TFI. Each statement came with 6 response alternatives: agree, partly agree, neutral, partly disagree, and no opinion. In the first statement $63 \%$ of the respondents at least partially agrees that the adding genetic tests to CAT will increase the diagnostic accuracy (Figure 5). Expressing their attitudes about the ability of genetic testing to reduce the number of false positive and false negative results, $45 \%$ of the respondents at least partially agreed that the introduction of genetic tests in the diagnosis of subfertility will reduce their
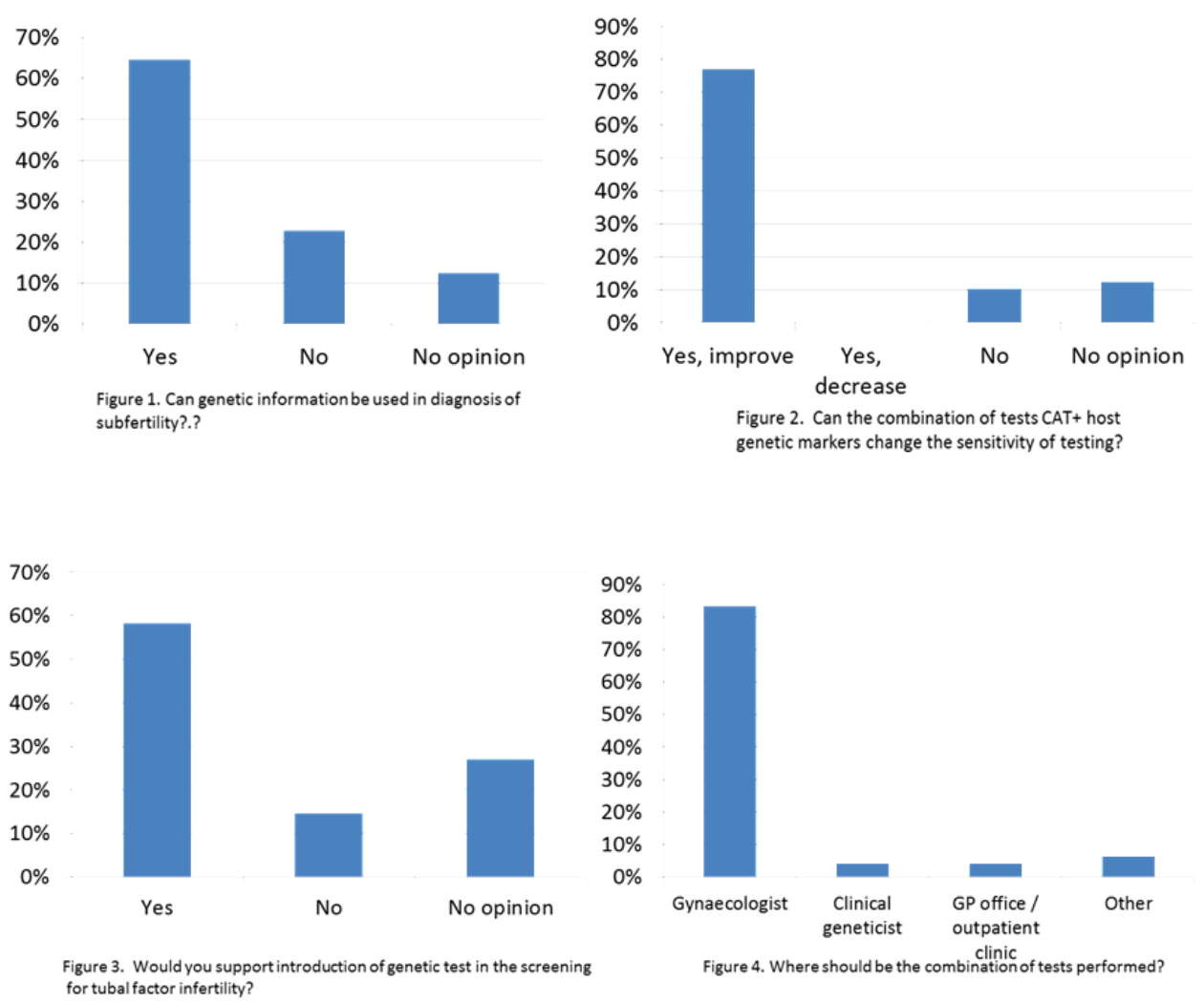

number (Figure 6). $31 \%$ of participants disagreed with the statement that the combination of genetic test and CAT would, in fact, prolong the time needed to arrive at the diagnosis, compared to $10 \%$ who expressed the attitude that the combination of tests will increase the time to diagnosis (Figure 7). Asked about the costs of genetic testing, $60 \%$ respondents agreed and partially agreed that genetic tests are too expensive (Figure 8). 
Asked about what factors related to the characteristics of the test would influence their support for the introduction genetic markers the most, respondents demonstrated following attitudes. Cost-effectiveness was very well supported, $100 \%$ of respondent found it important (Figure 9.), as were clinical validity and utility, by around half of respondents (Figures 9, 10, 11, 12).
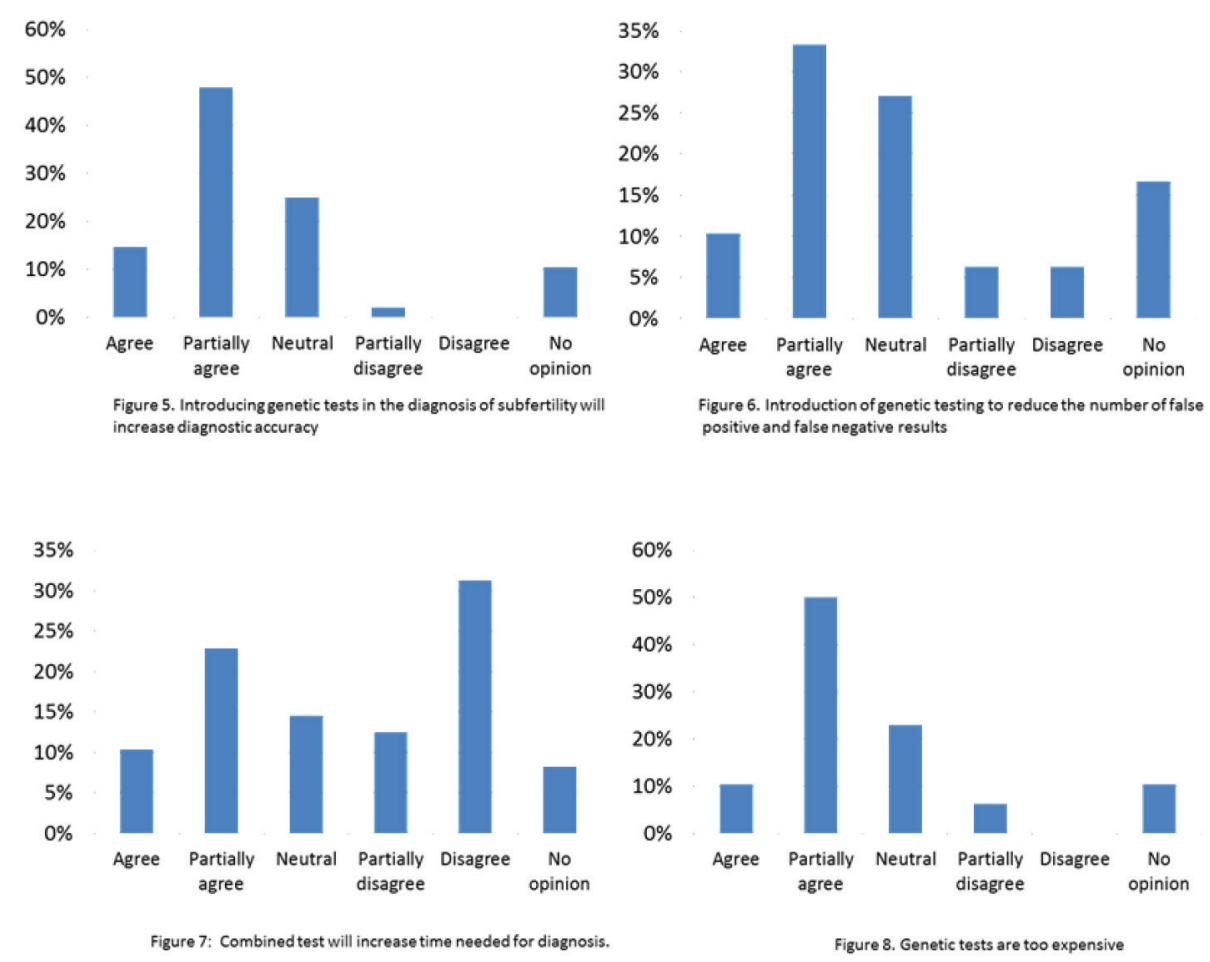

Expressing the attitudes about their patients' preferences when it comes to fertility investigation, 55\% of respondents stated that their patients prefer non-invasive testing (Figure 13.), more than half also agreed that their patients would prefer genetic testing over HSG and laparoscopy (Figures 14 and 15). Most respondents stated that they would consider their patients' preferences for the testing of tubal pathology (Figure 16.) 


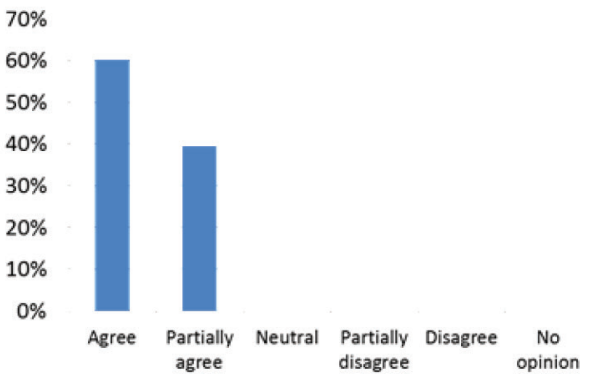

Figure 9 . I would support the introduction of the test if it was cost-effective

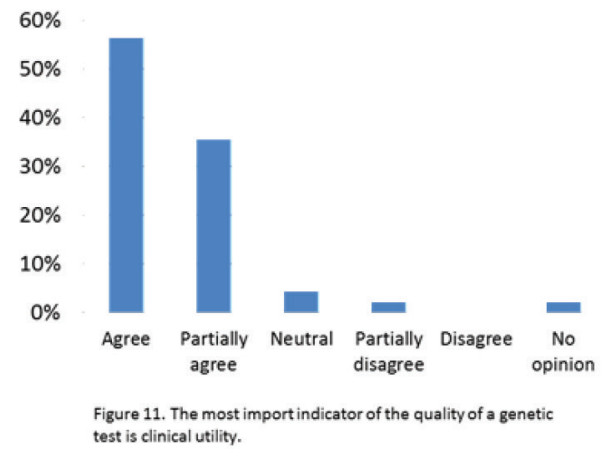

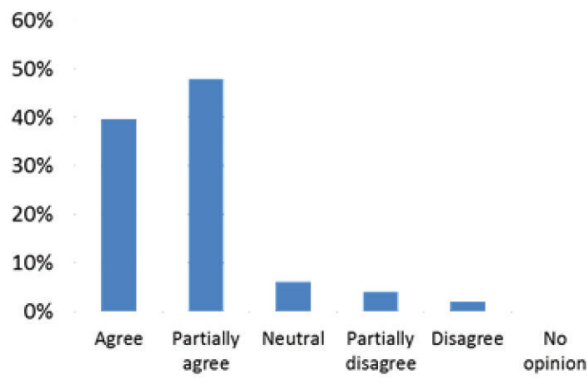

Figure 10. The most import indicator of the quality of a genetic test is clinical validity

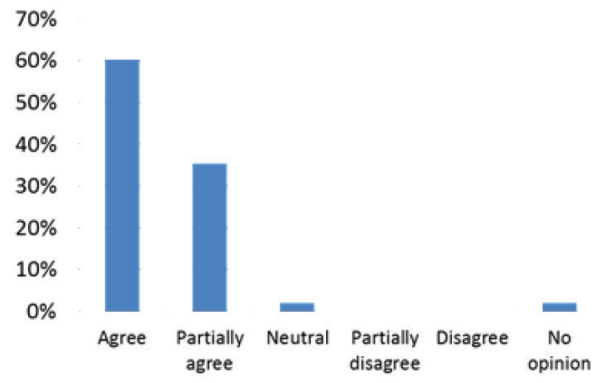

Figure 12. I would supportintroduction of a genetic test if the clinical validity and utility have been proven.

Responding to the statement about to the possible change that introduction of genetic test would bring to the role of gynaecologists in the diagnosis of tubal pathology, most of respondents did not feel that such a development would reduce their role in the diagnosis (Figure 17). However, majority did state that they would require additional training if a genetic test was added to the diagnosis of subfertility (Figure 18.)
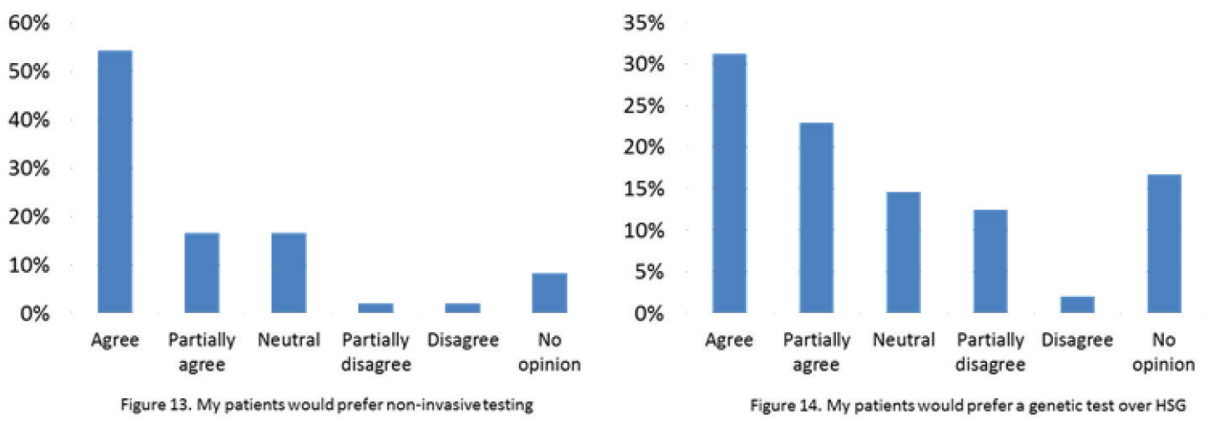

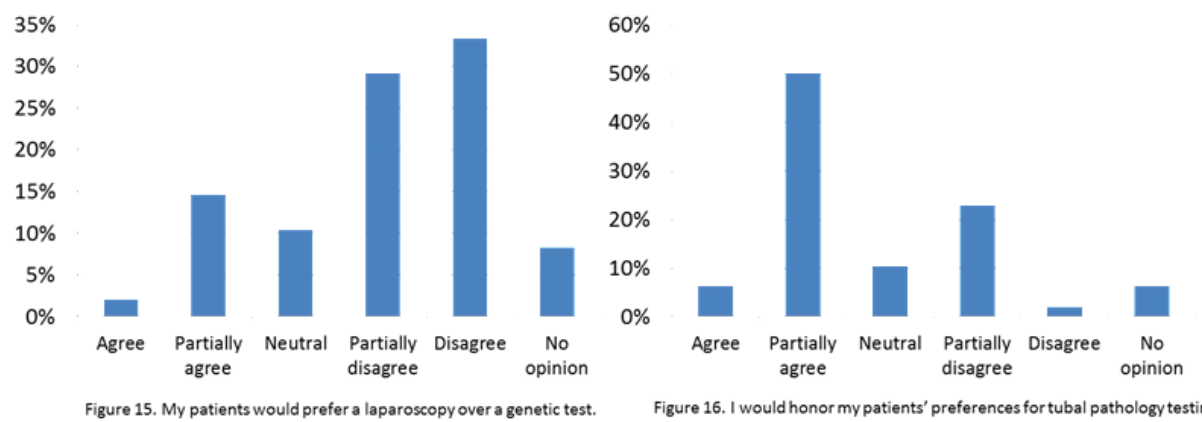

Figure 16. I would honor my patients' preferences for tubal pathology testing

\section{Discussion and Conclusion}

The present study was designed to investigate the Dutch gynaecologists' attitudes towards the introduction of genetic testing in the routine screening for TFI, and to identify factors they perceive as barriers and facilitators for the introduction of genetic testing in the current Dutch fertility care.
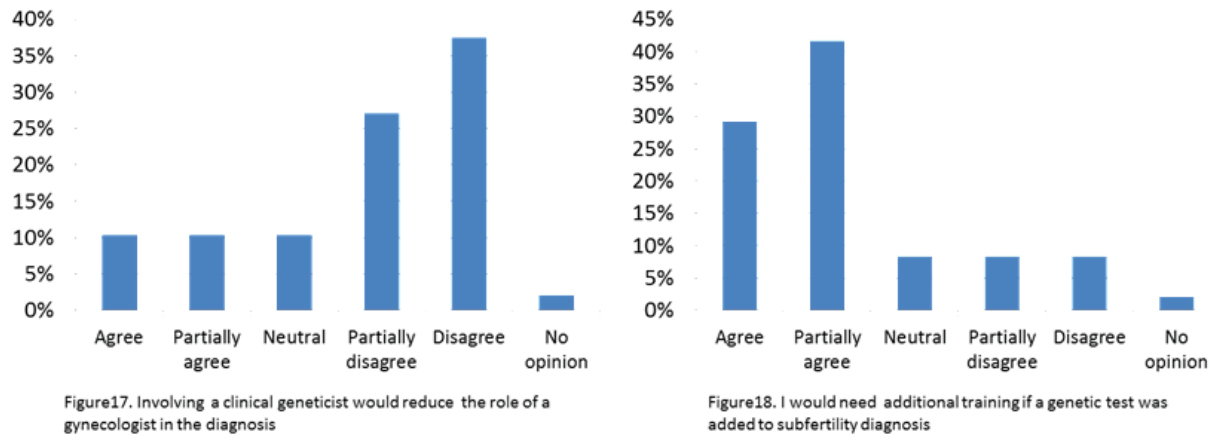

Several questions/statements have been identified as the major unifiers of participants' opinions and attitudes toward genetic testing. Cost-effectiveness was indicated by $100 \%$ of participants as an important factor in gaining their support for the new screening strategy regardless of their position at the department or the hospital they work in. Clinical utility is recognized as the most import indicator of the quality of a genetic test with $56 \%$ of the respondents fully agreeing that clinical utility is the most important indicator. Clinical utility is considered somewhat more important than clinical validity. These results are in concordance with a finding of an earlier study about physicians' attitudes toward genetic 
testing which found cost effectiveness and clinical utility to major concerns expressed. [22].

Another example of a uniting position among participants is their attitude about the expenses associated with genetic testing. Genetic tests are considered too expensive by the majority of the participants. We found no difference in opinions between participants in different academic hospitals. However, physicians were mostly neutral, $50 \%$.

When it comes to the potential of Chlamydial host genetic factors to improve the accuracy of the tubal pathology prediction and the support for the introduction of genetic tests in subfertility diagnosis gynecologist seem to be more "optimistic" than the other participants.

Asked if the addition of genetic markers to the CAT, in screening for tubal pathology can change the sensitivity of the diagnosis $77 \%$ of the respondents gave a positive answer, all the participating gynaecologists believed so. Also gynaecologists are the most positive about their support for the introduction of genetic tests in subfertility investigation. Out of $58 \%$ of respondents who supported the introduction of the test $89 \%$ were gynaecologists.

Interestingly enough, when asked if, in general, host genetic factors could be used in diagnosis of Chlamydia trachomatis induced subfertility, only $67 \%$ of gynaecologists expressed positive opinion. The highest support came from the interns ( $86 \%)$ followed by the physicians (83\%). Younger respondents seem more likely to support the use of host genetic factors.

We also got some contradicting results related to the many respondents (more than 60\%) believes that the diagnostic accuracy increases with genetic testing, but only $45 \%$ accepts that the number of false positive/negative results will be reduced by the genetic testing.

As the most polarizing questions we identified the question about the effect genetic test would have on the time needed for diagnosis. Around one third of the respondents agreed that combined test would increase time to diagnosis at the same time $31 \%$ disagreed with this notion. Most gynaecologists (56\%) believed that the time to diagnosis will be reduced, while $57 \%$ of the resident physicians and $42 \%$ of the 'others' (partially) agree that time to diagnosis will increase. 
Responding to the statement about the need for additional training if a genetic test was added to subfertility diagnostic procedures. Most respondents agreed they would need additional training. Gynaecologists and resident physicians felt the most that they would require additional training. Also, older respondents were more likely to reply that they would require additional training.

When it comes to the participants' attitudes toward their patients' preferences tubal pathology investigation, 55\% of them replied that their patient's prefer noninvasive testing. Only $6 \%$ of participants agreed to honor their patients' preferences. Most respondents (around 50\%) would, however, consider the preferences of their patients.

In conclusion, in spite of the high percentage of respondents who believe that addition of genetic markers to CAT, in routine screening for TFI can change the sensitivity of the diagnosis, respondent perceive some barriers for the implantation of such a test. All the respondents, regardless of their position at the department or the hospital they work in agreed that cost-effectiveness is an important factor in gaining their support for the new screening strategy. Clinical utility is recognized as the most import indicator of the quality of a genetic test. In addition to the justified expectations of the proven cost-effectiveness and clinical utility of the new screening strategy, perceived high costs associated with genetic testing pose the biggest barrier in acceptance of genetic testing in diagnosis of TFI. 


\section{REFERENCES}

1. Collins, FS and McKusick, VA. Implications of the human genome project for medical science. The Journal of American Medical Association , 2001. 285(5): p. 540-544.

2. Guttmacher, AE, Porteous, ME and McInerney, JD. Educating health-care professionals about genetics and genomics. Nature Reviews Genetics, 2007. 8(2): p. 151-157.

3. Lippman, A. Prenatal genetic testing and screening: constructing needs and reinforcing inequities. The American Journal of Medicine, 1991. 17 : p. 15.

4. McGowan, ML, Burant, CJ, Moran, R, et al. Patient education and informed consent for preimplantation genetic diagnosis: health literacy for genetics and assisted reproductive technology. Genetic Medicine, 2009. 11 (9): p. 640-645.

5. Force, USPST. Genetic Risk Assessment and BRCA Mutation Testing for Breast and Ovarian Cancer Susceptibility: Recommendation Statement: United States Preventive Services Task Force. The Internet Journal of Oncology, 2004. 3(1).

6. ESHRE. Assisted Reproductive Technology (ART) Factsheet. Available from: http://www.eshre.eu/Guidelines-and-Legal/ART-fact-sheet.aspx.

7. Evers, JL. Female subfertility. Lancet, 2002. 360(9327): p. 151-159.

8. Price, MJ, Ades, AE, Welton, NJ, et al. How much tubal factor infertility is caused by Chlamydia? Estimates based on serological evidence corrected for sensitivity and specificity. Sexually transmitted diseases, 2012. 39(8): p. 608-613.

9. Morré, SA, van den Brule, AJ, Rozendaal, L, et al. The natural course of asymptomatic Chlamydia trachomatis infections: $45 \%$ clearance and no development of clinical PID after one-year follow-up. International Journal of STD \& AIDS, 2002. 13 Suppl 2: p. 12-18.

10. Paavonen, $\mathrm{J}$ and Eggert-Kruse, W. Chlamydia trachomatis: impact on human reproduction. Human reproduction update, 1999. 5(5): p. 433447.

11. Bailey, RL, Natividad-Sancho, A, Fowler, A, et al. Host genetic contribution to the cellular immune response to Chlamydia trachomatis: Heritability estimate from a Gambian twin study. Drugs of today, 2009. 45 Suppl B: p. 45-50.

12. den Hartog, JE, Ouburg, S, Land, JA, et al. Do host genetic traits in the bacterial sensing system play a role in the development of Chlamydia trachomatis-associated tubal pathology in subfertile women? BMC infectious diseases, 2006. 6: p. 122. 
13. Morré, SA, Karimi, $\mathrm{O}$ and Ouburg, S. Chlamydia trachomatis: identification of susceptibility markers for ocular and sexually transmitted infection by immunogenetics. FEMS immunology and medical microbiology, 2009. 55(2): p. 140-153.

14. Land, JA, Van Bergen, JE, Morré, SA, et al. Epidemiology of Chlamydia trachomatis infection in women and the cost-effectiveness of screening. Human reproduction update, 2010. 16(2): p. 189-204.

15. Broeze, KA, Opmeer, BC, Van Geloven, N, et al. Are patient characteristics associated with the accuracy of hysterosalpingography in diagnosing tubal pathology? An individual patient data meta-analysis. Human reproduction update, 2011 . 17(3): p. 293-300.

16. Swart, P, Mol, BW, van der Veen, F, et al. The accuracy of hysterosalpingography in the diagnosis of tubal pathology: a metaanalysis. Fertility and sterility, 1995. 64(3): p. 486-491.

17. Baars, MJ, Henneman, L and Ten Kate, LP. Deficiency of knowledge of genetics and genetic tests among general practitioners, gynaecologists, and pediatricians: a global problem. Genetic Medicine, 2005. 7(9): p. 605-610.

18. Klabunde, CN, Willis, GB and Casalino, LP. Facilitators and barriers to survey participation by physicians: a call to action for researchers. Evaluation and the Health Professions, 2013. 36(3): p. 279-295.

19. Klitzman, R, Chung, W, Marder, K, et al. Attitudes and Practices Among Internists Concerning Genetic Testing. Journal of Genetic Counseling, 2013. 22(1): p. 90-100.

20. Salm, M, Abbate, K, Appelbaum, P, et al. Use of genetic tests among neurologists and psychiatrists: knowledge, attitudes, behaviors, and needs for training. Journal of Genetics Counseling, 2014. 23(2): p. 156163.

21. Teng, I and Spigelman, A. Attitudes and knowledge of medical practitioners to hereditary cancer clinics and cancer genetic testing.

Familial Cancer, 2014. 13(2): p. 311-324.

21. Freedman, AN, Wideroff, L, Olson, L, et al. US physicians' attitudes toward genetic testing for cancer susceptibility. American journal of medical genetics. Part A, 2003. 120A(1): p. 63-71. 
CHAPTER 6

GENERAL DISCUSSION 
The topic of this thesis is the translation of genomic information into clinical practice for infectious diseases, and specifically the potential of host genomic determinants to improve the tubal factor infertility (TFI) work up in the Netherlands. The first part of the thesis focuses on the translation of genomic information and on the development of genomic applications for everyday clinical practice of sexually transmitted infectious of high public health relevance. In the first part of the thesis we also explore the role of biobanks as an important element of research infrastructure in the process of integration of genomebased knowledge in clinical practice of infectious diseases. The second part of the thesis focuses on the potential of Chlamydia trachomatis host immunogenetic research to improve accuracy of TFI screening through introduction of genetic biomarkers into routine fertility work up. The current chapter will discuss the findings presented in this thesis and the implications of these findings for future research.

\section{Public Health Genomics of Infectious diseases}

One of the aims of Public Health Genomics is to identify opportunities for genomics to improve health and to demonstrate how genomic information has potential to transform healthcare from promise to reality [1]. In the first part of the thesis we focused on exploring that potential in the field of three infectious diseases of high public health relevance.

As described in Chapter 1 , in our review of the translational potential of basic genomic and genetic findings for Human Immunodeficiency Virus (HIV), Chlamydia trachomatis (CT), and Human papillomavirus (HPV), we looked for promising examples of translation of basic scientific discovery into a clinical application. In concordance with a prevailing sense of lack of integration of the new technologies into clinical practice [2], in the review we found scarce examples of the current application of genomic/genetic information in the aforementioned field of infection diseases. 
HIV

HIV remains one of the biggest public health challenges. The incidence of HIV is decreasing, but due to anti-retroviral therapy (ART), the prevalence continues to increase and 35 million people were living with the infection at the end of 2013 [3]. Host genetic variation related with HIV/AIDS has been a topic of interest for researchers last 30 years [4].

Host genetic studies aim to increase understanding of inter-individual differences in, susceptibility to and outcome of infectious diseases. In the field of HIV research, high numbers of host genomic determinants were found to influence HIV infection, progression and outcome. However, few of these associations were positively confirmed. Chemokine receptor and chemokine ligand genes as well as HLA class I and related genes are identified as the most influential in modulating the susceptibility and outcome of HIV infection [5-10]. In the case of HIV infection, the research of host genetic determinants has had so far predominantly pharmacogenomics applications.

Absence or reduction of the number of CCR5 co-receptors on the CD4+ cell surface has been identified as HIV resistance factor and served as a foundation for the development of a family of drugs known as entry inhibitors. At the time of this study, only two entry inhibitors were approved for the treatment of HIVinfected patients. Maraviroc is a CCR5 antagonist and Enfuvirtide, is a fusion inhibitor, it disables conformational changes in gp41 that drive membrane fusion [ 11,12$]$. The number of approved entry inhibitors has not changed since. The development of Vicriviroc, also a CCR5 co-receptor antagonist designed to block the virus from penetrating the cells, which was considered promising at the time of the study has since been cancelled at the end of 2010 . The research on other promising molecules continues [13].

Research on HLA class I genes lead to important pharmacogenomics application. Patients expressing $B * 5701$ allele are hypersensitive to an antiretroviral drug called Abacavir prescribed to patients with HIV. By routinely testing every potential drug recipient and identifying patients with the mentioned allele, very serious, and possibly life threatening drug reactions are avoided [14].

The focus of more recent research in HIV host genomics is on the analysis of killer cell immunoglobulin-like receptor (KIR) genes and HLA class II alleles 
[15] has been established recently that HIV + individuals expressing the HLA class II allele DR B $1{ }^{*} 1303$ show significantly lower viral loads. The protective effect of this allele is found to be independent of sex, ethnical background or HIV virus subtypes [16]. Additionally, Alter et al. suggested that NK cells have an important role in the control of HIV infection. They demonstrated that KIR positive NK cells can put immunological pressure on HIV-1. In order to evade the immune response, the virus is evolving through so called escape mutations, therefore NK cells might have a very important role in contributing to viral evolution [17]. The evolution of HIV at the population level is driven by these alternative escape mechanisms and understanding all the factors behind them is of highest importance for designing successful HIV vaccines. [18]. The review of the recent HIV research demonstrates renewed efforts for the HIV vaccine development [19].

Recent insights into HIV host genomic research indicate change in research approaches in the near future. HIV host genetic research is moving away from candidate gene studies and genome wide association studies (GWAS), responsible for so far identified host genetic factors, towards investigating small-scale changes such as single-nucleotide variants through comprehensive sequencing [20].

\section{HPV}

For the development of cervical cancer, infection with an oncogenic type of human papillomavirus (HPV) is crucial but not sufficient. Variability of factors that control the immune response to infection has a great impact on the outcome of the infection [21].

Review of the host genetic variants influencing HPV infection identified several alleles associated with higher risk of persistent HPV infection and development of cervical cancer. There were also polymorphisms identified to have a protective role, making an individual less susceptible to cervical cancer [22, 23]. So far these findings have not been used in the prevention or treatment of HPV infection. Indeed, we found (Chapter 1) that when it comes to using genetic information in prevention of cervical cancer, methylation based triage of highrisk human papilloma virus (hrHPV) offered the most promising possibilities. A number of tumor suppressor genes were found to have methylated gene 
promoters in cervical cancer tissue. Methylation of the promoters CCNA 1 and C130RF 18 in cervical scrapings was found to be strongly associated with CIN2 (moderate cervical intraepithelial dysplasia) and higher grade stages of cervical dysplasia [24] making them ideal for triage of patients positive for hrHPV. This combination of tests was considered a solution for a more effective stratification in the group of hrHPV positive women, by identifying those at high risk for cervical cancer and, at the same time, reducing the number of unnecessary referrals to colposcopy in those with negative methylation test.

Ongoing research on this topic confirms the importance of DNA methylation analysis of promoter regions of tumor suppressor genes as an objective triage tool for hrHPV positive women [25]. Recent research also suggest HPV multiplex ligation-dependent probe amplification assay (HPV MLPA) as a recommended triage method following hrHPV. The added value of recently developed HPV MLPA assay is in classifying samples as high risk based on the detection of a high viral load and/or viral integration in cytological samples. hrHPV screening has a high negative predictive value ( NPV ) of $97 \%$, but it's positive predictive value (PPV) is evaluated at 50\%, Combining hrHPV screening with HPV MLPA test and assessing additional risk factors such as viral load and the viral integration, has the potential of increasing the PPV of screening and reducing the number of unnecessary colonoscopies [26].

\section{Chlamydia trachomatis}

Chlamydia trachomatis is the cause of the most common sexually transmitted infection worldwide and it has a persisting high incidence of 90-100 million cases per year. Host genetic twin studies of Chlamydia have established that 40 $\%$ of the course and the outcome of the infection can be explained by host genetic factors [27]. The ultimate question is which factors are the most predictive, area of research which got much attention in the last 5 years. Chlamydia infection can be symptomatic or asymptomatic, the latter is more common, therefore most of the time this infection is not treated by antibiotics. Both symptomatic and asymptomatic infections can either spontaneously clear or ascend to the upper genital tract causing complications, such as salpingitis, pelvic inflammatory disease (PID), ectopic pregnancies and tubal factor infertility (TIF). The innate immune response relies on the proper recognition of bacterial peptidoglycan by Pathogen Recognition Receptors (PRRs) [28]. It has been established that 
these differences in the course and outcome of the infection are often determined by genetic variations, such as single nucleotide polymorphisms (SNPs), in genes encoding for Pathogen Recognition Receptors (PRRs) located on macrophages, vaginal and tubal epithelial cells or intracellularly. Carrying polymorphism in genes encoding PRRs, such as Toll Like Receptors (TLRs) or he Nucleotide-binding Oligomerization Domain Receptors (NODs), can put an individual at high risk for development of tubal pathology, or act in the protective way, protecting an individual from persistent Chlamydia infection and consequent complications. In Chapters 1 and 3 we provided an overview of host genetic factors related to Chlamydia infection. TLRs 2, 4 and 9 are well-researched TLR family members. Several studies observed that carrying SNPs in TLR4 has a role in making women more prone to infertility as a late complication of Chlamydia infection. Nevertheless, the exact role of TLR 4 in infertility has not been yet been fully understood [29, 30]. The likelihood of carrying TLR4 +896 A allele was found to be $2 x$ higher in women positive for Chlamydia trachomatis IgG antibodies ( CAT +) then in women without tubal pathology [30]. In a study aiming at understanding the role of two TLR2 SNPs in the susceptibility to infection and contribution to the development of tubal pathology, a statistically significant association between certain TLR2 haplotypes and the protection from tubal pathology [31]. Carrying two or more SNPs in TLR9, TLR4, CD14, and CARD15/NOD2 increased the risk of developing tubal pathology following Chlamydia infection [32]. Recent research confirmed the role of NOD 1 polymorphisms in the development of tubal pathology. Chlamydia IgG CAT (+) women with TFI were significantly more frequent carriers of a certain insertion polymorphism in the NOD 1 gene compared to CAT (-) women [33].

Immunogenetic research demonstrates that genetic and genomic markers influencing the course and outcome of Chlamydia infection could have successful application in the prediction of risk of Chlamydia trachomatis infection and of its late complications. Identifying genomic markers responsible for the course and outcome of Chlamydia infection and developing a screening test based on them would allow the discrimination between infertile women with high risk of tubal pathology versus infertile women with low risk of tubal pathology.

Recently, in a key study in the field of Chlamydia immunogenetic research, GWAS was used for the first time to scan for pathway-wide genomic differences between cases of scarring trachoma and controls [34, 35] 


\section{Relevance of biobanks}

As described in Chapter 2, biobanks have an increasing role in the field of genetic susceptibilities of infectious diseases. Large scale genome-wide association studies and candidate gene studies, performed on samples stored in biobanks are becoming essential for confirming new gene-infectious disease associations. Examining various networks and biobanks that specialize in infectious diseases we noticed a trend of increasing number of studies deriving from them. Our search confirmed another noticeable trend in infectious disease genomics, the development of nation-wide or transnational collections and building large consortia and biobanks networks [36]. A good example of this is the EpiGenChlamydia Consortium, European funded transnational research network which has been establishing biobanks and data warehouses aiming to perform comparative genomic and genetic epidemiology studies. The goal is to understand inter-patient differences in the course and outcome of Chlamydia infection and ultimately develop an accurate diagnostic tool for predicting the risk of tubal pathology [37].

For biobanks to realize their full potential in supporting the research on genetic susceptibilities of infectious diseases (and other fields of research), researchers should be able to freely access a number of them worldwide. Legal and ethical issues surrounding the establishment of global biobanking networks, which have been hindering this process for years, ought to be resolved for the benefit of population health. 


\section{Chlamydia induced infertility- genomic testing in the routine investigation of TFI}

Estimating the risk of tubal pathology following the infection is one of the biggest challenges of Chlamydia research [38] Almost half of all asymptomatic infected women will spontaneously clear the infection in one year [39]. The reported risk of infertility following Chlamydia infection shows great variation, from $2-4.5 \%$ to $30 \%$ women with a previous untreated Chlamydia infection are developing PID and $10-20 \%$ of them are developing TFI $[38,40]$.

As we described in Chapter 4, detecting C. trachomatis IgG antibodies is the most commonly used screening test for TFI in the Netherlands. In the group of subfertile women with TFI the incidence of CAT positivity is much higher compared to women without TFI. There are a number of studies reporting on the accuracy of CAT. Overall the sensitivity for tubal pathology of the most accurate CATs is assessed at approximately $60 \%$, with a specificity of $85-90 \%$ [41]. As we found out investigating strategies currently in place, in fertility care in the Netherlands there is no consensus on the sequence of diagnostic procedures in routine fertility work-up. CAT is performed alone or in combination with HSG, laparoscopy is used as a reference diagnostic standard (Chapter 4).

The main problem with TFI screening based on CAT, is the high numbers of false positive cases, in other words, high number of women with positive IgG Chalmydia trachomatis antibodies without tubal pathology are undergoing unneeded laparoscopies.

We proposed a screening strategy that would introduce SNPs-based analysis into routine TFI screening by combining CAT with genomic diagnostics early in the fertility work-up.

This combination of tests (CAT and genomic markers) would potentially have higher (PPV) and (NPV). Meaning that the number of women with positive CAT, who are at the moment considered at risk of tubal pathology would not undergo invasive diagnostic procedures if their genetic markers would not attribute them with the high risk of TFI (Figure 1). Further, despite being CAT negative and not considered at risk of TFI, a certain number of women, in fact, have tubal pathology. In their case, testing positive for high risk of TFI would speed up further investigations. Therefore, the overall outcome would be fewer women 
undergoing not needed diagnostic procedures and more women getting the intervention they need.

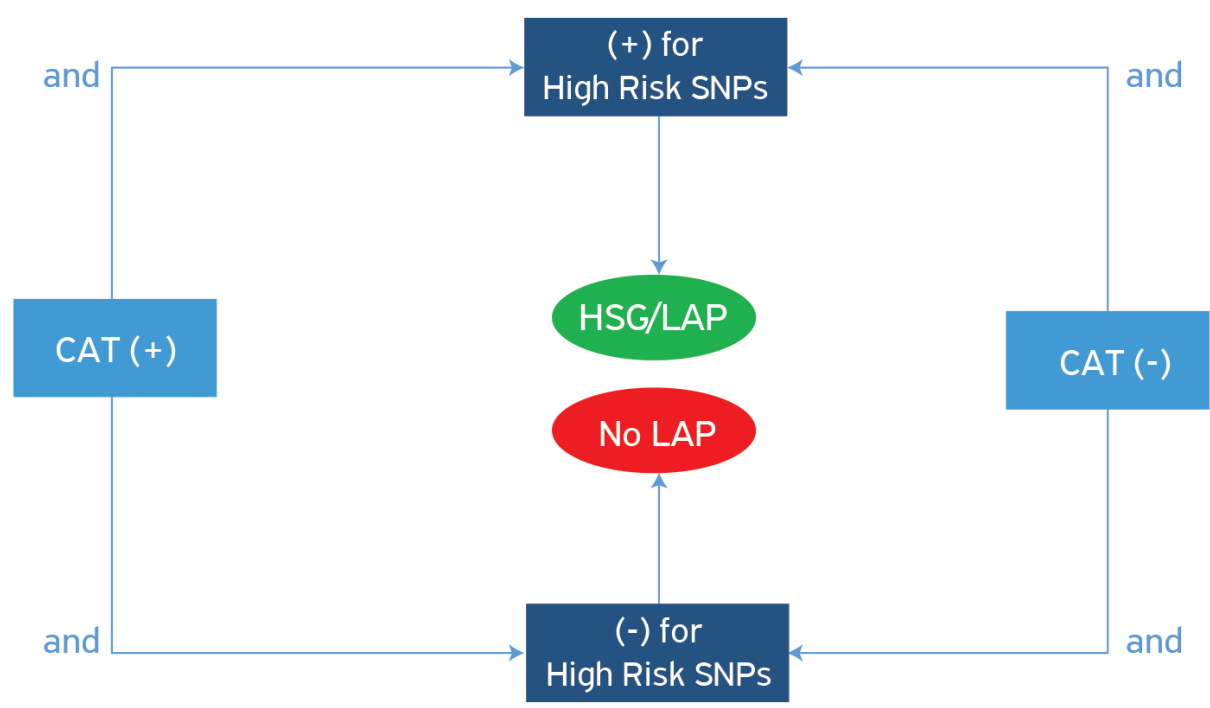

Figure 1. Elements of the decision making process based on the implementation of the "CAT + genome-based companion diagnostic" in the initial phases of the infertility screening strategy

For improving the prediction of risk for TFI, further immunogenetic studies need to focus on identifying and precisely combining SNPs into genetic traits. SNPS linked with a positive or negative effect on tubal pathology need to be combined in a way to provide an accurate assessment of ratio between risk factors and protective traits.

In addition to better predict tubal pathology, genetic markers could also improve the timing of infertility investigation. The timing of infertility investigation and treatment are crucial factors for avoiding both over and under treatment and providing an optimal management of infertility [42]. One of the advantages of the genome-based companion diagnostic is non-invasiveness which could justify earlier investigation of tubal pathology. The potential of genetic markers to improve the timing of infertility investigation, as well as timing of starting an adequate treatment should be explored. Women undergoing fertility investigation in the Netherlands are growing older (Chapter 4) making the timing of the fertility work up even more of an issue. 
Further, European surveillance and screening data on Chlamydia infection among young adults are showing a trend of persisting or increasing prevalence. At the same time, the trend of postponing the first pregnancy to the late 30 s has been noticed, leaving a long period of time for Chlamydia induced tubal pathology to be developed and not diagnosed. The future of TFI screening should preferably be studied considering the influence of these trends on the future demand for fertility care.

Then, the resources consumed by the new diagnostic strategy need to be weighed against the benefits of performing the test and the effects of redirecting these resources away from existing strategies. Genetic testing may undeservedly be labeled as high-priced. Recent study reviewing the entire spectrum of economic evaluations associated with genetic testing used for guidance treatments and interventions, found no evidence that genetic testing is either inferior or superior in terms of cost-effectiveness to other medical interventions. They found that how the genetic test is used, rather than whether it was used, had the economic significance [43]. Further research about economic implications of the introduction of genomic markers in the routine fertility work up is needed.

As discussed earlier, advances in the research of Chlamydia trachomatis host genetic factors could have significant implications for gynecology practice. In Chapter 5 we explored the potential barriers and facilitating factors for the introduction of genetic testing in screening for Chlamydia induced TFI, perceived by the Dutch gynaecologists. Clinicians' knowledge and attitudes regarding genetic tests influences the ability of genomics to have an impact on clinical practice. $77 \%$ of participants gave a positive response answering the question about the potential of genetic markers to increase the sensitivity of the diagnosis of tubal pathology. Cost-effectiveness was indicated by $100 \%$ of participants as an important factor in gaining their support for the new screening strategy regardless of their position at the department or the hospital they work in. Clinical utility is recognized as the most import indicator of the quality of a genetic test, with $56 \%$ of the respondents fully agreeing on it. Clinical utility is also considered somewhat more important than clinical validity. Earlier research regarding physician attitudes toward genetic testing for cancer susceptibility also identified cost-effectiveness and uncertainty about the accuracy, interpretation, and clinical utility of genetic tests for cancer as the main concern [44]. Results 
study also demonstrated the awareness of respondents of the fact that genomic advances are changing clinical management of diseases and the role of physicians in it. The majority of respondents expressed preferences for an additional training in clinical genetics prior to the introduction of the test. Earlier study on the promise of genomic research identified education and training for health-care professionals, designed to enable them to interpret more complex genomic data, as very important facilitating factors in translation of innovative genomic tests into clinical practice [45].

Evaluation of the potential of TFI screening based on host genetic determinants to meet the need of women undergoing fertility work up for a simple, accurate and noninvasive screening tool and exploration of the attitudes of fertility care doctors towards the use of genetic testing in the routine fertility work up, established a first step into a pre-implementation research. Preferably it should be followed by comprehensive cost-effectiveness evaluations of existing and proposed TFI screening strategies and by studies confirming improved health outcomes associated with the introduction of genetic testing into fertility work up. 


\section{REFERENCES}

1. CDC. Public Health Genomics. Available from: http://www.cdc.gov/ genomics/about/AAG/index.htm.

2. Roundtable on Translating Genomic-Based Research for Health. The Value of Genetic and Genomic Technologies: Workshop Summary. 2010, National Academies Press. Available from: www.ncbi.nlm.nih. gov/books/NBK52756.

3. WHO. Fact sheet on HIV/AIDS. Available from: http://www.who.int/ features/factfiles/hiv/en/.

4. Telenti, A and Johnson, WE. Host Genes Important to HIV Replication and Evolution. Cold Spring Harbor Perspectives in Medicine, 2012. 2(4).

5. Tang, $J$ and Kaslow, RA. The impact of host genetics on HIV infection and disease progression in the era of highly active antiretroviral therapy. AIDS, 2003. 17 Suppl 4: p. S51-60.

6. He, W, Castiblanco, J, Walter, EA, et al. Mendelian randomization: potential use of genetics to enable causal inferences regarding HIVassociated biomarkers and outcomes. Current Opinon in HIV and AIDS, 2010. 5(6): p. 545-559.

7. An, P and Winkler, CA. Host genes associated with HIV/AIDS: advances in gene discovery. Trends in Genetics, 2010. 26(3): p. 119-131.

8. O'Brien, SJ and Nelson, GW. Human genes that limit AIDS. Nature Genetics, 2004. 36(6): p. 565-574.

9. Kaslow, RA, Dorak, T and Tang, JJ. Influence of host genetic variation on susceptibility to HIV type 1 infection. Journal of Infectious Diseases, 2005. 191 Suppl 1: p. S68-77.

10. Singh, $\mathrm{KK}$ and Spector, SA. Host genetic determinants of human immunodeficiency virus infection and disease progression in children. Pediatric Research, 2009. 65(5 Pt 2): p. 55R-63R.

11. Henrich, TJ and Kuritzkes, DR. HIV- 1 entry inhibitors: recent development and clinical use. Current Opinion in Virology, 2013. 3(1): p. 51-57.

12. Haqqani, AA and Tilton, JC. Entry inhibitors and their use in the treatment of HIV-1 infection. Antiviral Research, 2013. 98(2): p. 158-170.

13. Gulick, R. New ARV drugs. BMC Infectious Diseases, 2014. 14(Suppl 2): p. S2.

14. Ma, JD, Lee, KC and Kuo, GM. HLA-B*5701 testing to predict abacavir hypersensitivity. PLoS currents, 2010. 2: p. RRN1203.

15. Haaland, RE, Johnson, JA and Tang, J. Recent Advances in Research of HIV Infection: Implications of Viral and Host Genetics on Treatment and Prevention. Public Health Genomics, 2013. 16(1-2): p. 31-36.

16. Julg, B, Moodley, ES, Qi, Y, et al. Possession of HLA Class II DRB 1 * 1303 Associates with Reduced Viral Loads in Chronic HIV-1 Clade C and B Infection. Journal of Infectious Diseases, 2011 . 203(6): p. 803-809.

17. Alter, G, Heckerman, D, Schneidewind, A, et al. HIV-1 adaptation to NKcell-mediated immune pressure. Nature, 2011 . 476(7358): p. 96-100. 
18. Goulder, PJR and Watkins, DI. HIV and SIV CTL escape: implications for vaccine design. Nature Reviews Immunology, 2004. 4(8): p. 630-640.

19. Koff, WC, Russell, ND, Walport, M, et al. Accelerating the development of a safe and effective HIV vaccine: HIV vaccine case study for the Decade of Vaccines. Vaccine, 2013. 31, Supplement 2(0): p. B204-B208.

20. Shea, PR, Shianna, KV, Carrington, M, et al. Host genetics of HIV acquisition and viral control. Annual review of medicine, 2013. 64: p. 203-217.

21. Chen, D and Gyllensten, U. Systematic investigation of contribution of genetic variation in the HLA-DP region to cervical cancer susceptibility. Carcinogenesis, 2014.

22. Hildesheim, A and Wang, SS. Host and viral genetics and risk of cervical cancer: a review. Virus Research, 2002. 89(2): p. 229-240.

23. Chen, X, Jiang, J, Shen, $\mathrm{H}$, et al. Genetic susceptibility of cervical cancer. Journal of Biomedical Research, $2011.25(3)$ : p. 155-164.

24. Yang, N, Eijsink, JJ, Lendvai, A, et al. Methylation markers for CCNA1 and C130RF18 are strongly associated with high-grade cervical intraepithelial neoplasia and cervical cancer in cervical scrapings. Cancer Epidemiology, Biomarkers \& Prevention, 2009. 18(11): p. 3000-3007.

25. Verhoef, VMJ, Heideman, DAM, van Kemenade, FJ, et al. Methylation marker analysis and HPV 16/18 genotyping in high-risk HPV positive self-sampled specimens to identify women with high grade CIN or cervical cancer. Gynecologic Oncology, (0).

26. Litjens, RJ, Theelen, W, van de Pas, Y, et al. Use of the HPV MLPA assay in cervical cytology for the prediction of high grade lesions. Journal of medical virology, 2013. 85(8): p. 1386-1393.

27. Bailey, RL, Natividad-Sancho, A, Fowler, A, et al. Host genetic contribution to the cellular immune response to Chlamydia trachomatis: Heritability estimate from a Gambian twin study. Drugs of today, 2009. 45 Suppl B: p. 45-50.

28. Meylan, E, Tschopp, J and Karin, M. Intracellular pattern recognition receptors in the host response. Nature, 2006. 442(7098): p. 39-44.

29. Darville, T, O’Neill, JM, Andrews, CW, Jr., et al. Toll-like receptor-2, but not Toll-like receptor-4, is essential for development of oviduct pathology in chlamydial genital tract infection. Journal of Immunology, 2003. 171(11): p. 6187-6197.

30. den Hartog, JE, Lyons, JM, Ouburg, S, et al. TLR4 in Chlamydia trachomatis infections: knockout mice, STD patients and women with tubal factor subfertility. Drugs of today, 2009. 45 Suppl B: p. 75-82.

31. Karimi, O, Ouburg, S, de Vries, HJ, et al. TLR2 haplotypes in the susceptibility to and severity of Chlamydia trachomatis infections in Dutch women. Drugs of today, 2009. 45 Suppl B: p. 67-74.

32. Morré, SA, Karimi, $\mathrm{O}$ and Ouburg, S. Chlamydia trachomatis: identification of susceptibility markers for ocular and sexually transmitted infection by immunogenetics. Fems Immunology And Medical Microbiology, 2009. 55(2): p. $140-153$. 
33. Brankovic, I, Ouburg, S, Spargaaren, J, et al. NOD1 functional polymorphism protects against Chlamydia trachomatis infection and increases the risk of tubal factor infertility: translation of immunogenetic factors into clinical setting. 2014. Unpublished paper.

35. A genome wide association scan reveals pathway-wide genomic differences between cases of scarring trachoma and controls. in The Thirteenth International Symposium on Human Chlamydial Infections2014: Pacific Grove, California, USA.

35. Roberts, CH, Franklin, C, Molina-Gonzales, $\mathrm{S}$ et al. A genome wide association scan reveals pathway-wide genomic differences between cases of scarring trachoma and controls. The Thirteenth International Symposium on Human Chlamydial Infections, Pacific Grove, California, USA, 22th-27th June 2014. Unpublished conference paper.

36. De Paoli, P. Bio-banking in microbiology: from sample collection to epidemiology, diagnosis and research. FEMS microbiology reviews, 2005. 29(5): p. 897-910.

37. Morré, SA, Ouburg, S, Pena, AS, et al. The EU FP6 EpiGenChlamydia Consortium: contribution of molecular epidemiology and host-pathogen genomics to understanding Chlamydia trachomatis-related disease. Drugs of today, 2009. 45 Suppl B: p. 7-13.

38. Haggerty, CL, Gottlieb, SL, Taylor, BD, et al. Risk of sequelae after Chlamydia trachomatis genital infection in women. The Journal of infectious diseases, 2010. 201 Suppl 2: p. S134-155.

39. Morré, SA, van den Brule, AJ, Rozendaal, L, et al. The natural course of asymptomatic Chlamydia trachomatis infections: $45 \%$ clearance and no development of clinical PID after one-year follow-up. International Journal of STD \& AIDS, 2002. 13 Suppl 2: p. 12-18.

40. Land, JA, Evers, JL and Goossens, VJ. How to use Chlamydia antibody testing in subfertility patients. Human reproduction, 1998. 13(4): p. 1094-1098.

41. Land, JA, Van Bergen, JE, Morré, SA, et al. Epidemiology of Chlamydia trachomatis infection in women and the cost-effectiveness of screening. Human Reproduction Update, 2010. 16(2): p. 189-204.

42. Gnoth, C, Godehardt, E, Frank-Herrmann, P, et al. Definition and prevalence of subfertility and infertility. Human reproduction, 2005. 20(5): p. 11441147.

43. Hatz, MM, Schremser, $\mathrm{K}$ and Rogowski, W. Is Individualized Medicine More Cost-Effective? A Systematic Review. PharmacoEconomics, 2014. 32(5): p. 443-455.

44. Freedman, AN, Wideroff, L, Olson, L, et al. US physicians' attitudes toward genetic testing for cancer susceptibility. American Journal of Medical Genetics Part A, 2003. 120A(1): p. 63-71.

45. Simonds, NI, Khoury, MJ, Schully, SD, et al. Comparative Effectiveness Research in Cancer Genomics and Precision Medicine: Current Landscape and Future Prospects. Journal of the National Cancer Institute, 2013. 
CHAPTER 7

VALORISATION ADDENDUM 


\section{Valorisation}

At the core of the valorisation process is the notion of translation of knowledge into products and services. The topic of our research was the translation of genomic information in the diagnosis and treatment of infectious diseases and, in particular, the facilitation of this translation in the diagnosis of Chlamydia trachomatis induced tubal factor infertility (TFI). Therefore, parts of our research were specifically addressing the concept of valorisation. We proposed a way of translating the knowledge generated through host immunogenetic research of Chlamydia trachomatis infection into routine fertility care and explored potential facilitators and barriers to the process of creating a healthcare service from such knowledge.

Advances in genomics reshaped our understanding of pathogenesis of infectious diseases, highlighted the role of host genetic determinants in modulating immune response and showed us that the differences in clinical course and outcome of infection between individuals can be attributed to their genetic differences, among other factors.

In the case of the intracellular bacteria Chlamydia trachomatis, host genetic research showed that the differences in the course and outcome of the infection can be explained by a patient's genetic makeup. Persistent and ascending Chlamydia trachomatis urogenital infection is one of the most common causes of damage to the fallopian tubes and consequent tubal factor infertility (TFI). Almost half of the cases of TFI can be attributed to a previous Chlamydial infection.

In the Netherlands, Chlamydia IgG antibody test (CAT) and Hysterosalpingography (HSG) are the two most commonly used tests for the primary investigation of TFI, they are usually followed by laparoscopy as the reference diagnostic. A positive CAT result means that a woman had an infection at some point in the past, but the test is often positive in the absence of tubal pathology. Almost half of women will clear the infection without any consequences within 12 months. Thus, the main disadvantage of CAT is a relatively high number of false positive results for identifying women with TFI. In 40-50\% of women who are testing positive for CAT no tubal pathology is found by laparoscopy. HSG is either performed following CAT or as an independent test of tubal patency. Its main disadvantage is that it is a painful procedure and can be complicated by an ascending infection. 
As a part of valorisation process we explored the health needs of women undergoing fertility investigation as well as the needs of clinicians for an accurate screening tool. Through our research we recognized the potential of host genetic findings to improve the investigation of Chlamydia induced TFI. We proposed combining genomic markers and CAT in the routine fertility work up with the aim of improving the accuracy of TFI investigation by correlating host genetics with infection outcomes, therefore more accurately determining the risk of TFI and reducing the number of misdiagnosis. The clinical relevance of such a change would be an improved clinical decision making for gynaecologists, a more efficient use of resources, thus less expenses for the healthcare system, and reduced interventions for the patient.

The social relevance of our topic and our findings stems from the high public health relevance of the Chlamydia trachomatis urogenital infection, shown by high prevalence of the infection and the serious complications associated with it. Costs associated with diagnosis and treatment of the associated infertility (including costs caused by complications of unnecessary laparoscopy and HSG) poses a significant economic burden for healthcare systems and individuals. In fact, it has been established that infertility is an area where healthcare costs, in many countries, are born more often by individual, creating significant economic disparities and having a wide societal impact. Furthermore, infertility has a strong impact on the quality of life of the affected couples thus having a wide societal impact as well.

Data on the prevalence of Chlamydia trachomatis urogenital infection prevalence obtained through the Dutch National Institute for Public Health and Environment surveillance efforts showed that in the period from 2010-2012 10-13\% of people visiting the Dutch regional Sexually Transmitted Infection (STI) clinics tested positive for Chlamydia. A pilot nationwide screening project for Chlamydia infection, conducted in 2003 in the Netherlands, found a $2 \%$ overall prevalence of Chlamydia infection in women. Later follow up screening projects found no indication of the decrease of these positivity rates. Additionally, European surveillance data demonstrate that Chlamydia trachomatis is at the moment the most commonly diagnosed bacterial STI and still no decline in prevalence is found overall. Therefore we can assume that Chlamydia will continue to play an important role in the pathophysiology of TFI. 
Additionally, a strong relation has been established between women's age and the demand for fertility care. The demand for fertility care is increasing with women's age. Recently in the Netherlands, similarly to in other high income countries, there is a trend of postponing the first pregnancy. In 2012, 20\% of all births were to first time mothers older than 35 . We can assume that this trend will persist. At the same time Chlamydia infection show trend of either persistent or increasing prevalence rates of the infection among young adults. These trends might have full impact on demand for fertility care in 10-15 years when now young adults become women in their late 30 s deciding to become mothers.

Genetic testing already has an important diagnostic/screening role in in the field of gynaecology. Prenatal genetic screening, essential part of new born screening, was in fact the first established program of population-wide genetic testing, and it has been a great public health success. Testing of chromosomal abnormalities and single gene disorders, such as such as cystic fibrosis and sickle cell anaemia traditionally done by invasive techniques, such as amniocentesis and chorionic villi sampling, was recently replaced by non-invasive genetic testing of free foetal DNA and free foetal cells in the mother's blood.

Additionally, genetic testing for cancer susceptibility to breast and ovarian cancer is often first discussed in the gynaecologist's office. Gynaecologists are advising patients of the possibility and necessity of that the appropriate cancer risk assessment and they are responsible for providing appropriate information about the test and consequent treatment options. However, genetic information has not been used so far in fertility investigation. Using patient's genomic information in routine fertility work up represents a novel approach in fertility care.

The primary audience for our research are clinicians. Advancements in genomics are driving change in diagnostic and treatment strategies, therefore clinicians need to repeatedly adjust to these changes. They are always in need of a good screening test, accurate in assessing the risk of TFI in patients and at the same time simple and inexpensive. Combining CAT testing and genetic test would affect the clinicians' decision making on subsequent investigations and consequent treatment. We believe, it could help avoiding unnecessary laparoscopies, expediting further investigation and use of Assisted Reproductive Technology 
(ART) when needed. Additionally, we think that patients' organization and health policy makers, scanning the horizon for more accurate and less invasive TFI screening strategies with the potential to improve fertility care, might be our audience too. Finally, our target group are also other researchers, especially those with in-depth knowledge about economic evaluations, who would build on our research by determining the efficiency of a new proposed TFI investigation strategy compared to the existing ones.

We also presented the results of our survey-based research about the attitudes of Dutch gynaecologists towards the addition of genetic biomarkers in the investigation of Chlamydia induced infertility. The results of this research provide a good foundation for the planning of further steps in the implementation of genetic testing into routine fertility work up. The first step in the further process of valorisation would be developing a high quality genetic test by combining different genetic marker traits in a way that provides an accurate assessment of risk factors and protective traits. Some patients may be positive for SNPS which increase the risk to develop TFI and at the same time be positive for SNPs of preventive genetic trait, making clinicians' decision process more complex and calling for precise algorithms to aid decision making. The combined genetic and CAT test will need to be optimized in a way which makes the grey zone of decision making as small as possible. Additionally, it is important that test has straightforward results, easy to interpret and explain to patients.

Finally, crucial steps in the implementation process would be establishing costeffectiveness and clinical utility of the test. Insufficient evidence on benefits and harms of genomic tests in clinical setting has been identified as a translational barrier. Clinicians involved in our survey put the emphasis on the importance of clinical utility. Proven clinical utility of the test means the ability of the test to lead to improved health outcomes. In the case when test is used for disease screening and/or risk assessment, clinical utility is defined as improved health outcomes based on provision of information useful for personal or clinical decision making and better patient management and/or improved health outcomes based on prevention or early detection strategies. 
Furthermore, in our survey about the clinicians' attitudes towards the introduction of genetic testing in the routine screening for TFI cost-effectiveness was indicated by all of the participants, regardless of their position at the department or the hospital they work, as an important factor in gaining their support. Cost-effectiveness analysis is the most commonly used form of full economic evaluation, in this analysis costs and consequences of two or more alternatives are being compared. Cost-effectiveness analysis can be performed from different perspectives. The most appropriate in this case would be to perform the analysis from the societal perspective, thus taking into consideration all the relevant costs.

In summary, the research in my thesis has shown great potential for valorisation since increase in accuracy of TFI screening could significantly improve the quality of fertility care. 
SUMMARY 

Advances in genomics created high expectations regarding the impact that those discoveries would have on healthcare. This process of responsible, effective and timely translation of genome-based information and technologies into health policies and practice for the benefit of population health is the core task of the field of Public Health Genomics. The focus of this thesis is the effective and timely translation of genome-based information in the field of infectious diseases.

General Introduction provides an introduction of the topic and the outline of the thesis. The aim of this thesis was to explore the translation of genomic information in the diagnosis and treatment of infectious diseases in general and to improve and facilitate this translation in the diagnosis of Chlamydia induced tubal factor infertility (TFI) in particular. The objective of the Part I (Chapters 1 and 2 ) of the thesis was to provide an overview of the basic genomic and genetic findings with translational potential for application in diagnosis and treatment of Sexually Transmitted infections (STIS) of a high public health relevance and to explore the role of biobanks in research of infectious disease host genomics. The objective of the Part II (Chapters 3-5) of the thesis was to assess health needs of the Dutch women seeking fertility care, to explore strategies for the introduction of genomic biomarkers into the diagnosis of tubal infertility and to evaluate the attitudes of the Dutch gynecologist toward the implementation of the genomic biomarkers into routine infertility diagnosis.

\section{Part I}

The Human Genome Project opened the door for the translation of genome-based knowledge into every day clinical care. In Chapter $\mathbf{1}$ the translational potential of basic genomic and genetic findings for HIV, CT, and HPV for application in public health and in diagnostics, treatment, and prevention of late complications of these infectious diseases was reviewed. We found many host genetic variants having a role in modulating the immune response to HIV, HPV and Chlamydia infections in the literature. Nevertheless, we also noticed an imbalance between the number of confirmed gene-disease associations and the number of practical applications of that knowledge.

In Chapter $\mathbf{2}$ we examined the role of biobanks in basic research of infectious disease genomics, as well as their relevance in the translation of impending 
knowledge and in the clinical uptake of genome-based knowledge in infectious diseases. We described the trend of growing numbers of biobanks which are founded and governed by hospitals and academic institutions and support the research in infectious disease genomics. We explored successful examples of research deriving from these biobanks. We also noticed that the trend of development of nation-wide or transnational collections of human and microbial samples and building large consortia and networks is ever increasing.

\section{Part II}

In Chapter 3 an overview of the current state of the art of host genetic markers related to Chlamydia trachomatis infection is provided. By combining multiple SNPs in the Pathogen Recognizing Receptor (PRR) genes and genes in linked pathways, and combining them as susceptibility markers, a highly predictive test for tubal pathology-based subfertility can potentially be developed. We investigated the potential translational and clinical value of adding these diagnostic host genetic marker profiles to the current clinical management of infertility. A public health genomic (PHG) model as a possible facilitator in the process of implementing host genetic markers into clinical applications is considered.

In order to facilitate the translation process of Chlamydia trachomatis related host genomic markers influencing the course and outcome infection, in Chapter 4 we explored the potential need for introducing genomic testing in the routine investigation of tubal factor infertility (TFI) by assessing health needs of the target population. We designed a 3-step framework for the assessment of fertility care-related health needs. In step 1, we identified services/strategies currently in place in fertility care in the Netherlands and found no consensus on the sequence of diagnostic procedures in routine fertility work-up, including investigation of tubal pathology as a possible cause. In step 2, we defined the characteristics of the target population for fertility care in the Netherlands and assessed their health needs. The population of women undergoing fertility investigation in the Netherlands is growing older and their demand for fertility care greater. Overall, the data show 5-10\% annual increase in demand for medical assisted reproduction in many developed countries over the last 5 years. Screening and surveillance data on Chlamydia infection show either persistent or increasing prevalence rates of the infection among young adults. This might 
have clinical implications in 10 to 15 years, resulting in increased number of women being assessed for the risk of TFI. Finally, in step 3 we proposed a strategy for improving the accuracy of the first line testing by correlating host genetics with infection outcomes. Our new proposed screening strategy would aim to more accurately determine the risk of TFI and to reduce the number of misdiagnosis through synergistic action of serology and genetic testing, by taking blood samples for testing Chlamydia serology (CAT) and host genomic biomarkers (SNPs) early in fertility work-up.

In Chapter $\mathbf{5}$ we assessed Dutch gynaecologists' attitudes towards the introduction of genetic testing in the routine screening for TFI and evaluated their knowledge about the genetic background behind different courses and outcomes of Chlamydia infection. We developed a questionnaire for the doctors working at the OB/GYN departments about their attitudes towards the addition of genetic testing in the diagnosis of TFI. Using the questionnaire, we surveyed 48 doctors providing fertility care in Academic hospitals in Maastricht, Groningen and Utrecht, in order to investigate what they perceive to be major challenges and major facilitating factors in introducing genetic testing in routine fertility work up. Cost-effectiveness was indicated by $100 \%$ of participants as an important factor in gaining their support for the introduction of the new screening strategy regardless of their position ate the department or the hospital they work in. Clinical utility is recognized as the most import indicator of the quality of a genetic test with $56 \%$ of the respondents fully agreeing that clinical utility is the most important indicator.

In Chapter 6 we discussed the findings described in this thesis and offered some recommendations for future studies. 
De vooruitgang geboekt in genomics heeft hoge verwachtingen geschept wat betreft de invloed die deze ontwikkelingen hebben op de gezondheidszorg. Het proces van verantwoorde, effectieve en tijdige translatie van informatie en technologieën gebaseerd op het genoom naar beleid en beoefening in de gezondheidszorg ter bevordering van de volksgezondheid is de kerntaak van het veld Public Health Genomics. Dit proefschrift focust op de effectieve translatie van genoomgebaseerde informatie in relatie tot infectieziekten.

De General Introduction geeft een algemeen overzicht en beschrijft de achtergrond van translatie van genoom-gebaseerde informatie gerelateerd aan infectieziekten, daarna volgt in dit eerste hoofdstuk een uiteenzetting van de onderwerpen in dit proefschrift. Het doel van dit proefschrift was het exploreren van de translatie van genoominformatie naar de diagnose en behandeling van infectieziekten in het algemeen en in het bijzonder om deze translatie te faciliteren en te verbeteren binnen de diagnose van Chlamydia-geïnduceerde tubal factor infertiliteit (TFI). Het doel van Part I (hoofdstukken 1 en 2) van dit proefschrift is om een overzicht te geven van bevindingen binnen het veld van de genetica met translationele potentie voor de toepassing in de diagnose en behandeling van seksueel overdraagbare aandoeningen (SOAs) met een hoge relevantie voor de volksgezondheid en om de rol te exploreren van biobanken in het onderzoek naar de host genomics bij infectieziekten. Het doel van Part II (hoofdstuk 3-5) van het proefschrift is om de gezondheidszorgbehoeften in te schatten van Nederlandse vrouwen die fertiliteitszorg zoeken, om strategieën te bepalen voor de introductie van genetische biomarkers in de diagnose van TFI en om de mening van Nederlandse gynaecologen is ten opzichte van de toepassing van genetische biomarkers in hun klinische routine te inventariseren.

\section{Part I}

Het Human Genome Project verschafte toegang tot de translatie van genoomgebaseerde kennis in de dagelijkse klinische zorg. Chapter I geeft een overzicht van de translationele mogelijkheden van genetische bevindingen voor HIV, CT, en HPV in volksgezondheid, diagnose, behandeling en preventie van late complicaties van deze infectieziekten. Wetenschappelijke literatuur beschrijft vele genetische varianten van de gastheer die een rol spelen in het modelleren van de immuunreactie tegen HIV, HPV en CT infecties. Desalniettemin blijkt er een disbalans te zijn tussen het aantal gene-disease associations en het aantal klinische toepassingen gerelateerd aan deze associaties. 
SAMENVATTING 

In Chapter $\mathbf{2}$ hebben we de rol van biobanken onderzocht aangaande het onderzoek naar genomics binnen infectieziekten, alsmede hun rol in de translatie van kennis en het toepassen van infectious disease genomics in de klinische praktijk. In dit hoofstuk wordt de trend beschreven van een groeiend aantal biobanken die opgericht en bestuurd worden door ziekenhuizen en academische instituten en die het onderzoek naar genomics in infectieziekten ondersteunen. We hebben succesvolle voorbeelden van onderzoek voortkomend uit informatie van deze biobanken, beschreven en daarnaast tevens een trend opgemerkt in de toenemende ontwikkeling van nationale of transnationale databases van humane en micro-organische samples en de opzet van grote consortia en netwerken.

\section{Part II}

Chapter 3 biedt een overzicht van de huidige stand van zaken met betrekking tot host genetic markers gerelateerd aan Chlamydia trachomatis infectie. Door het combineren van meerdere single nucleotide polymorfisms (SNPs) in de pathogen recognition receptor (PRR) genen en van genen in gerelateerde pathways, welke vervolgens als vatbaarheidsmarkers gecombineerd kunnen worden, kan mogelijk een hoog-voorspellende test voor TFI-geïnduceerde subfertiliteit ontwikkeld worden. We hebben onderzocht wat de potentiële translationele en klinische waarde van het toevoegen van deze diagnostische genetische markers uit het gastheerprofiel zou kunnen zijn voor het huidige klinische management van infertiliteit. Als een mogelijke faciliterende factor in het implementatieproces van host genetic markers in klinische toepassingen wordt een public health genomic (PHG) model beschreven.

Om de translatie van Chlamydia trachomatis gerelateerd aan host genetic markers te faciliteren, hebben we in Chapter $\mathbf{4}$ de mogelijke behoefte om genoomtesten in de triage van TFI te introduceren verkend door de gezondheidszorgbehoeften in de doelpopulatie te bestuderen. We hebben een 3-staps kader ontworpen om deze inschatting te kunnen maken. In de eerste stap hebben we services/ strategieën geïdentificeerd die momenteel in de fertiliteitszorg in Nederland gebruikt worden. Daarbij hebben we geen consensus gevonden over de volgorde van diagnostische handelingen in de klinische routine ook niet aangaande onderzoek naar ovariumpathologie als mogelijke oorzaak. In stap 2 hebben we de karakteristieken gedefinieerd van de doelpopulatie voor fertilteitszorg in Nederland en is de gezondheidszorgbehoeften van deze populatie bestudeerd. 
De gemiddelde leeftijd van de populatie vrouwen die fertiliteitsonderzoek ondergaat neemt toe evenals de vraag vanuit deze populatie naar fertiliteitszorg. Gedurende de afgelopen 5 jaar is in een groot aantal Westerse landen een jaarlijkse toename gevonden van $5-10 \%$ in de vraag naar medische interventie bij de reproductie. Screening en surveillance data van Chlamydia-infecties beschrijven persistente of toenemende prevalentie aantallen aangaande de infecties onder jongvolwassenen. Dit heeft mogelijk klinische implicaties over 10 tot 15 jaar, resulterend in een toenemend aantal vrouwen die onderzocht zullen worden op mogelijke TFI. Ten slotte, in stap 3 hebben we een strategie voorgesteld om de nauwkeurigheid van de eerstelijnstest te verbeteren door host genetic markers te combineren met de huidige anamnese. Onze voorgestelde screeningsmethode richt zich op een meer nauwkeurig geïnventariseerd risico op TFI en om het aantal misdiagnoses te verkleinen door synergistische aanpak van serologie en genetisch testen door middel van het nemen van bloedmonsters vroeg in het onderzoek naar fertiliteit en te testen op Chlamydia serologie (CAT) en host genetic markers (SNPs).

In Chapter $\mathbf{5}$ hebben we de houding van Nederlandse gynaecologen onderzocht met betrekking tot het introduceren van genetisch testen in de routine screening voor TFI en we hebben hun kennis geëvalueerd aangaande de genetische achtergrond van verschillend verloop en uitkomsten van Chlamydia infecties. We hebben een vragenlijst ontwikkeld voor artsen die bij OB/GYN-afdelingen werkzaam zijn betreffende hun attitude jegens het toevoegen van genetisch testen in de diagnose van TFI. Gebruik makend van deze vragenlijst hebben we 48 artsen bevraagd die fertiliteitszorg voorzien in academische ziekenhuizen in Maastricht, Groningen en Utrecht, met als doel om te onderzoeken wat zij als belangrijke uitdagingen en faciliterende factoren zien in het introduceren van genetisch testen in de routinezorg voor fertiliteit. Kost-effectiviteit werd door $100 \%$ van de deelnemers als een belangrijke factor aangegeven voor hun steun voor de introductie van de nieuwe strategie, onafhankelijk van hun positie binnen de afdeling of het ziekenhuis waar zijn werkzaam zijn. Klinische utiliteit werd door $56 \%$ van de respondenten aangegeven als de meest belangrijke indicator van de kwaliteit van een genetische test.

In Chapter 6 worden de bevindingen die in deze thesis zijn beschreven nogmaals uiteengezet en worden een aantal aanbevelingen gedaan voor toekomstig onderzoek. 



\section{Acknowledgments}

Foremost I would like to express my sincere gratitude to my supervisors. I would especially like to thank Prof. Dr. Servaas Morré for his enthusiasm, his immense knowledge and experience. It would not have been possible to write this doctoral thesis without his guidance in the time of research and his feedback in writing of the thesis. When I moved across the ocean, he still managed to answer my urgent e-mails that I would send forgetting about the time difference. Servaas, thank you for your kindness and your continuous help. I am very grateful to Prof. Dr. Angela Brand for the opportunity I have had to work and do research at the Maastricht University. Angela, I have enjoyed the opportunity to watch and learn from your knowledge and experience.

I would have never been able to finish my dissertation without support from my co-supervisor and my friend Dr. Elena Ambrosino. I am very grateful to you for your help in polishing my scientific arguments, for your continuous encouragement and your talent to cheer me up in moments of doubt.

I would like to thank Prof. Dr. Hans Maarse, my mentor for the Master thesis for helping me in overcoming my fear of writing and convincing me I could even be quite good at it.

I would like to thank Dr. Sander Ouburg for more than once coming to the rescue at the crucial moment.

I am thankful to Marleen Jansen and Dr. Joep van Berkel for the translation of the thesis summary to Dutch.

I would also like to thank my colleagues and co-authors with whom I have enjoyed many useful and entertaining discussions.

Last but not least, I am very thankful to my friend Mima Petković for her help with the thesis layout and preparation for the printing. 



\section{Curriculum Vitae}

Jelena Malogajski graduated from the Medical School of Belgrade University in Serbia in 2004. After one year of internship at the Clinical Center of Serbia she obtained the national medical license and worked at a small outpatient clinic in Belgrade, providing primary care, preventive care and health education. From 2006 till 2009 she worked at the National Institute of Public Health of Serbia. She worked at the Center for Control and Prevention of Non Communicable Diseases where she was organizing and managing data collection of diabetes incidence and mortality in Serbia, collaborating with and coordinating 22 regional public health centers. She was also a member of the team specialized in Health Analysis and Planning of the Healthcare System. She was involved in planning of health services based on service availability and usage and was responsible for the analysis and planning of primary care provided to newborns and preschool children in Serbia. In 2008, as a recipient of the Scholarship for Public Health professionals from the Dutch Ministry of Foreign Affairs she did the Master in Health Policy, Economics and Management at the Faculty of Health, Medicine and Life Sciences, at the Maastricht University in the Netherlands. In 2009, she started her PhD in the field of Public Health genomics of infectious diseases at the Institute for Public Health Genomics at the Maastricht University. In 2013 she moved to New York where she finished writing her thesis. 

APPENDIX 
Attitude ten opzichte van de toevoeging de diagnose van subfertiliteit ten gevolge van een Chlamydia trachomatis infectie

\section{Deel I: Algemene gegevens}

In deze vragenlijst staat de diagnosemethode van subfertiliteit als gevolg van een infectie met Chlamydia trachomatis centraal. Binnen de context van deze infectie, proberen wij te achterhalen of het volgens artsen en specialisten waardevol zou zijn om een genetische test toe te voegen. Over de genetische test volgt later meer informatie, we beginnen deze vragenlijst namelijk met een aantal algemene gegevens en een inventarisatie van de huidige stand van zaken, vanuit uw oogpunt. Wat is uw huidige positie?

Co-assistent

AIOS

ANIOS

Gynaecoloog

Anders n.l.,

1. Kunt u hieronder aangeven binnen welke leeftijdsgroep u valt?
$\square<25$ jaar
$25-30$ jaar
$35-40$ jaar
$40-45$ jaar
$\square \quad 45-50$ jaar
$50-55$ jaar
$\square \quad 30-35$ jaar
$55-60$ jaar
$\geq 60$ jaar

2. Wat is uw geslacht?
Man
Vrouw

3. Wat is de naam van het ziekenhuis waar u werkzaam bent?

4. Wat is de naam van de afdeling?

5. Hoeveel jaar ervaring heeft u binnen uw huidige positie? 
$0-5$ jaar

5-10 jaar

$10-20$ jaar

$\geq 20$ jaar

\section{Deel II: Genetica}

Chlamydia trachomatis kan als gevolg van de lichamelijke immuunrespons complicaties in de lagere delen van de tractus genitalis veroorzaken. Als de immuunrespons verstoord is, kan dit risico toenemen. Variaties in de immuunrespons zijn deels te verklaren door variaties in het genetisch profiel van een persoon. Genen die hierbij gerelateerd worden aan Chlamydia trachomatis complicaties zijn bijvoorbeeld genen die coderen voor receptoren voor pathogeen herkenning op immuuncellen. In kaart brengen van deze variaties zou gecombineerd met de Chlamydia Antilichaam Test (CAT) tijdens de diagnose van subfertiliteit mogelijk meer informatie kunnen bieden. Tijdens deze test worden de relevante variaties gefilterd tijdens de analyse en alleen die variaties bekeken die van belang zijn voor de diagnose.

1. Uit onderzoek is gebleken dat $40 \%$ van het verschil in het klinisch verloop van Chlamydia trachomatis tussen personen toegerekend kan worden aan genetische factoren van de patiënt.

Vindt u deze informatie bruikbaar voor diagnostiek van subfertiliteit die door Chlamydia trachomatis geïnduceerd is?

$\square \mathrm{Ja}$

$\square$ Nee

\section{Geen mening}

2. Denkt u dat het samenvoegen van genetische markers met een CAT de sensitiviteit van het diagnoseproces voor subfertiliteit zal veranderen?

$\mathrm{Ja}$, verlagen

Ja, verhogen

Nee

Geen mening 
3. Zou $u$ het introduceren van genetische testen in het onderzoek naar subfertiliteit steunen?

$\square \mathrm{Ja}$

$\square$ Nee

$\square$ Geen mening

4. Als deze test ingevoerd zou worden, waar vindt $u$ dat de voorgestelde combinatie van de twee testen plaats zou moeten vinden?

Bij de huisarts

Door de gynaecoloog in het ziekenhuis

Door de klinisch geneticus in het ziekenhuis

Anders, namelijk

\section{Deel III: CAT en genetische test in het diagnoseproces}

Geef alstublieft aan in hoeverre $u$ het eens bent met de volgende stellingen.

1. Het introduceren van genetische testen in het diagnoseproces van subfertiliteit zal de accuraatheid van de diagnose vergroten.

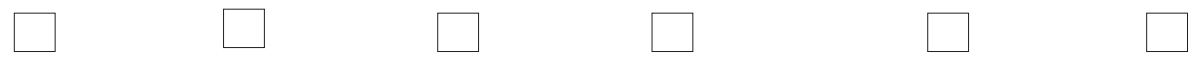

2. Het introduceren van genetische testen in het diagnoseproces van subfertiliteit zal het aantal fout positieve of negatieve diagnoses verkleinen.

Mee eens Deels mee eens Neutraal Deels mee oneens Mee oneens Geen mening

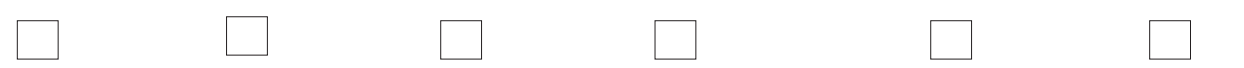

3. De gecombineerde test zal de tijd tot de diagnose van tubapathologie verlengen.

$\begin{array}{llll}\text { Mee eens } & \text { Deels mee eens } & \text { Neutraal Deels mee oneens } \\ \square\end{array}$

4. Genetische testen zijn te duur.

$\begin{array}{ccc}\text { Mee eens } & \text { Deels mee eens } \\ \square\end{array}$


5. Ik zou de introductie van combinatie van serologie en genetische test in de dagelijkse praktijk steunen als de test kosteneffectief is.

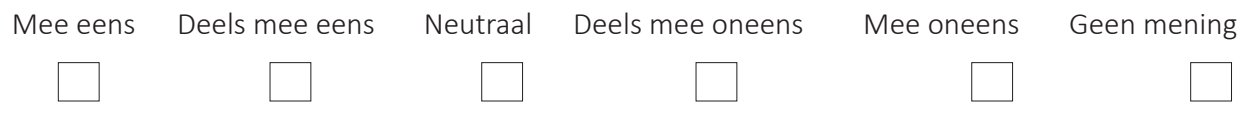

6. De belangrijkste indicatie van de kwaliteit van een $\mathrm{g}$ enetische test, is de mogelijkheid van de test om accuraat en betrouwbaar de relevante aandoening vast te stellen of te voorspellen (klinische validiteit)
Mee eens
Deels mee eens
Neutraal
Deels mee oneens
Mee oneens
Geen mening
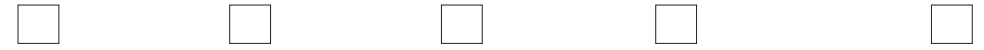

7. De belangrijkste indicatie van de kwaliteit van een genetische test, is een verbeterde gezondheidsuitkomst door de diagnose en daarop afgestemde behandeling (klinische utiliteit)

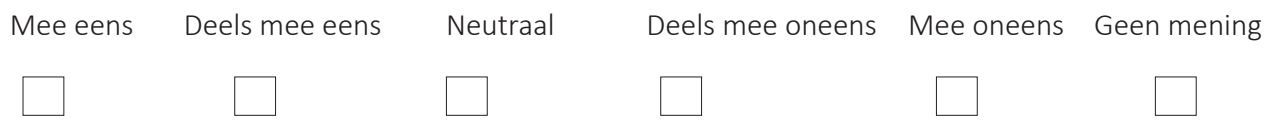

8. Ik zou de introductie van deze genetische test in de dagelijkse praktijk steunen mits de klinische validiteit en utiliteit aangetoond zijn.

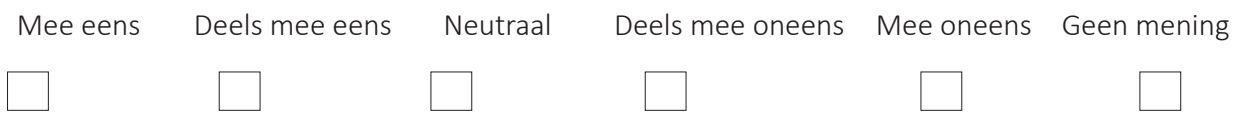

9. Mijn patiënten zouden de voorkeur geven aan niet-invasieve tests.

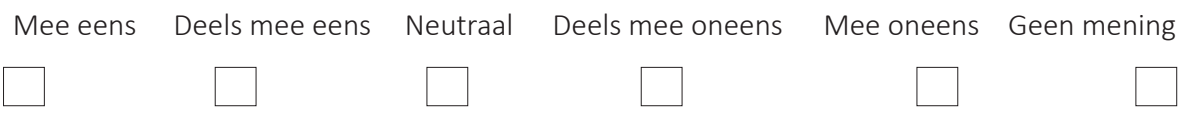


10. Mijn patiënten zouden de voorkeur geven aan een genetische test in plaats van een HSG.

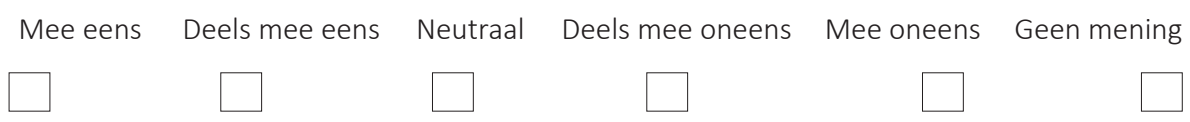

11. Mijn patiënten zouden de voorkeur geven aan een laparoscopie in plaats van een genetische test.

Mee eens Deels mee eens Neutraal Deels mee oneens Mee oneens Geen mening
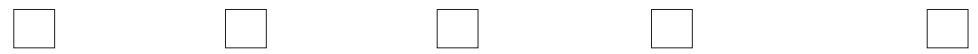

12. Ik zou de voorkeur van mijn patiënten wat betreft testen voor tubapathologie honoreren.

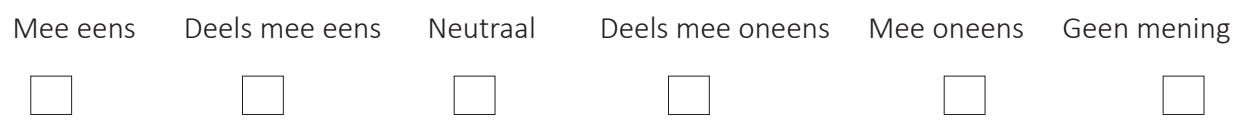

13. Als er een klinisch geneticus bij het diagnoseproces betrokken zou worden, zou ik het gevoel hebben dat de rol van de gynaecoloog binnen het diagnoseproces verkleind wordt.

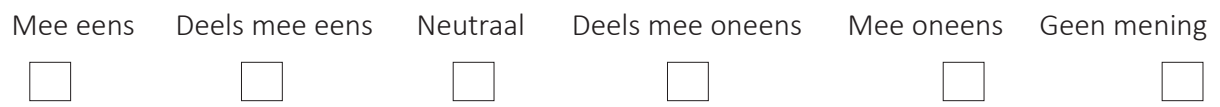

14. Ik zou een training nodig hebben als een genetische test aan het diagnoseproces van subfertiliteit zou worden toegevoegd.

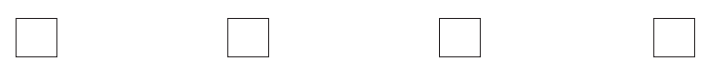


Heeft u nog overige opmerkingen of aanvullingen?

Hartelijk dank voor het invullen van deze vragenlijst.

Hartelijk dank voor uw deelname aan dit onderzoek. Als u graag meer informatie ontvangt over het onderzoek of nog vragen heeft over bijvoorbeeld deze vragenlijst, kunt u contact met ons opnemen. 
